\title{
AN IMPROVED PRECISE POINT POSITIONING MODEL USING GPS AND GALILEO OBSERVATIONS
}

by

\author{
Akram Afifi \\ B.Sc. (Hons), Cairo University, 2005 \\ M.Sc., Cairo University, 2009
}

A DISSERTATION

PRESENTED TO RYERSON UNIVERSITY

IN PARTIAL FULFILLMENT OF THE

REQUIREMENTS FOR THE DEGREE OF

DOCTOR OF PHILOSOPHY

IN THE PROGRAM OF

CIVIL ENGINEERING

Toronto, Ontario, Canada, 2015

(C) Akram Afifi 2015 


\section{DECLARATION}

I hereby declare that I am the sole author of this dissertation.

I authorize Ryerson University to lend this dissertation to other institutions or individuals for the purpose of scholarly research.

Akram Afifi

I further authorize Ryerson University to reproduce this dissertation by photocopying or by other means, in total or in part, at the request of other institutions or individuals for the purpose of scholarly research.

I understand that my dissertation may be made electronically available to the public.

Akram Afifi 


\title{
AN IMPROVED PRECISE POINT POSITIONING MODEL USING GPS AND GALILEO OBSERVATIONS
}

\author{
Doctor of Philosophy 2015 \\ Akram Afifi \\ Department of Civil Engineering \\ Ryerson University
}

\begin{abstract}
Precise point positioning (PPP) allows for centimeter- to decimeter-level positioning accuracy using a single global navigation satellite system (GNSS) receiver. However, the use of PPP is presently limited due to the time required for the solution to converge or re-converge to the expected accuracy, which typically requires about 30 minutes. This relatively long convergence time is essentially caused by the existing un-modeled GNSS residual errors. Additionally, in urban areas, the number of visible satellites is usually limited when a single satellite constellation is used, which in turn slows down the PPP solution convergence. This, however, can be overcome by combining the observations of two constellations, namely the GPS and Galileo systems.
\end{abstract}

Unfortunately, combining the GPS and Galileo constellations, although enhances the satellite geometry, introduces additional biases that must be considered in the observation mathematical models. These include the GPS-to-Galileo time offset, and Galileo satellite and receiver hardware delays. In addition, the stochastic characteristics of the new Galileo E1 and E5a signals must be determined to a high degree of precision. This can be done by analyzing various sets of GPS and Galileo measurements collected at two stations with short separation.

Several PPP models are developed in this dissertation, which combine GPS and Galileo observations in the un-differenced and between-satellite single-difference (BSSD) modes. These include the traditional un-differenced model, the decoupled clock model, the semi-decoupled clock model, and the between-satellite single-difference model. It is shown that the traditional 
un-differenced GPS/Galileo PPP model, the GPS decoupled clock model, and semi-decoupled clock GPS/Galileo PPP model improve the convergence time by about $25 \%$ in comparison with the un-differenced GPS-only PPP model. In addition, the semi-decoupled GPS/Galileo PPP model improves the solution precision by about $25 \%$ compared to the traditional un-differenced GPS/Galileo PPP model. Moreover, the BSSD GPS/Galileo PPP model improves the solution convergence time by about 50\%, in comparison with the un-differenced GPS PPP model, regardless of the type of BSSD combination used. As well, the BSSD model improves the solution precision by about $50 \%$ and $25 \%$ when the BSSD loose and tight combinations are used, respectively, in comparison with the un-differenced GPS-only model. 


\section{ACKNOWLEDGMENTS}

Above all, I am indebted to ALLAH who has given me the health, strength and patience, and has granted me the opportunity to complete my graduate studies at Ryerson University.

First, I would like to express my special thanks and gratitude to my distinguished supervisor, Prof. Ahmed El-Rabbany, for his professional supervision, encouragement, valuable suggestions, guidance, and proposed ideas throughout my study and this research. His abundant cooperation and understanding deserve unbounded appreciation. It was my great pleasure to work under his supervision.

In addition, I wish to extend my appreciation and thanks to members of the Ph.D. examining committee, Prof. Peter Dare, Prof. Songnian Li, Dr. Ahmed Shaker, and Dr. John Enright, for dedicating their valuable times to review my thesis.

I deeply appreciate the financial support from Ryerson University and the Government of Ontario, including the ATOP Award, Ryerson Graduate Award, Ryerson International Student Scholarship, and the Ontario Graduate Scholarship (OGS). In addition, indirect financial support was provided through the student competitions organized by the Association of Ontario Land Surveyors (AOLS).

Appreciations are given to the Geodetic survey division of Natural Resources Canada (NRCan) for providing the source code of the GPSPace PPP software. In addition, Dr. Mohamed Elsobeiey is acknowledged for providing the GPS-related modifications to GPSpace. The author is also grateful to Mr. Paul Collins from NRCan for providing the GPS decoupled clock products.

I would like to express a special thanks to my dear friend Dr. Wai Yeung Yan for his valuable support and precious ideas through my graduate studies. Finally, many thanks go to all of my colleagues in the Department of Civil Engineering for the unforgettable moments, friendship, and the support I received throughout the entire course of my research. 


\section{DEDICATION}

To the people who praise nothing but noble values and deserve vast respectMy parents, my wife, and my new baby 


\section{TABLE OF CONTENTS}

DECLARATION ii

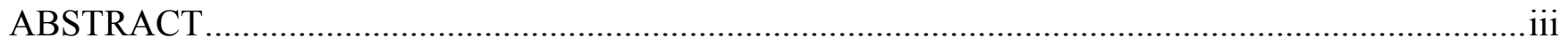

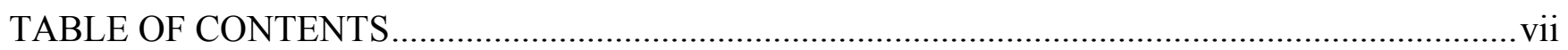

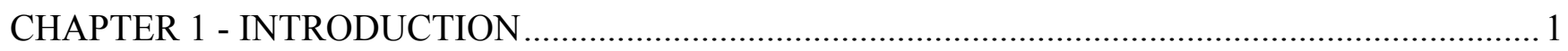

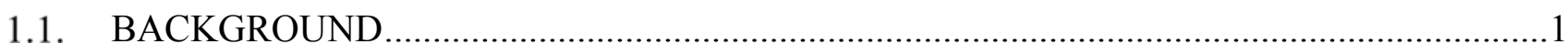

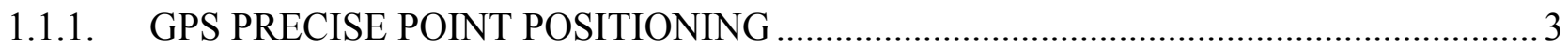

1.1.2. STOCHASTIC MODELING OF THE GNSS SIGNALS_..............................................

1.1.3. GPS/GALILEO PRECISE POINT POSITIONING ..................................................... 6

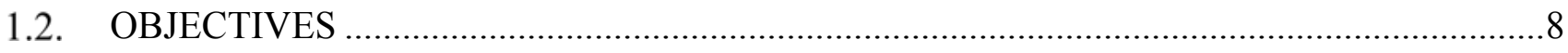

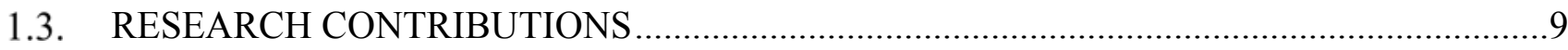

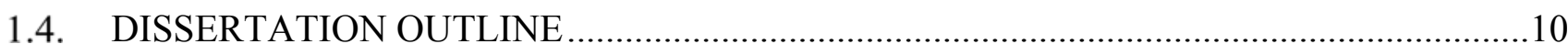

CHAPTER 2 - SINGLE FREQUENCY GPS/GALILEO PRECISE POINT POSITIONING USING UN-DIFFERENCED AND BETWEEN SATELLITE SINGLE DIFFERENCE MEASUREMENTS . 13

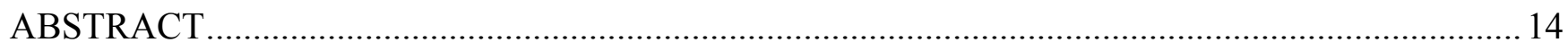

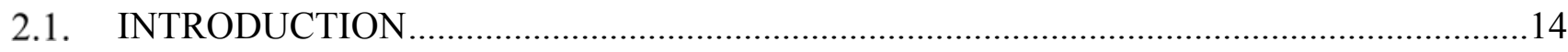

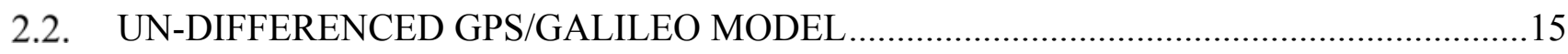

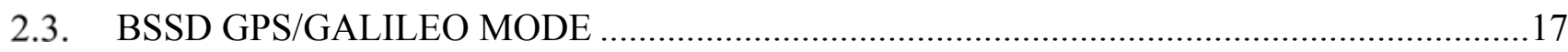

2.4. SEQUENTIAL LEAST SQUARES ESTIMATION …………...........................................

2.5. STOCHASTIC MODEL DEVELOPMENT ………………………………………….......

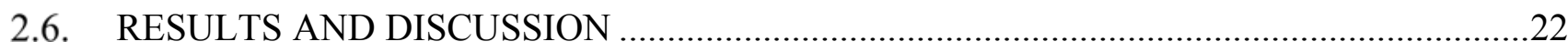

2.6.1. UN-DIFFERENCED POSITIONING RESULTS......................................................... 


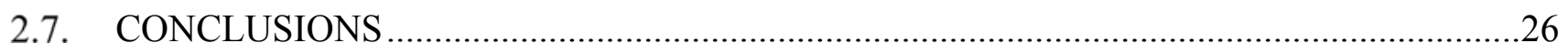

CHAPTER 3 - AN IMPROVED MODEL FOR SINGLE FREQUENCY GPS/GALILEO PRECISE POINT POSITIONING

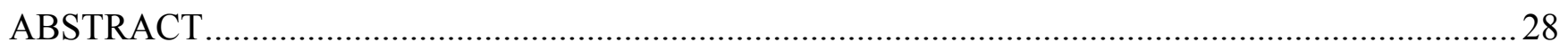

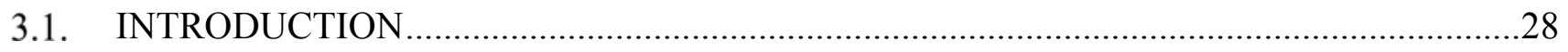

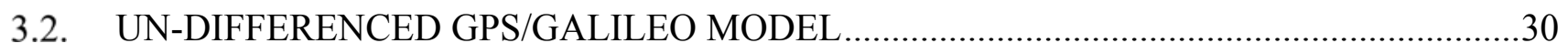

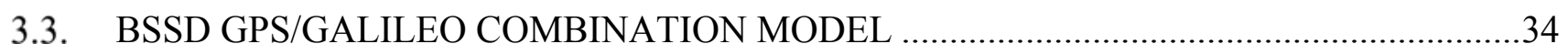

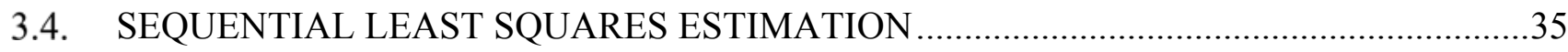

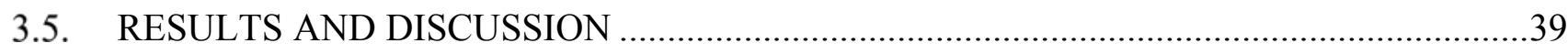

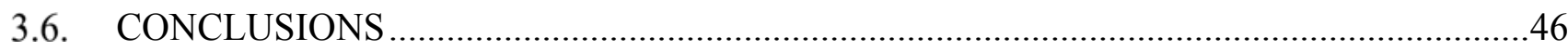

CHAPTER 4 - AN IMPROVED BETWEEN-SATELLITE SINGLE-DIFFERENCE PRECISE POINT POSITIONING MODEL FOR COMBINED GPS/GALILEO OBSERVATIONS.............................47

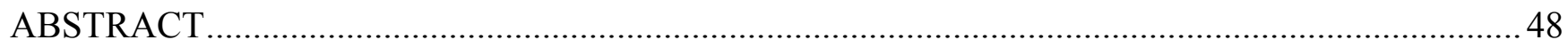

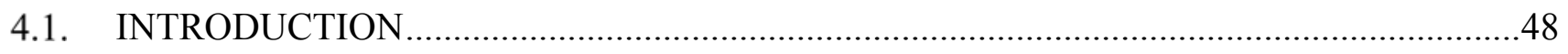

4.2. GPS/GALILEO PPP MATHEMATICAL MODEL .........................................................50

4.3. LEAST SQUARES ESTIMATION TECHNIQUE .......................................................56

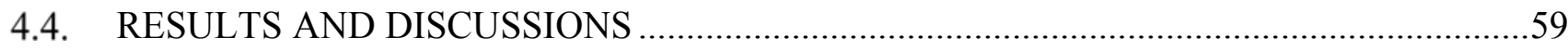

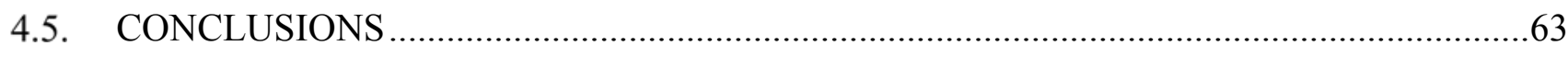

CHAPTER 5 - PERFORMANCE ANALYSIS OF SEVERAL GPS/GALILEO PRECISE POINT

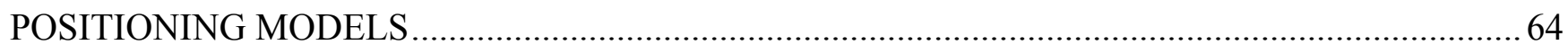

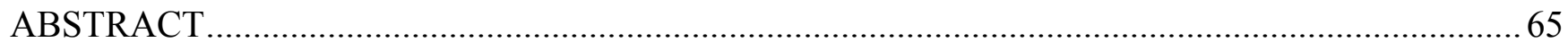

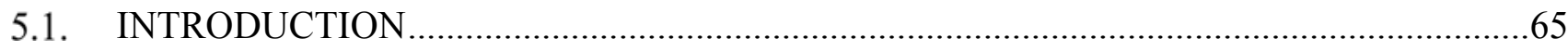


5.2. UN-DIFFERENCED GPS/GALILEO PPP MODELS .........................................................68

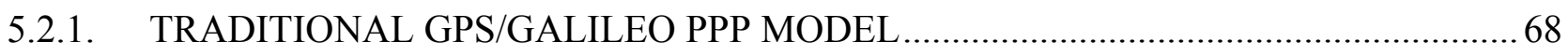

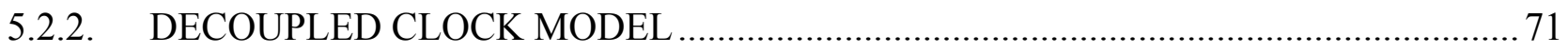

5.2.3. SEMI-DECOUPLED CLOCK GPS/GALILEO PPP MODEL .........................................76

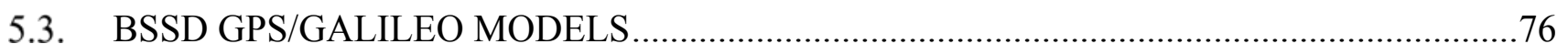

5.3.1. TRADITIONAL BSSD GPS/GALILEO PPP MODEL ……………………................. 76

5.3.2. BSSD DECOUPLED CLOCK GPS/GALILEO PPP MODEL ........................................78

5.3.3. BSSD SEMI-DECOUPLED CLOCK GPS/GALILEO PPP MODEL ............................... 80

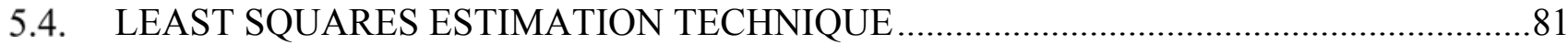

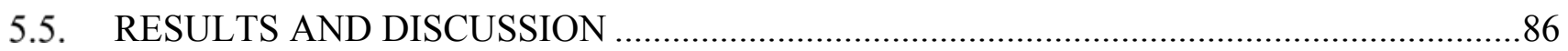

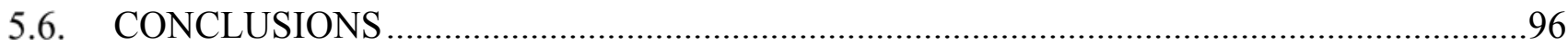

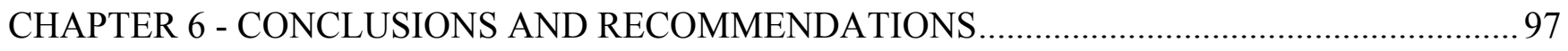

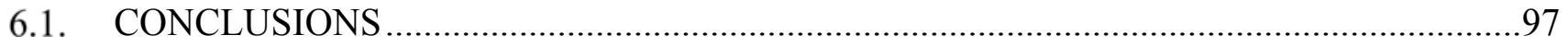

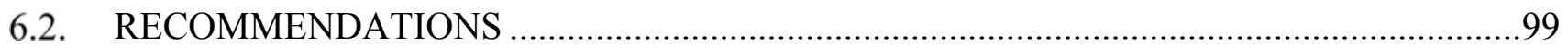

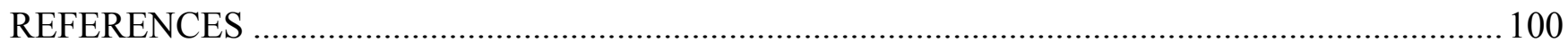




\section{LIST OF TABLES}

Table 2.1 Summary results of regression fitting functions with $95 \%$ confidence level ............. 21

Table 5. 1 Satellite clock correction difference between decoupled and IGS products ............. 75 


\section{LIST OF FIGURES}

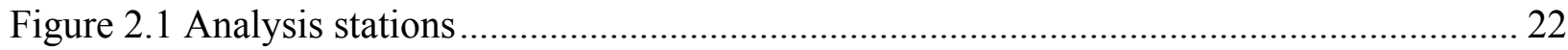

Figure 2.2 GPS PPP results using sine function stochastic model ...................................... 23

Figure 2.3 GPS PPP results using the newly developed stochastic model ............................... 23

Figure 2.4 GPS/Galileo PPP results using empirical stochastic model ................................. 24

Figure 2.5 GPS/Galileo PPP results using the newly developed stochastic model ................... 24

Figure 2.6 BSSD GPS PPP results using empirical sine function stochastic model .................. 25

Figure 2.7 BSSD GPS PPP results using the newly developed stochastic model ..................... 25

Figure 2.8 BSSD GPS/Galileo PPP results using empirical sine function stochastic model ....... 26

Figure 2.9 BSSD GPS/Galileo using the newly developed stochastic model .......................... 26

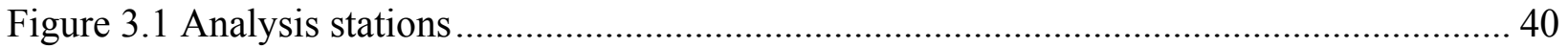

Figure 3.2 Un-differenced ionosphere-free GPS/Galileo PPP solution................................... 41

Figure 3.3 Un-differenced single frequency GPS/Galileo PPP solution ................................ 41

Figure 3.4 BSSD GPS/Galileo PPP solution using a GPS reference satellite .......................... 42

Figure 3.5 BSSD GPS/Galileo PPP solution using a Galileo reference satellite........................ 43

Figure 3.6 BSSD GPS/Galileo PPP solution using two reference satellites.............................. 44

Figure 3.7 Summary of standard deviations of obtained station coordinates .......................... 44

Figure 3.8 Summary of convergence times for all data sets analysed. (1) un-differenced model;

(2) BSSD model with a GPS satellite as a reference; (3) BSSD model with a Galileo satellite as a reference; (4) BSSD model with both a GPS and a Galileo as reference satellite...................... 45

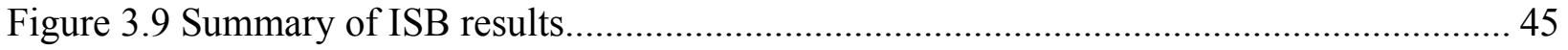


Figure 4.1 Analysis stations 59

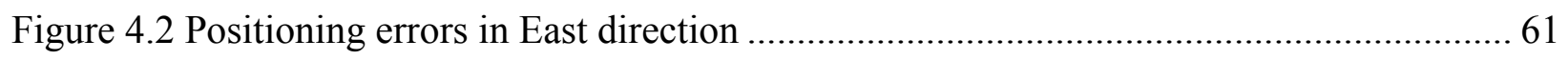

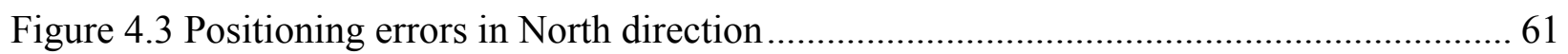

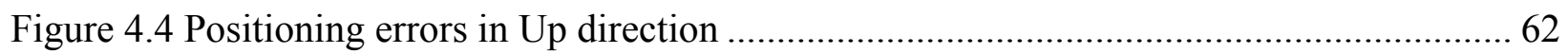

Figure 4.5 Summary of convergence times of all stations and analysis cases. (1) Un-differenced GPS model; (2) Un-differenced GPS/Galileo model; (3) BSSD model with a GPS satellite as a reference; (4) BSSD model with a Galileo satellite as a reference; (5) BSSD model with both a

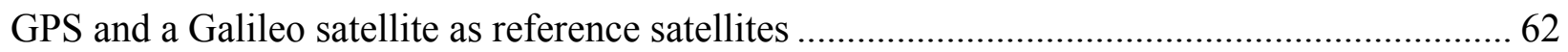

Figure 4.6 Positioning standard deviations in East, North, and Up directions ......................... 63

Figure 5. 1 Average 2014 IGS receiver DCB for GPS and Galileo signals ........................... 66

Figure 5. 2 Average IGS 2014 satellite DCB for both GPS/Galileo signals ........................... 66

Figure 5. 3 IGS and decoupled clock corrections 26 August 2012 ...................................... 74

Figure 5. 4 IGS and decoupled clock corrections 27 August 2012 ..................................... 75

Figure 5. 5 IGS and decoupled clock corrections 5 April 2013 ....................................... 75

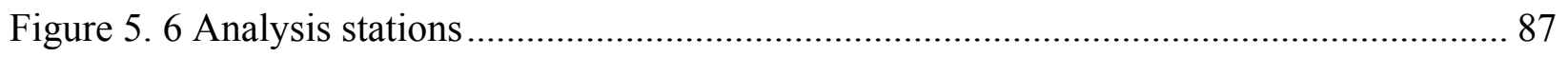

Figure 5. 7 Positioning results of the traditional GPS/Galileo PPP model ............................... 87

Figure 5. 8 Ambiguity parameters the traditional GPS/Galileo PPP model ............................ 87

Figure 5. 9 Positioning results of the GPS decoupled clock model..................................... 88

Figure 5. 10 Receiver clock errors of the GPS decoupled clock model ................................. 88

Figure 5. 11 Ambiguity parameters of the GPS decoupled clock model............................... 89

Figure 5. 12 Positioning results of the semi-decoupled clock PPP model............................... 89 
Figure 5. 13 Receiver clock errors of the semi-decoupled clock GPS/Galileo PPP model......... 90

Figure 5. 14 Ambiguity parameters of the semi-decoupled clock GPS/Galileo PPP model ....... 90

Figure 5. 15 Inter-system bias of the semi-decoupled clock GPS/Galileo PPP model............... 90

Figure 5. 16 Positioning results of the BSSD PPP tight combination model using (a) GPS reference satellite; and (b) Galileo reference satellite..................................................... 91

Figure 5. 17 Ambiguity parameters the BSSD PPP tight combination model using (a) GPS reference satellite; and (b) Galileo reference satellite.................................................. 91

Figure 5. 18 Positioning results of the BSSD PPP loose combination model .......................... 92

Figure 5. 19 Ambiguity parameters the BSSD PPP loose combination model ........................ 92

Figure 5. 20 Positioning results for BSSD semi-decoupled GPS/Galileo PPP model. (a) GPS reference satellite; and (b) Galileo reference satellite...................................................... 93

Figure 5. 21 Ambiguity parameters the semi-decoupled GPS/Galileo PPP model (a) GPS reference satellite; and (b) Galileo reference satellite................................................... 93 Figure 5. 22 Positioning results of the semi-decoupled per-constellation GPS/Galileo BSSD PPP

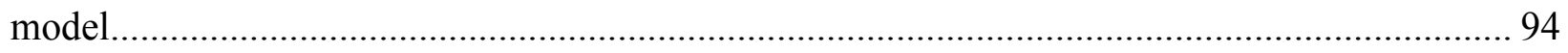
Figure 5. 23 Ambiguity parameters the semi-decoupled per-constellation GPS/Galileo BSSD PPP model 94

Figure 5. 24 Summary of convergence times of all stations and analysis cases (1) Un-differenced GPS model; (2) Un-differenced GPS/Galileo model; (3) Decoupled clock model using GPS observations only; (4) Semi-decoupled clock GPS/Galileo PPP model; (5) BSSD model with a GPS satellite as a reference; (6) BSSD model with a Galileo satellite as a reference; (7) BSSD model with both a GPS and a Galileo satellite as reference satellites; (8) BSSD semi-decoupled clock GPS/Galileo model with a GPS satellite as a reference; (9) BSSD semi-decoupled clock 
GPS/Galileo model with a Galileo satellite as a reference; (10) BSSD semi-decoupled clock GPS/Galileo model with both a GPS and a Galileo satellite as reference satellites

Figure 5. 25 Summary of positioning standard deviations in East, North, and Up directions of all stations and analysis cases. (1) Un-differenced GPS model; (2) Un-differenced GPS/Galileo model; (3) Decoupled clock model using GPS observations only; (4) semi-decouple clock GPS/Galileo PPP model; (5) BSSD model with a GPS satellite as a reference; (6) BSSD model with a Galileo satellite as a reference; (7) BSSD model with both a GPS and a Galileo satellite as reference satellites; (8) BSSD semi-decoupled clock GPS/Galileo model with a GPS satellite as a reference; (9) BSSD semi-decoupled clock GPS/Galileo model with a Galileo satellite as a reference; (10) BSSD semi-decoupled clock GPS/Galileo model with both a GPS and a Galileo satellite as reference satellites 96 


\section{ABBREVIATIONS}

BDT BeiDou time frame

BeiDou The Chinese navigation satellite system

BSSD Between-satellite single-difference

CODE Centre for Orbit Determination in Europe

DC Decoupled clock model

DCB Differential Code Biases

DoD Department of Defense

ESA European Space Agency

EU European Union

FDMA Frequency division multiple access

Galileo the European navigation satellite system

GGTO GPS to Galileo time offset

GIM Global Ionosphere Maps

GIOVE Galileo In-Orbit Validation Element

GLONASS Global Navigation Satellite System

GLONASST GLONASS Time system

GNSS Global Navigation Satellite System

GPS Global Positioning System

GPST GPS time frame

GST Galileo System Time

IF Ionosphere-Free

IGEX International GLONASS experiment

IGS International GNSS Service

ISB Inter-system bias

LC Linear Combination

MEGX Multi-GNSS Experiment

MEO Medium Earth Orbit 
NOAA National Oceanic and Atmospheric Administration

NRCan Natural Resources Canada

PPP Precise Point Positioning

SA Selective Availability

SoL Safety of life

TAI International Atomic Time

UHDs Un-calibrated hardware delays

US United States

USNO United States Naval Observatory

UTC Universal Coordinated Time 


\section{CHAPTER 1}

\section{INTRODUCTION}

This chapter provides necessary background information on the status of precise point positioning (PPP) techniques at the time when this research project was initiated. From this introduction emerge the motivations supporting the work conducted for this dissertation. The objectives of the research, the methodology utilized and the main contributions are also presented.

\subsection{BACKGROUND}

Presently, there exist four operational global navigation satellite systems (GNSS). These include the US global positioning system (GPS), the Russian global navigation satellite system (GLONASS), the European Galileo system, and the Chinese BeiDou system. Both GPS and GLONASS systems are fully operational however; Galileo and BeiDou systems are still under development. Combing the measurements of multiple systems can significantly improve the availability of a navigation solution, especially in urban areas.

GPS satellites transmit signals on three different frequencies, which are controlled by the GPS time frame (GPST). Currently, the GPS users can receive the modernized civil L2C and L5 signals. On the other hand, the GLONASS constellation has been fully recovered since October 2011. Presently, GLONASS operates at its full capability with 24 satellites in orbits, which enables a full global coverage. GLONASS transmits three signals on different main frequencies. However, unlike the other GNSS systems, each satellite transmits different frequencies based on

the frequency division multiple access (FDMA). GLONASS transmits its signals using the GLONASS Time system (GLONASST). Galileo satellite constellation foresees 27 operational and three spare satellites positioned in three nearly circular medium earth orbits (MEO). Galileo 
system transmits six signals on different frequencies using the Galileo time system (GST). Unlike GLONASS satellite system, Galileo and GPS have partial frequency overlaps, which simplify the dual-system integration. In addition, GPS and Galileo operators have agreed to measure and broadcast a GPS to Galileo time offset (GGTO) parameter, in order to facilitate the interchangeable mode (Melgard et al., 2013). The BeiDou navigation satellite system, being developed independently by China, is moving steadily forward towards completing the constellation. China has indicated a plan to complete the second generation of Beidou satellite system by expanding the regional service into global coverage. Beidou system transmits three signals on different frequencies using the BeiDou time frame (BDT). The BeiDou-2 system is proposed to consist of 30 medium Earth orbiting satellites and five geostationary satellites (Hofmann-Wellenhof et al., 2008; IAC, 2015; ESA, 2015; BeiDou, 2015).

Traditionally, differential GNSS techniques have been used in positioning applications requiring high accuracy. These techniques inherit their high accuracy from the fact that GNSS receivers in close proximity share the same errors and biases. The shorter the receiver separation is, the more similar are the errors and biases. As such, a major part of the GNSS error budget can be removed by differencing between the GNSS observables from these receivers. The major disadvantage of differential techniques, however, is the dependency on the measurements or corrections from a reference receiver or network. As such, differential techniques may not be a practical solution for some application scenarios due to the high cost or the lack of infrastructure (El-Rabbany, 2006). In addition, as the baseline length increases, the correlation between the errors at the receiver decreases and the errors would not cancel out sufficiently by differencing. As a result, the ambiguity parameters will not be fixed successfully and the positioning accuracy will deteriorate.

Over the past decade, a technique referred to as PPP, which employs a single GPS receiver, becomes more attractive to the users (Zumberge et al., 1997; Kouba and Héroux, 2001). Unlike classical GPS point positioning, which uses the pseudorange observations from four or more visible satellites and the broadcast ephemeris to obtain the user's instantaneous position, PPP attempts to account for all the GPS errors and biases (El-Rabbany, 2006). After deactivation of the selective availability (SA) in May 2000 and the production of precise satellite orbit and clock corrections, centimeter to decimeter positioning accuracy became possible with standalone GPS 
receivers. In addition to being cost effective, the PPP method provides accuracy level comparable to that of differential positioning.

Unfortunately, the use of a single constellation limits the number of visible satellites, especially in urban areas, which affects the PPP solution. Recently, a number of researchers showed that combining GPS and Galileo observations in PPP solution enhances the positioning convergence and precision in comparison with the GPS-only PPP solution (Afifi and El-Rabbany, 2013; Melgard et al., 2013). At present, the IGS-MEGX network provides the GNSS users with precise clock and orbit products to all currently available satellite systems (Montenbruck et al., 2014). This makes it possible to obtain a PPP solution by combining the observations of two or more GNSS constellations. This research focuses on combining the GPS and Galileo observations in a PPP model.

\subsubsection{GPS PRECISE POINT POSITIONING}

PPP was first introduced by researchers at the Jet Propulsion Laboratory (Zumberge et al., 1997). Since the introduction of the PPP technique, many studies have been performed on the accuracy and convergence time of the un-differenced GPS PPP model (see for example, Kouba and Héroux, 2001; Colombo et al., 2004; Ge at el., 2008; Collins at al., 2010). Generally, the accuracy of PPP depends on the ability to mitigate all errors and biases. These errors can be categorized as satellite-related errors, signal propagation-related errors and receiver/antennarelated errors (El-Rabbany, 2006). GNSS errors attributed to the satellites include satellite clock errors, orbital errors, satellite hardware delay, satellite antenna phase center variation, and satellite initial phase bias. Errors attributed to signal propagation include the delays of the GNSS signal as it passes through the ionospheric and tropospheric layers. Errors attributed to receiver/antenna configuration include, among others, the receiver clock errors, multipath error, receiver noise, receiver hardware delay, receiver initial phase bias, and receiver antenna phase center variations. Other biases have to be considered such as the effects of ocean loading (Bos and Scherneck, 2014; IERS, 2010), Earth tide (Kouba, 2009), carrier-phase windup (Leick, 2004; Wu et al., 1993), Sagnac (Kaplan and Hegarty, 2006), relativity (Hofmann-Wellenhof et al., 2008), and satellite and receiver antenna phase-center variations (Dow et al. 2009). 
Kouba and Heroux (2001) introduced a PPP model, which employs the traditional un-differenced dual-frequency pseudorange and carrier-phase measurements to form first-order ionosphere-free linear combinations. Their model, which obtains the ambiguity parameters as real-valued numbers, requires about 30 minutes for the solution to convergence to a submeter level accuracy. This relatively long convergence time is caused by the presence of un-calibrated hardware delays, which destroy the integer nature of the ambiguity parameters (Collins at al., 2010; Ge et al. 2008; Mercier and Laurichesse 2008; Kouba and Héroux, 2001). However, recent research has demonstrated that the correct integer values for the ambiguity parameter can be recovered if the satellites hardware delays can be calibrated (Collins at al., 2010; Ge et al. 2008; Laurichesse et al. 2009).

Between-satellite single difference (BSSD) GPS PPP models have also been developed a number of researchers (Elsobeiey and El-Rabbany, 2014; Colombo et al., 2004). BSSD GPS PPP model cancels out all receiver-related errors, including the receiver un-calibrated hardware delays, which significantly improves the convergence time as shown in Elsobeiey and El-Rabbany (2014) and Colombo et al. (2004). By cancelling out the receiver related error, both convergence time and precision of the PPP are improved as the case of the BSSD PPP model (Afifi and ElRabbany, 2015; Elsobeiey and El-Rabbany, 2014). In addition, using the BSSD model with the GPS decoupled clock corrections applied improves the precision of the estimated parameters by about 10\% compared with the GPS BSSD model (Elsobeiey and El-Rabbany, 2014).

\subsubsection{STOCHASTIC MODELING OF THE GNSS SIGNALS}

Generally, the mathematical model for GNSS PPP consists of two parts, namely functional and stochastic models. The functional part describes the geometrical relationship of the parameters of the PPP model, while the stochastic part describes the statistical (or stochastic) properties of the residual component in the functional model. The stochastic model is represented by the covariance matrix of the observations in the estimation model. The functional models related to PPP have been extensively studied by a number of researchers. This, however, is not the case with the more complex stochastic models, especially for the Galileo signals. Often, a simplified 
empirical stochastic model is used in GNSS positioning, which assumes that all the GNSS observables are statistically independent and of the same quality. This, in turn, leads to an overestimation of the estimated parameters (El-Rabbany, 1994). On the other hand, using the proper stochastic modelling of the GNSS signals improves the PPP solution precision and convergence time as shown in Afifi and El-Rabbany (2013). Most existing GNSS stochastic models use empirical functions such as the sine, cosine, exponential and polynomial functions. Typically, these stochastic models are functions of the satellite elevation angles (Elsobeiey and El-Rabbany, 2010). Unfortunately, existing empirical stochastic models may not be valid for all receiver types and GNSS signal frequencies. As such, it is essential that new stochastic models are developed for the new GNSS signal such as Galileo signals.

In order to determine the stochastic characteristics of the residual errors, two tests are usually carried out, namely the zero and short baselines tests. The zero baseline test uses one antenna connected by a signal splitter that feeds two or more GNSS receivers. Several receiver problems can be investigated by using the zero baseline test such as inter-channel biases and cycle slips. The use of a single antenna cancels out all systematic errors such as multipath and the preamplifier's noise. On the other hand, the short baseline test uses two receivers of a few meters apart and is usually carried out over two consecutive days when a single GNSS constellation is used. In this case, the double difference residuals of one day would contain the system noise and the multipath effect. As the multipath effect repeats almost every day for the GPS system, differencing the double difference residuals over two consecutive days cancels out the multipath effect and leaves a scaled system noise (El-Rabbany, 2006). However, multipath effect is not repeatable for the Galileo satellite system as the satellites take about 14 hours 4 minutes 41 seconds to orbit the Earth (Hofmann-Wellenhof et al., 2008).

In this research, a receiver system noise test is performed to determine the stochastic characteristics of Galileo E1 and E5a. As a by-product, the stochastic characteristics of the legacy GPS P1 code were obtained. To develop the stochastic model for Galileo signals, the pseudorange and carrier phase measurements of each receiver are first differenced, which cancels out the geometric term, satellite and receiver clock error, and tropospheric delay, This operation leaves the satellite and receiver hardware delays, ionosphere delay, the ambiguity 
parameter and the system noise. The noise parameters in the differenced data series are essentially those of the pseudorange observations. The phase measurement noise is usually neglected due to its small size compared to that of the pseudorange measurements (Elsobeiey and El-Rabbany, 2010). The receiver hardware delay is assumed to be stable over the observation period (i.e., usually within several hours), while the ambiguity parameter and initial phase bias are constants for a continuous session of measurements (Hofmann-Wellenhof et al., 2008). As such, they can be removed from the model through differencing with respect to the first value in the data series. The data series developed are divided into a certain number of bins depending on the satellite elevation angle. The standard deviation of the differenced system noise each bin is estimated. A least squares regression analysis is performed to obtain the best-fit model of the estimated standard deviations.

\subsubsection{GPS/GALILEO PRECISE POINT POSITIONING}

As indicated earlier, combining two satellite constellations offers more visible satellites to users, which in turn enhances the satellite geometry and is expected to improve the overall positioning solution (Hofmann-Wellenhof et al., 2008). However, combining the GPS and Galileo observations comes at the expense of introducing additional biases to the observation mathematical models such as GGTO due to the fact that each system uses a different time frame. GPS system uses the GPS time system, which is referenced to coordinated universal time (UTC) as maintained by the US Naval Observatory (USNO). On the other hand, Galileo satellite system the Galileo system time (GST), which is a continuous atomic time scale with a nominal constant offset with respect to the international atomic time (TAI) (Hofmann-Wellenhof et al., 2008). Recently, the European Space Agency (ESA) estimated the GGTO, which was found to be approximately $50 \mathrm{~ns}$, or equivalently $15 \mathrm{~m}$ range error (ESA, 2013). As well, the IGS estimated the inter-system bias (ISB) of the GPS/Galileo systems, which represents the difference between the ionosphere free linear combination of the receiver differential code biases (DCB) of both GPS and Galileo systems, at different stations with different receiver types. The published IGSMGEX daily results for the ISB indicate that value of the ISB is receiver type dependent, with a magnitude ranging from -30 to 60 nanoseconds. 
A combined GPS/Galileo PPP model can be formed using either of the un-differenced or BSSD modes. Afifi and El-Rabbany (2013) showed that combining GPS L1 and Galileo E1 observations in an un-differenced single-frequency PPP solution enhances the positioning solution convergence and precision in comparison with GPS-only PPP solution. Melgard et al. (2013) showed that combining multi-constellation in an un-differenced PPP solution improves the positioning accuracy, especially when the system biases are calibrated. In addition, Afifi and El-Rabbany (2014) showed that combining Galileo signals E1/E5a with the GPS L1/L2 signals in un-differenced dual-frequency ionosphere-free linear combinations improves the convergence time by $25 \%$ with a sub-decimeter accuracy level in comparison with the GPS-only PPP results. In addition, they showed that the inter-system bias is largely constant over a one-hour observation time span, which they used in their analysis, with a magnitude ranging from 30 to 60 nanoseconds depending on the GNSS receiver type.

Two scenarios are considered when forming the BSSD linear combination for the combined GPS/Galileo model, namely a tight and a loose combination. In the first scenario, either a GPS or a Galileo satellite is selected as a reference for both GPS and Galileo observables. The second scenario, which is commonly referred to as per-constellation combination, considers two reference satellites: a GPS reference satellite for the GPS observables and a Galileo satellite for the Galileo observables (Odijk and Teunissen, 2013). Afifi and El-Rabbany (2015) showed that combining GPS and Galileo observations in BSSD PPP model improves the precision of the estimated parameters by about $50 \%$ and $25 \%$, in comparison with the un-differenced GPS-only model, when the BSSD loose and the tight combinations are used, respectively. In addition, the solution convergence time is reduced to 10 minutes for both BSSD scenarios, which represents about 50\% improvement in comparison with the GPS-only PPP solution. 


\subsection{OBJECTIVES}

The main objective of this Ph.D. research is to develop a PPP model that combines GPS and Galileo observations in order to improve both the precision and the convergence time of the PPP solution. This will be fulfilled through a number of tasks, which can be summarized as follows:

1. To develop a stochastic model for the Galileo E1 and E5a signals.

2. To estimate the GPS and Galileo ISB for both single and dual frequency PPP model.

3. To develop a combined single frequency GPS and Galileo PPP algorithm for both undifferenced and between satellite single difference.

4. To develop a combined dual frequency GPS and Galileo PPP algorithm for both undifferenced and between satellite single difference.

5. To develop a combined GPS and Galileo PPP model, which considers the effect of the un-calibrated hardware delays effect on the ambiguity parameters. 


\subsection{RESEARCH CONTRIBUTIONS}

The main contributions made in this research can be summarized as follows:

1. Developed a stochastic model for the Galileo E1 and E5a signals, which improved the convergence time of GPS/Galileo L1/E1 PPP by up to $30 \%$.

2. Estimating the inter-system bias for both single and dual frequency GPS and Galileo PPP model.

3. Developed a combined un-differenced single frequency GPS and Galileo PPP model.

4. Developed a combined single frequency GPS and Galileo between satellite single difference PPP model. The results of the developed model show convergence time improvement of the single-frequency GPS/Galileo PPP solution by $35 \%$ and $15 \%$ when BSSD with tight and loose combinations are used, respectively.

5. Developed a combined dual frequency GPS/Galileo PPP model using un-differenced L1/L2 and E1/E5 a of GPS and Galileo signals, respectively. The results of the developed model show convergence time improvement by about $25 \%$ in comparison with the GPS-only PPP.

6. Developed a combined dual-frequency BSSD GPS/Galileo PPP model. The results of the developed model show that the precision of the estimated parameters improved by about $50 \%$ and $25 \%$, in comparison with the un-differenced GPS-only model, when the loose and the tight combinations are used, respectively.

7. Developed semi-decoupled clock GPS/Galileo PPP model that improves the convergence time by about $25 \%$ in comparison with the un-differenced GPS-only model. In addition, the semi-decoupled GPS/Galileo PPP model improves the solution precision by about $25 \%$ compared to the traditional un-differenced GPS/Galileo PPP model. 


\subsection{DISSERTATION OUTLINE}

This dissertation follows a manuscript style approach.

Chapter 2 defines the basis of the stochastic modeling for Galileo E1 and E5a signals. In this chapter, the stochastic characteristics of Galileo E1 and E5a and GPS L1 signals are determined using short baseline tests. The accuracy of the developed stochastic model is verified through single-frequency GPS/Galileo PPP tests in both un-differenced and BSSD modes. This chapter was published as:

Afifi, A., El-Rabbany, A. (2014). Single frequency GPS/Galileo precise point positioning using un-differenced and between-satellite single difference measurements. Geomatica, 68(3), 195-205. doi: 10.5623/cig2014-304

This paper is based on three conference proceedings:

Afifi, A., El-Rabbany, A. (2014). Precise point positioning using combined GPS and Galileo observations. The XXV International FIG Congress, June 16-21, 2014, Kuala Lumpur, Malaysia.

Afifi, A., El-Rabbany, A. (2013). Integrating GPS/Galileo systems in single frequency precise point positioning. Joint conference of the Canadian institute of Geomatics annual conference and the international conference on Earth observation for global changes. June 5-7, 2013 Toronto, Ontario

Afifi, A., El-Rabbany, A. (2013). A New Stochastic Model for Galileo E1 Signal. Joint conference of the Canadian institute of Geomatics annual conference and the international conference on Earth observation for global changes. June 5-7, 2013 Toronto, Ontario 
Chapter 3 develops a single-frequency PPP model using GPS and Galileo observations in BSSD mode. In this chapter, two scenarios are considered when forming the GPS and Galileo BSSD linear combination, namely a tight and a loose combination. In the first scenario, either a GPS or a Galileo satellite is selected as a reference for both GPS and Galileo observables. The second scenario considers two reference satellites: a GPS reference satellite for the GPS observables and a Galileo satellite for the Galileo observables. This chapter was published as:

Afifi, A., El-Rabbany, A. (2015). An improved model for single frequency GPS/Galileo precise point positioning. Positioning Journal, 6(2), 7-21, doi: 10.4236/pos.2015.62002.

This paper is based on one conference proceedings:

Afifi, A., El-Rabbany, A. (2013). A combined precise point positioning solution using GPS and Galileo measurements. International symposium on global navigation satellite systems ISGNSS. October 22-25, 2013 Istanbul, Turkey

Chapter 4 develops a dual-frequency GPS/Galileo PPP model, which uses un-differenced and BSSD (with both loose and tight combinations) models. It is originally published as:

Afifi, A., El-Rabbany, A. (2015). An improved between-satellite single-difference precise point positioning model for combined GPS/Galileo observations. Journal of Applied Geodesy, 9(2), 101-111, doi: 10.1515/jag-2014-0030.

This paper is based on one conference proceedings:

Afifi, A., El-Rabbany, A. (2014). Improved model for precise point positioning with dual frequency GPS/Galileo observables. The International Archives of the Photogrammetry, Remote Sensing and Spatial Information Sciences, Volume XL-2, 2014 ISPRS Technical Commission II Symposium, 6 - 8 October 2014, Toronto, Canada 
Chapter 5 examines the performance of several precise point positioning (PPP) models, which combine dual-frequency GPS/Galileo observations in the un-differenced and between-satellite single-difference (BSSD) modes. These include the traditional un-differenced model, the decoupled clock model, the semi-decoupled clock model, and between-satellite single-difference model. The results were published as:

Afifi, A., El-Rabbany, A. (2015). Performance analysis of several GPS/Galileo precise point positioning models, Sensors, 15(6), 14701-14726; doi:10.3390/s150614701

Chapter 6 presents the conclusions of the dissertation findings and suggests further paths to explore.

Note that changes to the notation of each paper were made to assure uniformity within this dissertation. 


\section{CHAPTER 2}

\section{SINGLE FREQUENCY GPS/GALILEO PRECISE POINT POSITIONING USING UN- DIFFERENCED AND BETWEEN SATELLITE SINGLE DIFFERENCE MEASUREMENTS}

This chapter defines the basis of the stochastic modeling for Galileo E1 and E5a signals. In this chapter, the stochastic characteristics of Galileo E1 and E5a and GPS L1 signals are determined using short baseline tests. The accuracy of the developed stochastic model is verified through single-frequency GPS/Galileo PPP tests in both un-differenced and BSSD modes. This chapter was published as:

Afifi, A., El-Rabbany, A. (2014). Single frequency GPS/Galileo precise point positioning using un-differenced and between-satellite single difference measurements. Geomatica, 68(3), 195-205. doi: 10.5623/cig2014-304

Modifications to the original manuscript were made only for proper identification of sections, figures and tables, as well as to assure the uniformity of symbol and equation notation throughout this dissertation. 


\begin{abstract}
We develop a new precise point positioning (PPP) model for combined GPS/Galileo singlefrequency observations. Both un-differenced and between-satellite single-difference (BSSD) modes are considered. Although it improves the solution availability and accuracy, combining GPS and Galileo observables introduces additional biases that must be modelled. These include the GPS-to-Galileo time offset and the inter-system bias. Additionally, to take full advantage of the Galileo E1 signal, it is essential that its stochastic characteristics are rigorously modelled. In this paper, various sets of GPS and Galileo measurements collected at two stations with short separation were used to investigate the stochastic characteristics of Galileo E1 signal. As a byproduct, the stochastic characteristics of the legacy GPS P1 code was obtained and then used to verify the developed stochastic model of the Galileo signal. It is shown that sub-decimeter level accuracy is possible through our single-frequency GPS/Galileo PPP model. As well, the addition of Galileo improves the PPP solution convergence by about 30\% in comparison with GPS-only solution.
\end{abstract}

\title{
2.1. INTRODUCTION
}

Traditionally, ionosphere-free linear combinations of GPS carrier-phase and pseudorange measurements were used for precise point positioning (PPP). Both un-differenced and betweensatellite single difference measurements have been used (see for example, Kouba and Héroux, 2001; Colombo et al., 2004; Ge at el., 2008; Collins at al., 2010, Zumberge et al, 1997). PPP has been proven to be capable of providing positioning solution at the sub-decimeter level in static mode. More recently, Elsobeiey and El-Rabbany (2013) showed that about 50\% improvement in the PPP solution convergence time can be achieved with GPS dual frequency ionosphere-free BSSD.

A drawback of a single satellite constellation such as GPS is the availability of sufficient number of visible satellites in urban areas. Galileo satellite system offers additional visible satellites to the user, which is expected to enhance the satellite geometry and the overall PPP solution when combined with GPS (Hofmann-Wellenhof et al., 2008). As shown in Afifi and El-Rabbany (2013), combining GPS and Galileo observations in a PPP solution enhances the positioning 
solution convergence and precision in comparison with GPS-only PPP solution. This, however, requires rigorous modelling of all errors and biases.

Generally, the mathematical model for GNSS PPP consists of two parts, namely functional and stochastic models. The functional part describes the physical or geometrical characteristics of the parameters of the PPP model, while the stochastic part describes the statistical (or stochastic) properties of the un-modelled residual components in the functional part. Often, a simplified empirical stochastic model is used in GNSS positioning, which assumes that all GNSS observables are statistically independent and of the same quality. This, in turn, leads to an overestimation of the estimated parameters (El-Rabbany, 1994). As shown in Afifi and ElRabbany (2013), using the proper stochastic modelling of the GNSS signals leads to improving the PPP solution precision and convergence time.

This paper develops a PPP model, which combines GPS and Galileo single-frequency observables using both un-differenced and BSSD modes. All errors and biases are rigorously accounted for. Un-modelled residual components are accounted for using stochastic models. A new stochastic model for the Galileo signal is also developed, which does not exist at present. It is shown that sub-decimeter level accuracy is possible through our single-frequency GPS/Galileo PPP model. As well, the addition of Galileo improves the PPP solution convergence by about $30 \%$ in comparison with GPS-only solution.

\subsection{UN-DIFFERENCED GPS/GALILEO MODEL}

GNSS observations are affected by errors and biases, which can be categorized as satelliterelated errors, signal propagation-related errors and receiver/antenna-related errors (El-Rabbany, 2006; Hofmann-Wellenhof et al., 2008; Leick, 1995). GNSS errors attributed to the satellites include satellite clock errors, orbital errors, satellite hardware delay, satellite antenna phase centre variation, and satellite initial phase bias. Errors attributed to signal propagation include the delays of the GNSS signal as it passes through the ionospheric and tropospheric layers. Errors attributed to receiver/antenna configuration include, among others, the receiver clock errors, multipath error, receiver noise, receiver hardware delay, receiver initial phase bias, and receiver antenna phase center variations. 
In addition to the above errors and biases, combining GPS and Galileo observation in a PPP model introduces additional errors such as GPS to Galileo time offset (GGTO) due to the fact that each system uses a different time frame. GPS system uses the GPS time system, which is referenced to coordinated universal time (UTC) as maintained by the US Naval Observatory (USNO). On the other hand, Galileo satellite system the Galileo system time (GST), which is a continuous atomic time scale with a nominal constant offset with respect to the international atomic time (TAI) (Hofmann-Wellenhof et al., 2008). As well, GPS and Galileo use different reference frames, which should be considered in the combined PPP solution. The mathematical models of GPS and Galileo observables, code and carrier phase, can be written respectively as:

$$
\begin{gathered}
\mathrm{P}_{\mathrm{G}}=\rho_{G}\left(t_{G},(t-\tau)_{G}\right)+\mathrm{c}\left[\mathrm{dt}_{\mathrm{r}}\left(t_{G}\right)-\mathrm{dt}^{\mathrm{s}}(t-\tau)_{G}\right]+T_{G}+I_{G}+c\left[d_{r}\left(t_{G}\right)+d^{s}(t-\tau)_{G}\right]+d_{m p}+e_{P G} \\
\mathrm{P}_{\mathrm{E}}=\rho_{E}\left(t_{E},(t-\tau)_{E}\right)+\mathrm{c}\left[\mathrm{dt}_{\mathrm{r}}\left(t_{E}\right)-\mathrm{dt}^{\mathrm{s}}(t-\tau)_{E}\right]+T_{E}+I_{E}+c\left[d_{r}\left(t_{E}\right)+d^{s}(t-\tau)_{E}\right]+d_{m p}+e_{P E} \\
\Phi_{\mathrm{G}}=\rho_{G}\left(t_{G},(t-\tau)_{G}\right)+\mathrm{c}\left[\mathrm{dt}_{\mathrm{r}}\left(t_{G}\right)-\mathrm{dt}^{\mathrm{s}}(t-\tau)_{G}\right]+T_{G}-I_{G}+ \\
c\left[\delta_{r}\left(t_{G}\right)+\delta^{s}(t-\tau)_{G}\right]+\lambda\left[N_{G}+\phi_{r}\left(t_{0}\right)-\phi^{s}\left(t_{0}\right)\right]+\delta_{m p}+\varepsilon_{\varphi G} \\
\Phi_{\mathrm{E}}=\rho_{E}\left(t_{E},(t-\tau)_{E}\right)+\mathrm{c}\left[\mathrm{dt}_{\mathrm{r}}\left(t_{E}\right)-\mathrm{dt}^{\mathrm{s}}(t-\tau)_{E}\right]+T_{E}-I_{E}+ \\
c\left[\delta_{r}\left(t_{E}\right)+\delta^{s}(t-\tau)_{E}\right]+\lambda\left[N_{E}+\phi_{r}\left(t_{0}\right)-\phi^{s}\left(t_{0}\right)\right]+\delta_{m p}+\varepsilon_{\varphi E}
\end{gathered}
$$

where the subscript $G$ refers to the GPS satellite system and the subscript $E$ refers to the Galileo satellite system; $\mathrm{P}_{\mathrm{G}}$ and $\mathrm{P}_{\mathrm{E}}$ are pseudoranges for the GPS and Galileo systems, respectively; $\Phi_{\mathrm{G}}$ and $\Phi_{\mathrm{E}}$ are the carrier phase measurements of the GPS and Galileo systems, respectively; $\operatorname{dt}_{\mathrm{r}}(\mathrm{t})$, $\mathrm{dt}^{\mathrm{s}}(\mathrm{t}-\tau)$ are the clock error for receiver at reception time $\mathrm{t}$ and satellite at transmitting time $\mathrm{t}-\tau$, respectively; $d_{r}(t), d^{s}(t-\tau)$ are frequency dependent code hardware delay for receiver at reception time $\mathrm{t}$ and satellite at transmitting time $\mathrm{t}-\tau$, respectively; $\delta_{\mathrm{r}}(\mathrm{t}), \delta^{\mathrm{s}}(\mathrm{t}-\tau)$ are frequency-dependent carrier phase hardware delay for receiver at reception time $\mathrm{t}$ and satellite at transmitting time $\mathrm{t}-\tau$, respectively; $T$ is the tropospheric delay; $I$ is ionospheric delay; $d_{m p}$ is code multipath effect; $\delta_{m p}$ is the carrier phase multipath effect; $\lambda$ is the wavelengths of carrier frequencies, respectively; $\Phi_{\mathrm{r}}\left(\mathrm{t}_{0}\right), \Phi^{s}\left(\mathrm{t}_{0}\right)$ are frequency-dependent initial fractional phases in the receiver and satellite channels; $N$ is the integer number of cycles for the carrier phase measurements, respectively; $\mathrm{c}$ is the speed of light in vacuum; and $\rho$ is the true geometric range from receiver at reception time to satellite at transmission time; ep, $\varepsilon_{\Phi}$ are the relevant noise and un-modelled errors. 
Several organizations such as the International GNSS Service (IGS) and the Cooperative Network for GIOVE Observations (CONGO) network provide the user with precise products, including precise satellite orbit and clock corrections. IGS precise satellite orbit and clock corrections contain the satellite hardware delay of the ionosphere-free linear combination of GPS L1 and L2 signals (Kouba, 2009). On the other hand, CONGO satellite precise orbital and clock corrections include the satellite hardware delay of the ionosphere-free linear combination of Galileo E1 and E5a signals (Montenbruck at al., 2009). In this research, the precise orbit and satellite clock corrections from the CONGO network are used for both GPS and Galileo satellites. In addition, the GPS receiver hardware delay is lumped to the receiver clock error. This, in turn introduces a new term in the Galileo observation equations, which represents the difference between the satellite hardware delays of GPS and Galileo signals. A new unknown (ISB) is considered in our model to account for the system time offset as well as the new satellite hardware difference term as shown in equations 2.7 and 2.8. The receiver and satellite hardware delays can be lumped to the receiver clock error and to the GGTO as all of these errors are timing errors. Equations 2.5 to 2.8 show the final combined GPS and Galileo PPP model.

$$
\begin{aligned}
& \mathrm{P}_{\mathrm{G}}=\rho_{G}+\mathrm{c}\left[\mathrm{dt}_{\mathrm{r}}-\mathrm{dt}_{\mathrm{IGS}}^{\mathrm{s}}\right]+T_{G}+I_{G}+e_{P G} \\
& \Phi_{\mathrm{G}}=\rho_{G}+\mathrm{c}\left[\mathrm{dt}_{\mathrm{r}}-\mathrm{dt}_{\mathrm{IGS}}^{\mathrm{s}}\right]+T_{G}-I_{G}+\lambda \widetilde{N}_{G}+\varepsilon_{\varphi G} \\
& \mathrm{P}_{\mathrm{E}}=\rho_{E}+\mathrm{c}\left[\mathrm{dt}_{\mathrm{r}}-\mathrm{dt}_{\mathrm{CON}}^{\mathrm{s}}\right]+I S B+T_{E}+I_{E}+e_{P E} \\
& \Phi_{\mathrm{E}}=\rho_{E}+\mathrm{c}\left[\mathrm{dt}_{\mathrm{r}}-\mathrm{dt}_{\mathrm{CON}}^{\mathrm{s}}\right]+I S B+T_{E}-I_{E}+\lambda \widetilde{N}_{E}+\varepsilon_{\varphi E}
\end{aligned}
$$

where $\widetilde{N}$ is the ambiguity parameter including frequency-dependent initial fractional phases in the receiver and satellite channels; ISB is the newly introduced unknown parameter.

\subsection{BSSD GPS/GALILEO MODE}

Differencing the observations between satellites cancels out most receiver-related errors, including receiver clock error, receiver hardware delays, and non-zero initial phase bias (ElRabbany, 2006; Hofmann-Wellenhof et al., 2008; Leick, 1995). In this research a GPS satellite is used to be a reference satellite for both GPS and Galileo satellites. As a result, all differenced observations will be mathematically correlated. A simple way of accounting for the 
mathematical correlation could be done through the covariance matrix which, in our case will be a fully populated matrix. The weight matrix, which is needed in the least-squares estimation, can be obtained by scaling the inverse of the covariance matrix. Assuming a unit scale factor and $n_{s}$ visible satellites, the weight matrix for one epoch $P(t)$ can be written as:

$$
P(t)=\sum_{B S S D}^{-1}=\frac{1}{n_{S} \sigma^{2}}\left[\begin{array}{ccccc}
\left(n_{s}-1\right) & -1 & -1 & -1 & -1 \\
-1 & \left(n_{s}-1\right) & -1 & -1 & -1 \\
-1 & -1 & \left(n_{s}-1\right) & -1 & -1 \\
-1 & -1 & -1 & \left(n_{s}-1\right) & -1 \\
-1 & -1 & -1 & -1 & \left(n_{s}-1\right)
\end{array}\right]_{\left(n_{s}-1\right) x\left(n_{s}-1\right)}
$$

As can be seen in Equation 2.9, the relative weight matrix of the observations in the proposed BSSD mathematical model is no longer diagonal matrix. When a GPS satellite is used as a reference in the BSSD mode, the design matrix A and the vector of unknown parameters $\mathrm{x}$ take the following forms:

$$
A=\left[\begin{array}{ccccccccc}
\frac{x_{0}-X^{1}}{\rho_{0}^{1}}-\frac{x_{0}-X^{2}}{\rho_{0}^{2}} & \frac{y_{0}-Y^{1}}{\rho_{0}^{1}}-\frac{y_{0}-Y^{2}}{\rho_{0}^{2}} & \frac{z_{0}-Z^{1}}{\rho_{0}^{1}}-\frac{z_{0}-Z^{2}}{\rho_{0}^{2}} & 0 & 0 & 0 & \cdots & 0 \\
\frac{x_{0}-X^{1}}{\rho_{0}^{1}}-\frac{x_{0}-X^{2}}{\rho_{0}^{2}} & \frac{y_{0}-Y^{1}}{\rho_{0}^{1}}-\frac{y_{0}-Y^{2}}{\rho_{0}^{2}} & \frac{z_{0}-Z^{1}}{\rho_{0}^{1}}-\frac{z_{0}-Z^{2}}{\rho_{0}^{2}} & 0 & N^{12} & 0 & \cdots & 0 \\
\frac{x_{0}-X^{1}}{\rho_{0}^{1}}-\frac{x_{0}-X^{3}}{\rho_{0}^{3}} & \frac{y_{0}-Y^{1}}{\rho_{0}^{1}}-\frac{y_{0}-Y^{3}}{\rho_{0}^{3}} & \frac{z_{0}-Z^{1}}{\rho_{0}^{1}}-\frac{z_{0}-Z^{3}}{\rho_{0}^{3}} & 0 & 0 & 0 & \cdots & 0 \\
\frac{x_{0}-X^{1}}{\rho_{0}^{1}}-\frac{x_{0}-X^{3}}{\rho_{0}^{3}} & \frac{y_{0}-Y^{1}}{\rho_{0}^{1}}-\frac{y_{0}-Y^{3}}{\rho_{0}^{3}} & \frac{z_{0}-Z^{1}}{\rho_{0}^{1}}-\frac{z_{0}-Z^{3}}{\rho_{0}^{3}} & 0 & 0 & N^{13} & \cdots & 0 \\
\vdots & \vdots & \vdots & \vdots & \vdots & \cdots & \vdots \\
\frac{x_{0}-X^{1}}{\rho_{0}^{1}}-\frac{x_{0}-X^{n}}{\rho_{0}^{n}} & \frac{y_{0}-Y^{1}}{\rho_{0}^{1}}-\frac{y_{0}-Y^{n}}{\rho_{0}^{n}} & \frac{z_{0}-Z^{1}}{\rho_{0}^{1}}-\frac{z_{0}-Z^{n}}{\rho_{0}^{n}} & 1 & 0 & 0 & \cdots & 0 \\
\frac{x_{0}-X^{1}}{\rho_{0}^{1}}-\frac{x_{0}-X^{n}}{\rho_{0}^{n}} & \frac{y_{0}-Y^{1}}{\rho_{0}^{1}}-\frac{y_{0}-Y^{n}}{\rho_{0}^{n}} & \frac{z_{0}-Z^{1}}{\rho_{0}^{1}}-\frac{z_{0}-Z^{n}}{\rho_{0}^{n}} & 1 & 0 & 0 & \cdots & N^{1 n}
\end{array}\right]_{2(\mathrm{n}-1) \times(\mathrm{n}+3)} \quad x=\left[\begin{array}{c}
\Delta x \\
\Delta y \\
\Delta Z \\
I S B \\
N^{12} \\
N^{13} \\
\vdots \\
\vdots \\
N^{1 n}
\end{array}\right]_{n+3}
$$

The additional system bias term appears in the Galileo observations equations only. Obviously, the related receiver errors are canceled out from the unknown vector. Consequently, the unknowns are the three coordinates of the receiver, $\Delta \mathrm{x}, \Delta \mathrm{y}$ and $\Delta \mathrm{z}$, the additional system bias term, and differenced ambiguities parameters $N^{\text {in }}$.

\subsection{SEQUENTIAL LEAST SQUARES ESTIMATION}

Sequential least-squares estimation technique is used in this research to get the best estimates in the least-squares sense. Equations 2.5 to 2.8 can be re-arranged for pseudorange and carrier phase observations after applying the ionospheric and tropospheric corrections as follows:

$f_{\mathrm{P}_{\mathrm{G}}}=\rho+\mathrm{cdt}_{\mathrm{r}}+e_{P G}-P_{G}=0$ 
$f_{\mathrm{P}_{\mathrm{E}}}=\rho+\mathrm{cdt} \mathrm{dt}_{\mathrm{r}}+I S B+e_{P E}-P_{E}=0$

$f_{\Phi_{\mathrm{G}}}=\rho+\mathrm{cdt} \mathrm{dt}_{\mathrm{r}}+\lambda \widetilde{N}_{G}+e_{P G}-\Phi_{G}=0$

$f_{\Phi_{\mathrm{E}}}=\rho+\mathrm{cdt} \mathrm{dt}_{\mathrm{r}}+\lambda \widetilde{N}_{E}+I S B+e_{P E}-\Phi_{E}=0$

The linearized form of Equations 2.11 to 2.14 around the initial parameter $\mathrm{x}^{0}$ and observables $l$ in matrix form can be written as:

$f(x, l)=A \Delta x-w-r=0$

The sequential least square estimation technique can then be written as:

$$
\begin{aligned}
& \Delta x_{i}=\Delta x_{i-1}+N_{i-1}^{-1} A_{i}^{* T}\left[P_{i, i}^{-1}+A_{i}^{*} N_{i-1}^{-1} A_{i}^{* T}\right]^{-1}\left[w_{i}^{*}-A_{i}^{*} \Delta x_{i-1}\right] \\
& N_{i}^{-1}=N_{i-1}^{-1}-N_{i-1}^{-1} A_{i}^{* T}\left[P_{i, i}^{-1}+A_{i}^{*} N_{i-1}^{-1} A_{i}^{* T}\right]^{-1} A_{i}^{*} N_{i-1}^{-1} \\
& A_{i}^{*}=A_{i}-C_{i, i-1} C_{i, i-1}^{-1} A_{i-1} \\
& w_{i}^{*}=w_{i}-C_{i, i-1} C_{i, i-1}^{-1} w_{i-1}
\end{aligned}
$$

where $A$ is the design matrix, which includes the partial derivatives of the observation equations with respect to the unknown parameters $X ; \Delta \mathrm{x}$ is the vector of corrections to the unknown parameters $\left(\Delta x=x-x^{0}\right) ; w$ is the misclosure vector; $r$ is the residuals victor; $C$ is the observations covariance matrix; $P$ is the observations weight matrix; $N$ is the matrix of the normal equations; $i$ is the epoch index.

To combine the GPS and Galileo observations in a PPP solution, it is essential that the statistical characteristics of the noise terms in the above equations are described using the proper stochastic model.

\subsection{STOCHASTIC MODEL DEVELOPMENT}

The receiver measurement noise results from the limitations of the receiver's electronics and can be determined through receiver calibration or test. Two tests are usually carried out to determine the system noise level, namely the zero and short baselines tests. The zero baseline test employs one antenna followed by a signal splitter that feeds two or more GPS receivers. Using the zero 
baseline test, several receiver problems can be investigated, such as inter-channel biases and cycle slips. The single antenna cancels out the real world systematic problems such as multipath in addition to the preamplifier's noise. The short baseline test, on the other hand, uses two receivers a few meters apart and is usually carried out over two consecutive days.

In this case, the double difference residuals of one day would contain the system noise and the multipath effect. As the multipath effect repeats every sidereal day for GPS system, differencing the double difference residuals of the two consecutive days cancels out the multipath effect and leaves the scaled system noise (El-Rabbany, 2006). However, multipath effect is not repeatable every sidereal day for the Galileo satellite system as the satellites take about 14 hours 4 minutes 41 seconds to orbit the Earth (Hofmann-Wellenhof et al., 2008).In this research, a short baseline test is used to determine the stochastic characteristics of the E1 signal, assuming that multipath does not exist. Usually, this test is performed using the same type of receivers. Unfortunately, in this research, two different receivers were available (Septentrio POLARX4TR and Trimple NETR9) for the test, which can observe the Galileo measurements. This, however, were considered when processing the data. The pseudorange and carrier phase equations can be rewritten as, assuming no multipath and dropping the time argument:

$$
\begin{aligned}
& \mathrm{P}_{\mathrm{i}}=\rho+\mathrm{c}\left[\mathrm{dt}_{\mathrm{r}}-\mathrm{dt}^{\mathrm{s}}\right]_{\mathrm{i}}+c\left[d_{r}+d^{s}\right]_{i}+T_{i}+I_{i}+e_{P i} \\
& \Phi_{\mathrm{i}}=\rho+\mathrm{c}\left[\mathrm{dt}_{\mathrm{r}}-\mathrm{dt}^{\mathrm{s}}\right]_{\mathrm{i}}+c\left[\delta_{r}+\delta^{s}\right]_{i}+T_{i}-I_{i}+\lambda \widetilde{N}+e_{\Phi i}
\end{aligned}
$$

Differencing the pseudorange and carrier phase equations of each receiver cancels out the geometric term, satellite and receiver clock error, and tropospheric delays, as shown in Equations (2.22) and (2.23). The remaining terms include the satellite and receiver hardware delays, ionosphere delay, the ambiguity parameter and the system noise.

$$
\begin{aligned}
& \Delta R_{1}=P_{\mathrm{R} 1}-\Phi_{\mathrm{R} 1}=\mathrm{c}\left[\mathrm{d}_{\mathrm{r}}-\mathrm{d}^{\mathrm{s}}\right]_{1}+c\left[\delta_{r}-\delta^{s}\right]_{1}+\Delta \lambda \tilde{N}_{1}+2 I+e_{P} \\
& \Delta R_{2}=P_{R 2}-\Phi_{R 2}=\mathrm{c}\left[\mathrm{d}_{\mathrm{r}}-\mathrm{d}^{\mathrm{s}}\right]_{2}+c\left[\delta_{r}-\delta^{s}\right]_{2}+\Delta \lambda \tilde{N}_{2}+2 I+e_{p}
\end{aligned}
$$

It should be pointed out that the noise parameters in Equations 2.22 and 2.23 are essentially those of the pseudorange measurements. The phase measurement noise has been neglected due to its 
small size compared to that of the pseudorange measurements (Elsobeiey and El-Rabbany, 2010). The receiver hardware delay is assumed to be stable over the observation period (four hours in this research). Data series representing the values of $\Delta R_{1}$ and $\Delta R_{2}$ over the entire observation session are generated. As the ambiguity parameter and initial phase bias remain constant as long as no signal loss occurred, they can be removed from the model through differencing with respect to the first value of the series. Using this approach, only the differenced system noise remains in the model.

In PPP, most of existing observation stochastic models are empirical functions such as sine, cosine, exponential and polynomial functions. Most of these stochastic models are functions of the satellite elevation angles (Leandro and Santos 2007). Unfortunately, existing stochastic models may not be valid for all receiver types and GNSS signal frequencies. As such, it is essential that new stochastic models are developed for the Galileo signal. The data series developed are divided into nine bins depending on the satellite elevation angle, starting from $0^{\circ}$ to $90^{\circ}$ with increments of $10^{\circ}$. The standard deviation of the differenced system noise for each bin is estimated (Elsobeiey and El-Rabbany, 2010). A least squares regression analysis is performed to obtain the best-fit model of the estimated standard deviations. Three empirical functions were tested for this purpose, namely an exponential, a polynomial and a rational model as shown in Table 2.1. The best-fit model is selected based on the goodness of fit test, i.e., the one with the largest $\mathrm{R}^{2}$ (R-squared) statistic (Draper, 2002).

Table 2.1 Summary results of regression fitting functions with $95 \%$ confidence level

\begin{tabular}{|c|c|c|c|c|c|c|c|c|c|}
\hline & \multicolumn{2}{|c|}{ Exponential function } & \multicolumn{3}{c|}{ Polynomial function } & \multicolumn{3}{c|}{ Rational function } \\
\cline { 2 - 11 } & \multicolumn{3}{|c|}{$S T D=\mathrm{a} \times e^{(-\mathrm{b} \times E L E)}+\mathrm{c}$} & \multicolumn{3}{c|}{$S T D=-\mathrm{a} \times E L E^{3}+\mathrm{b} \times E L E^{2}-\mathrm{c}$} & \multicolumn{2}{c|}{$S T D=\frac{\left(a \times E L E^{2}-b \times E L E+c\right)}{(E L E+d)}$} \\
\hline Signals & $\mathrm{E} 1$ & $\mathrm{E} 5 \mathrm{a}$ & $\mathrm{L} 1$ & $\mathrm{E} 1$ & $\mathrm{E} 5 \mathrm{a}$ & $\mathrm{L} 1$ & $\mathrm{E} 1$ & $\mathrm{E} 5 \mathrm{a}$ & $\mathrm{L} 1$ \\
\hline $\mathrm{a}$ & 0.6383 & 0.3692 & 0.6830 & $1.835 \mathrm{e}-6$ & $5.892 \mathrm{e}-6$ & $1.473 \mathrm{e}-6$ & $3.5 \mathrm{e}-3$ & $4.315 \mathrm{e}-3$ & $6.087 \mathrm{e}-3$ \\
\hline $\mathrm{b}$ & 0.0763 & 0.0753 & 0.0730 & $3.688 \mathrm{e}-4$ & $1.445 \mathrm{e}-4$ & $3.195 \mathrm{e}-4$ & 0.2703 & 0.5155 & 0.6533 \\
\hline $\mathrm{c}$ & 0.2150 & 0.0974 & 0.1751 & 0.02443 & 0.01556 & 0.0228 & 22.93 & 28.36 & 36.57 \\
\hline $\mathrm{d}$ & - & - & - & 0.7557 & 0.4014 & 0.7156 & 28.35 & 69.29 & 49.69 \\
\hline $\mathrm{R}^{2}$ & 0.9995 & 0.9993 & 0.9994 & 0.9988 & 0.9977 & 0.9990 & 0.9990 & 0.9977 & 0.9984 \\
\hline
\end{tabular}


where $E L E$ is the satellite elevation angle in degrees; STD is the observation standard deviation

Table 2.1 summarizes the results of all three tested functions. As shown, the exponential function was found to be the best-fitting model in the least-squares sense, which was selected in this research.

\subsection{RESULTS AND DISCUSSION}

To test our PPP model and to verify the determined stochastic models of the Galileo E1 signal, Natural Resources Canada (NRCan) GPSPace PPP software was modified to handle the Galileo observations in addition to the newly developed stochastic models. The GPS/Galileo PPP solution is also obtained using an existing empirical function, namely the sine function, which is compared with the PPP solution obtained with newly developed stochastic model. Four stations were used to verify our PPP model, two stations in North America (UNB and USN) and two in Europe (Dlft and GOPE) as shown in Figure 2.1.

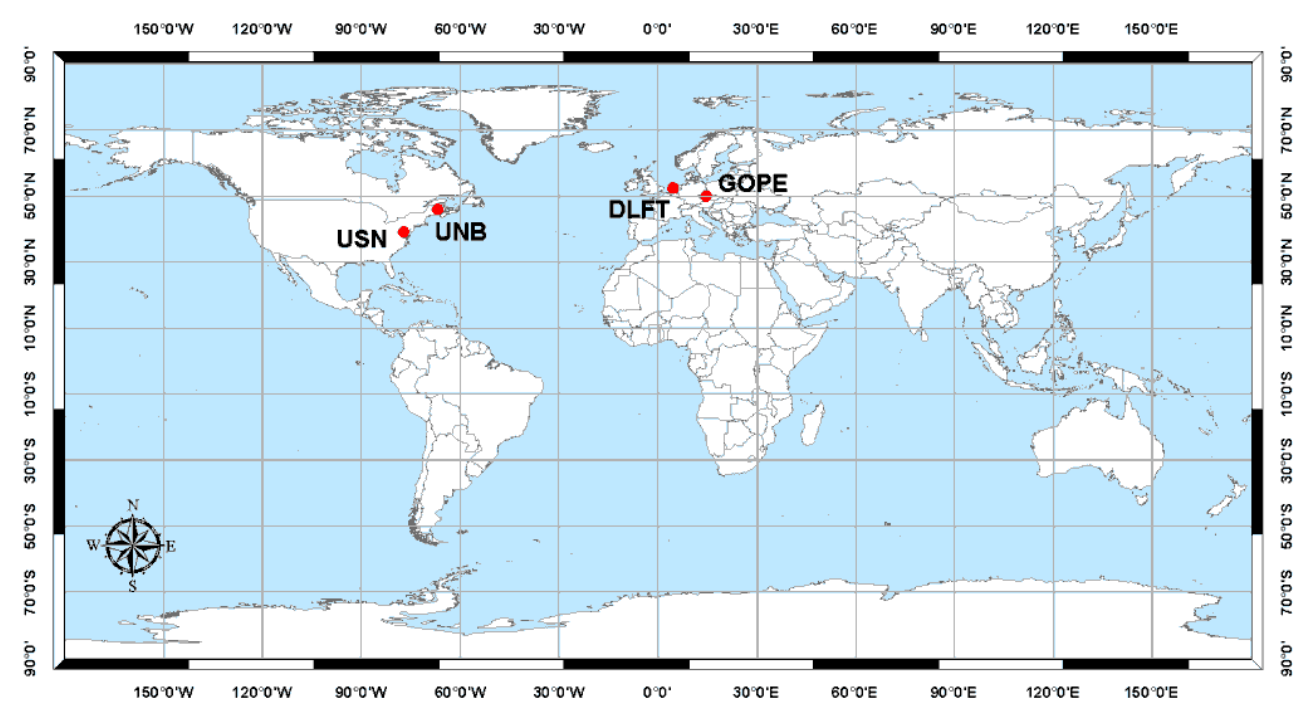

Figure 2.1 Analysis stations

The IGS global ionospheric maps (GIM) product is used to correct for the ionospheric delay (Schaer et al. 1998). In addition, The Hopfield tropospheric correction model along with the Vienna mapping function are used to account for the hydrostatic component of the tropospheric delay (Hopfield, 1972; Boehm and Schuh, 2004). CONGO network precise satellite orbit and 
clock corrections are used for both GPS and Galileo satellites. Only the results of stations DLFT (Europe) and UNB (North America) are presented in this paper. Similar results were obtained for other stations.

\subsubsection{UN-DIFFERENCED POSITIONING RESULTS}

The results of the un-differenced single frequency GPS PPP solution and the single-frequency GPS/Galileo PPP solution are obtained using two stochastic models, namely the sine function and the newly developed exponential function. Figure 2.2 shows the positioning results of the GPS-only PPP solution using the sine function as a representation of the observations stochastic model.
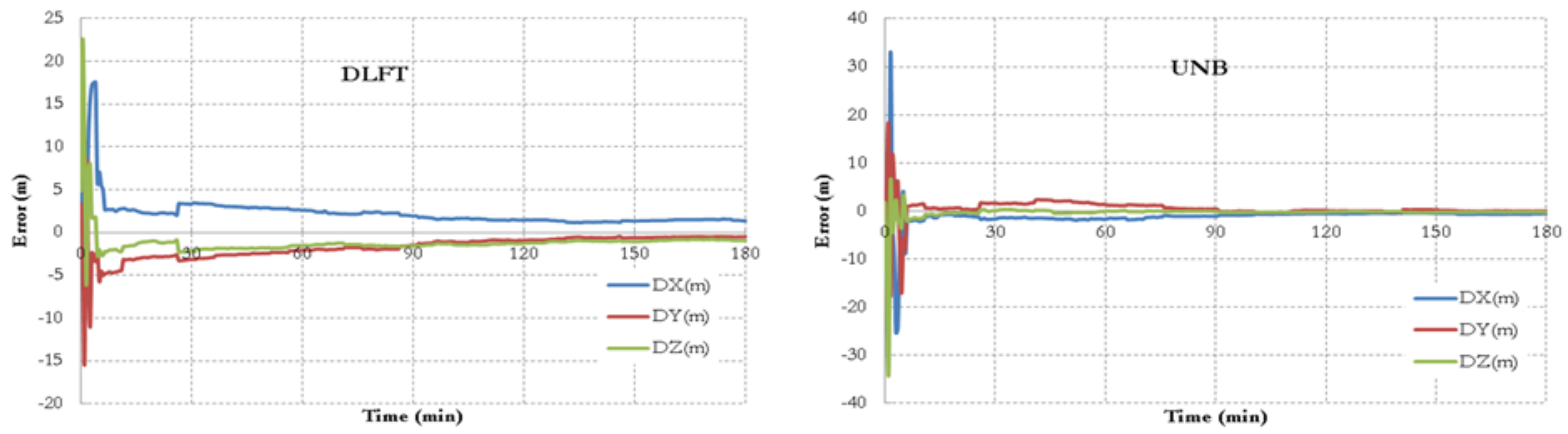

Figure 2.2 GPS PPP results using sine function stochastic model

As shown in Figure 2.2, the accuracy of the PPP solution with the GPS L1 signal is at the meter level. In contrast, when the newly developed exponential function is used, the single-frequency GPS PPP accuracy is improved to decimetre level (Figure 2.3).
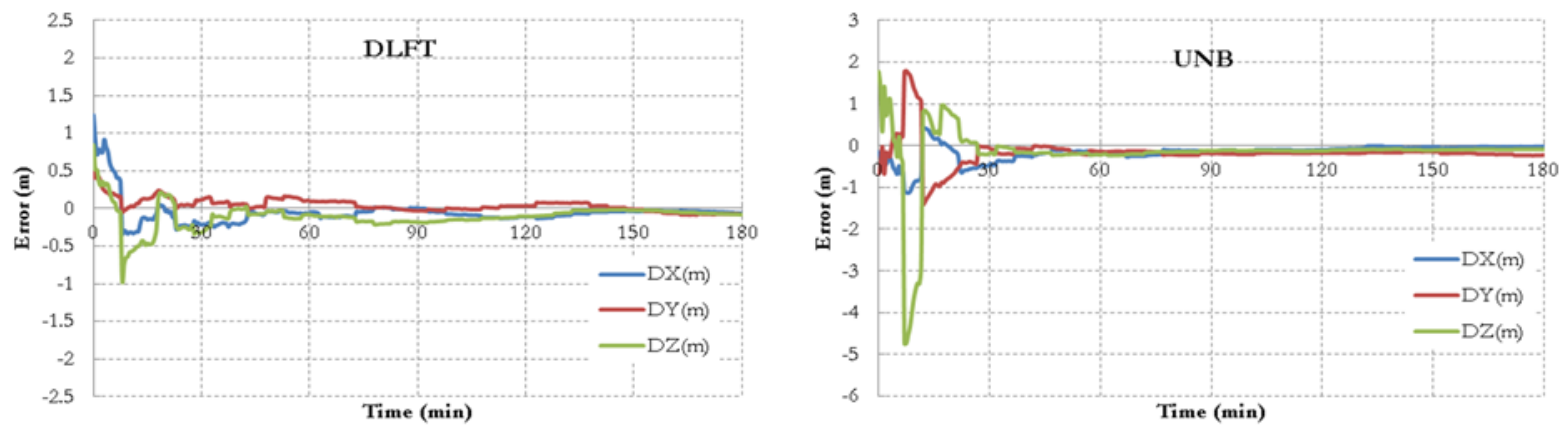

Figure 2.3 GPS PPP results using the newly developed stochastic model 
Figures 2.4 and 2.5 show the PPP results of the combined GPS/Galileo observations with the sine and exponential functions, respectively.
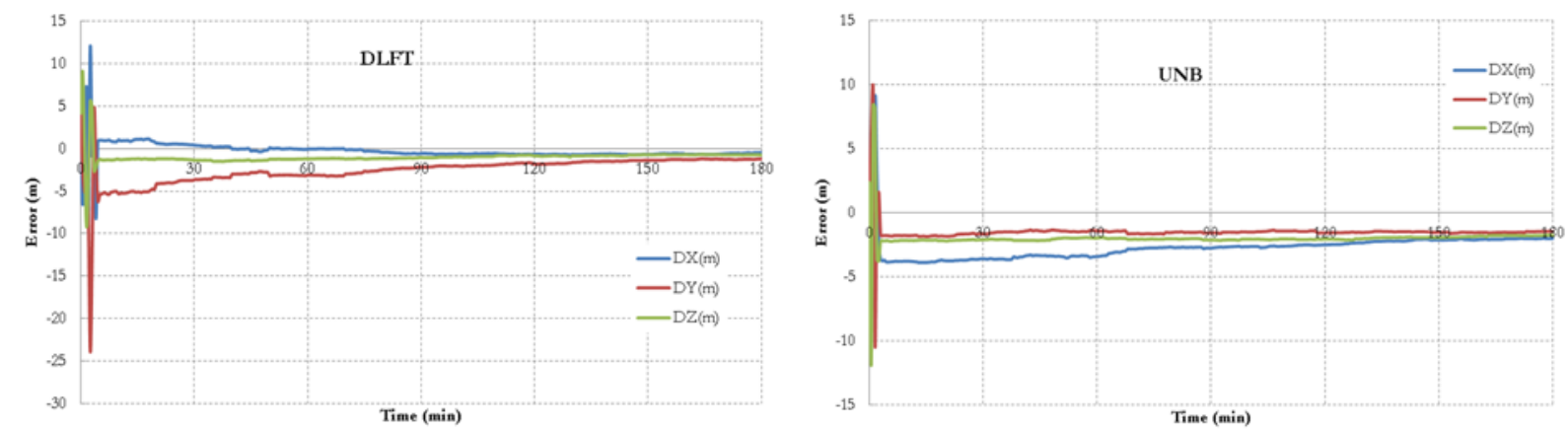

Figure 2.4 GPS/Galileo PPP results using empirical stochastic model

As can be seen in Figure 2.4, the results of the GPS/Galileo PPP with the sine function show decimetre-level accuracy; however the solution convergences to this accuracy level after about three hours. Figure 2.5 shows that, when the exponential function is used, the solution converges to decimetre-level after 100 minutes or less. This is considered significant improvement, especially with single-frequency observations.
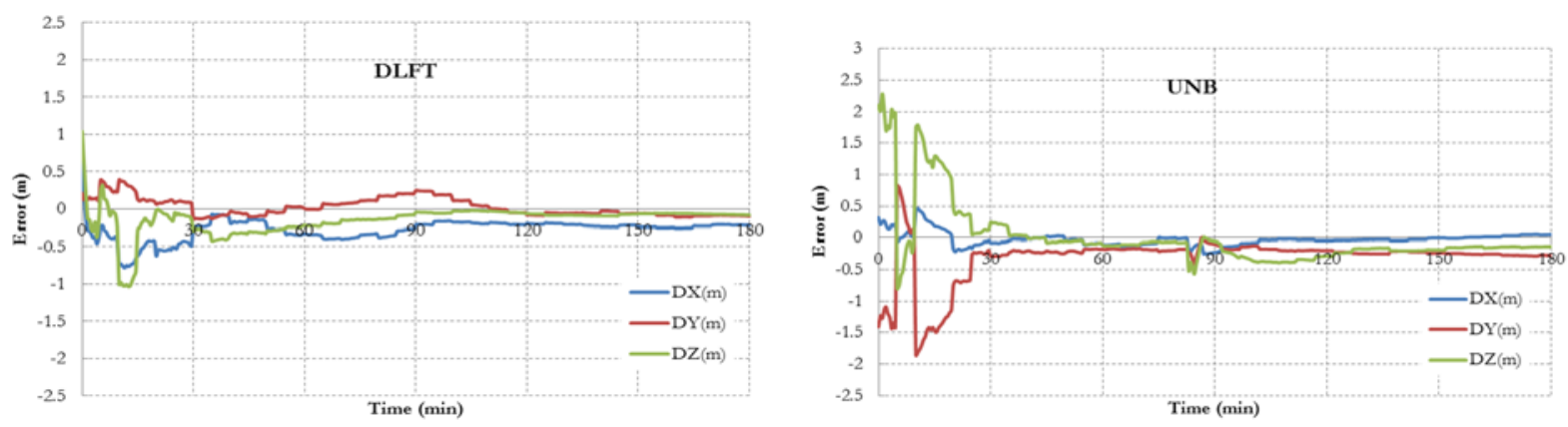

Figure 2.5 GPS/Galileo PPP results using the newly developed stochastic model

\subsubsection{BSSD POSITIONING RESULTS}

Similar to the un-differenced case, BSSD is considered for both GPS-only and GPS/Galileo with both the sine function and newly developed stochastic exponential function. A GPS satellite is considered as a reference when forming BSSD, as Afifi and El-Rabbany (2013) showed that 
better accuracy is obtained through this scenario. Figures 2.6 and 2.7 show the results of BSSD GPS PPP using both the sine and the exponential functions, respectively.
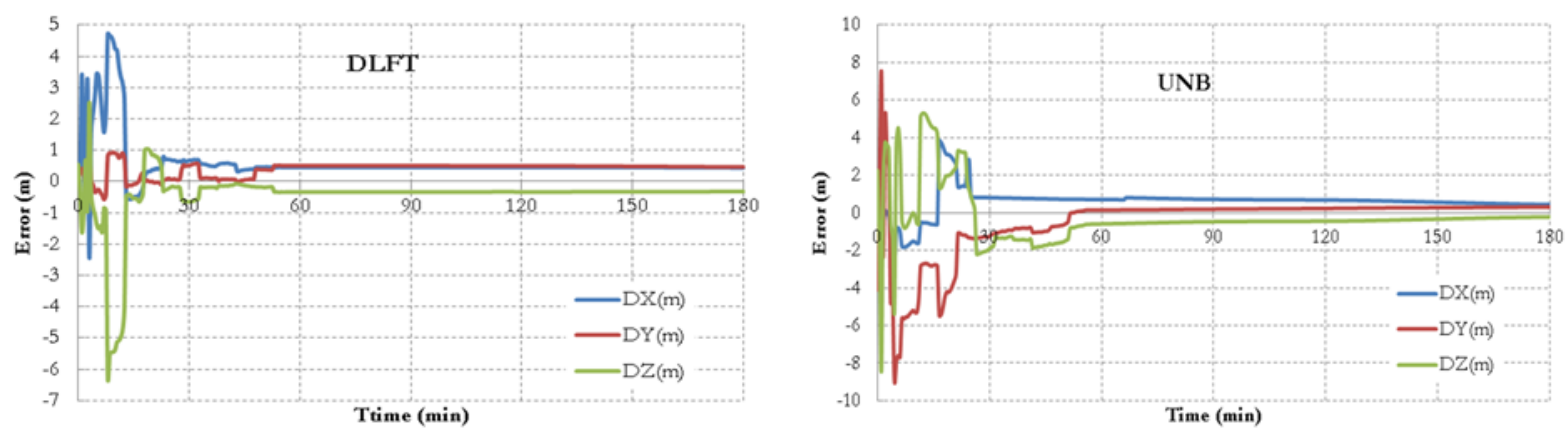

Figure 2.6 BSSD GPS PPP results using empirical sine function stochastic model

As shown in Figure 2.6, single-frequency GPS BSSD results with the sine function is converged to decimetre-level after about 90 minutes. The convergence time is reduced to 65 minutes or less when the exponential function is used (Figure 2.7).
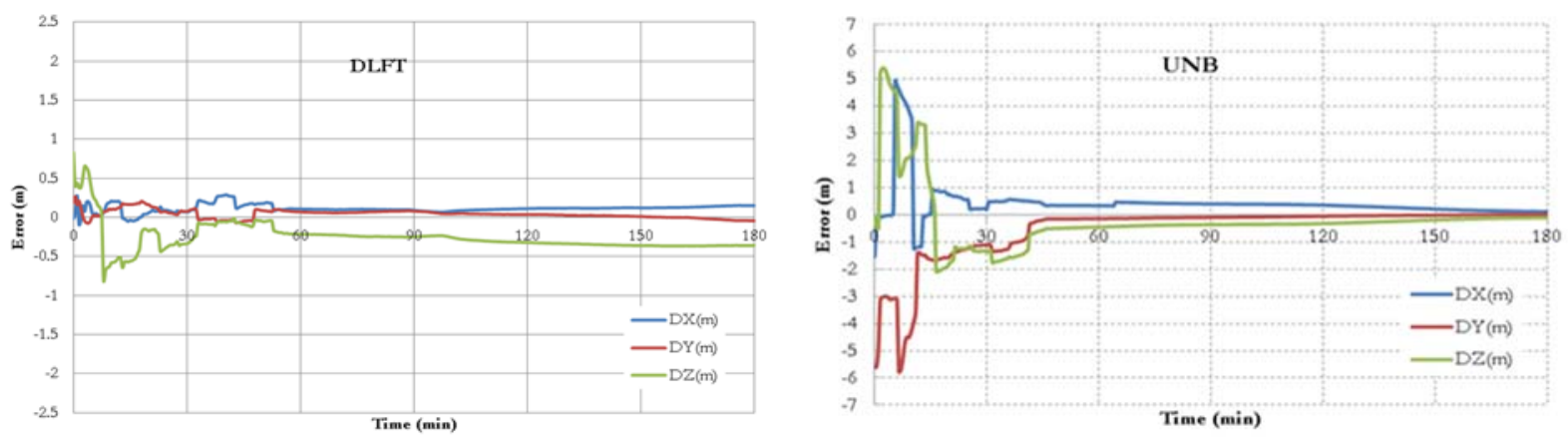

Figure 2.7 BSSD GPS PPP results using the newly developed stochastic model

Figures 2.8 and 2.9 show the PPP results of the combined BSSD GPS/Galileo observations with the sine and exponential functions, respectively. As can be seen, only slight improvement in the positioning accuracy and convergence time in comparison with the un-difference GPS/Galileo scenario. This suggests that both the un-differenced and BSSD GPS/Galileo PPP solutions are comparable. 

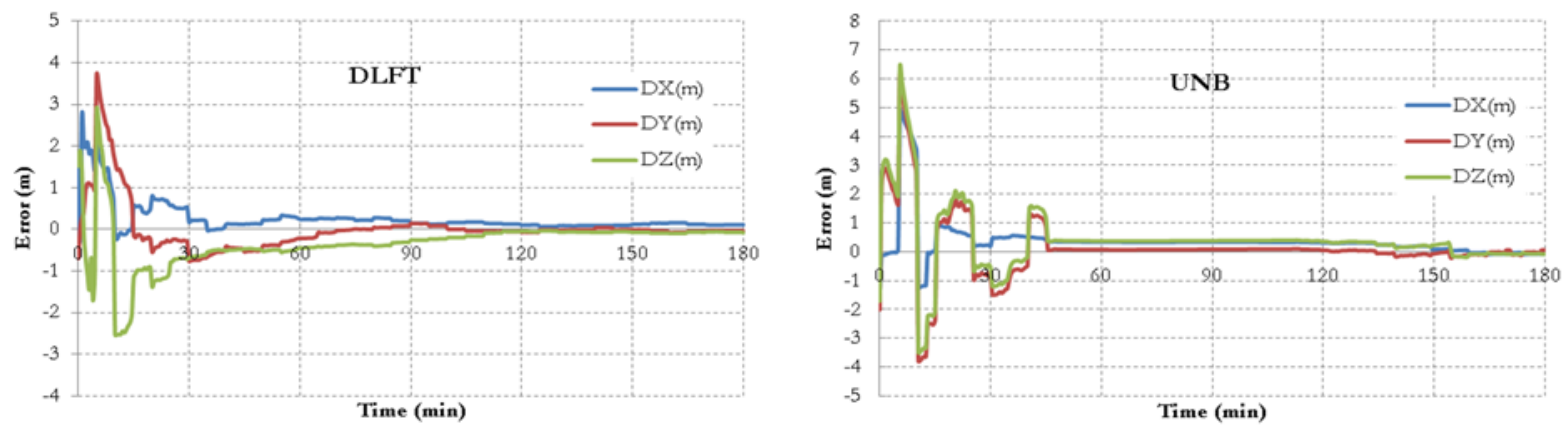

Figure 2.8 BSSD GPS/Galileo PPP results using empirical sine function stochastic model
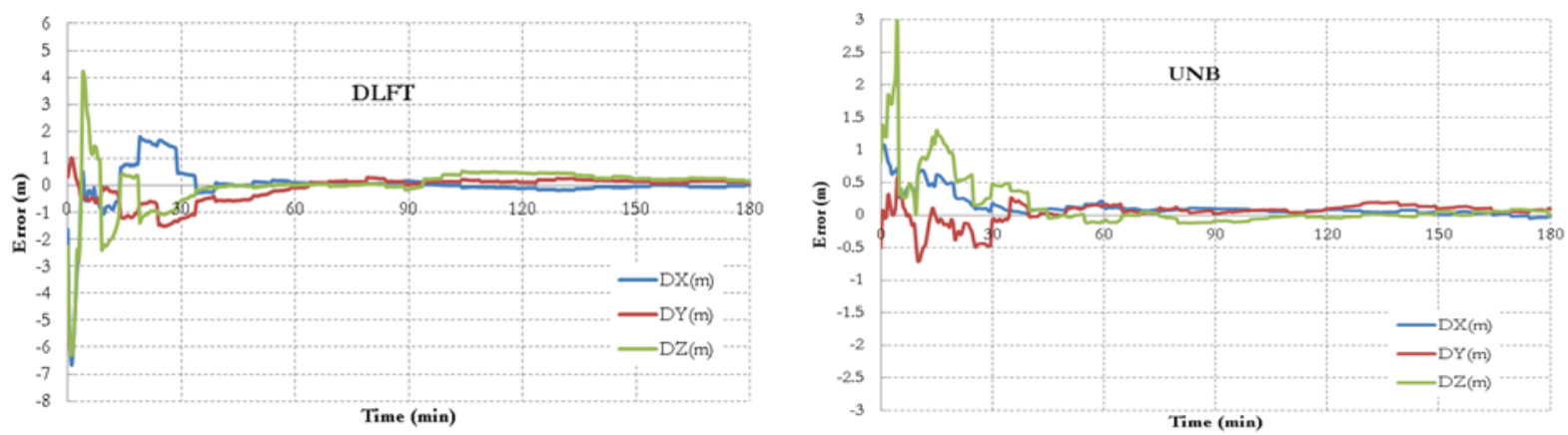

Figure 2.9 BSSD GPS/Galileo using the newly developed stochastic model

\subsection{CONCLUSIONS}

A new PPP model has been introduced in this paper, which combines GPS and Galileo system observations. The model considers both the un-differenced and BSSD modes. As well, a new stochastic model for Galileo E1 signal has been developed in this research. Three empirical functions have been considered, namely exponential, polynomial and rational functions. It has been found that the exponential function gives the best fit, based on regression analysis. It has been shown that a sub-decimetre positioning accuracy is attainable with single-frequency GPS/Galileo PPP when the newly developed exponential model is used. As well, the addition of Galileo improves the PPP solution convergence by about 30\% in comparison with GPS-only solution. 


\section{CHAPTER 3}

\section{AN IMPROVED MODEL FOR SINGLE FREQUENCY GPS/GALILEO PRECISE POINT POSITIONING}

This chapter provides a single-frequency PPP model using GPS and Galileo observations in BSSD mode. In this chapter, two scenarios are considered when forming the GPS and Galileo BSSD linear combination, namely a tight and a loose combination. In the first scenario, either a GPS or a Galileo satellite is selected as a reference for both GPS and Galileo observables. The second scenario considers two reference satellites: a GPS reference satellite for the GPS observables and a Galileo satellite for the Galileo observables. This chapter was published as:

Afifi, A., El-Rabbany, A. (2015). An improved model for single frequency GPS/Galileo precise point positioning. Positioning Journal, 6(2), 7-21, doi: 10.4236/pos.2015.62002.

Modifications to the original manuscript were made only for proper identification of sections, figures and tables, as well as to assure the uniformity of symbol and equation notation throughout this dissertation. 


\begin{abstract}
This paper introduces a new precise point positioning (PPP) model, which combines singlefrequency GPS/Galileo observations in between-satellite single-difference (BSSD) mode. In the absence of multipath, all receiver-related errors and biases are cancelled out when forming BSSD for a specific constellation. This leaves the satellite originating errors and atmospheric delays unmodelled. Combining GPS and Galileo observables introduces additional biases that have to be modelled, including the GPS to Galileo time offset (GGTO) and the inter-system bias. This paper models all PPP errors rigorously to improve the single-frequency GPS/Galileo PPP solution. GPSPace PPP software of Natural Resources Canada (NRCan) is modified to enable a GPS/Galileo PPP solution and to handle the newly introduced biases. A total of 12 data sets representing the GPS/Galileo measurements of six IGS-MEGX stations are processed to verify the newly developed PPP model. Precise satellite orbit and clock corrections from IGS-MEGX networks are used for both GPS and Galileo measurements. It is shown that sub-decimeter level accuracy is possible with single-frequency GPS/Galileo PPP. In addition, the PPP solution convergence time is improved from approximately 100 minutes for the un-differenced singlefrequency GPS/Galileo solution to approximately 65 minutes for the BSSD counterpart when a single reference satellite is used. Moreover, an improvement in the PPP solution convergence time of $35 \%$ and $15 \%$ is obtained when one and two reference satellites are used, respectively.
\end{abstract}

\title{
3.1. INTRODUCTION
}

The concept of precise point positioning (PPP) was first introduced by Zumberge et al. in 1997. Dual-frequency GPS PPP technique has been proven to be capable of providing positioning solution at the sub-decimeter level in static mode. This is achieved through rigorous modeling or estimation of all errors and biases. Both un-differenced and between-satellite single difference (BSSD) ionosphere-free linear combinations of carrier-phase and pseudorange measurements have been used (Kouba and Héroux, 2001; Colombo et al., 2004). More recently, Elsobeiey and El-Rabbany (2014) showed that a 50\% improvement in the PPP convergence time is possible with BSSD dual frequency ionosphere-free GPS, in comparison with the un-differenced counterpart. Unfortunately, dual-frequency GPS receivers may not provide a cost effective solution to many users. In addition, a drawback of a single GNSS system such as GPS is the availability of a sufficient number of visible satellites in urban areas. 
The Galileo satellite system offers additional visible satellites to the user, which is expected to enhance the satellite geometry and the overall PPP solution when combined with GPS (Hofmann-Wellenhof et al., 2008). As shown in Afifi and El-Rabbany (2013), combining GPS and Galileo observations in a PPP solution reduces the convergence time by up to $30 \%$ in comparison with the GPS-only PPP solution. Combining GPS and Galileo, however, comes at the expense of introducing additional biases to the observation mathematical models. These include the GPS to Galileo time offset, and Galileo satellite hardware delay. Recently, the European Space Agency (ESA) estimated the GPS to Galileo time offset (GGTO), which was found to be approximately $50 \mathrm{~ns}$, or equivalently $15 \mathrm{~m}$ range error (ESA, 2013).

Generally, combining multi-constellation observations in a PPP solution improves the positioning accuracy, especially when the system biases are calibrated, as shown in Melgard et al. (2013). Odijk and Teunissen (2013) showed that prior correction of the differential GPS/Galileo (GIOVE) inter-system biases significantly increases the success rate of instantaneous ambiguity resolution for short baselines. Likewise, Paziewski and Wielgosz (2013) showed that combining GPS/Galileo observables in a double-differenced carrier-phase and pseudorange technique improves the success rate of instantaneous ambiguity resolution in comparison with GPS-only solution. Unfortunately, however, their work was limited to differential positioning mode.

This paper introduces a new PPP model, which combines single frequency GPS and Galileo observables in BSSD mode. Precise corrections from the International GNSS Service multiGNSS experiment (IGS-MEGX) network are used to account for GPS and Galileo satellite orbit and clock errors (Montenbruck et al., 2014). As these products are presently referenced to the GPS time and since we use mixed GNSS receivers that also use GPS time as a reference, the GGTO is cancelled out in our model. The inter-system bias is either cancelled out through differencing the observations or is treated as an additional unknown parameter as detailed below. The ionospheric delay is largely corrected through the global ionosphere maps (GIM) model (Schaer et al. 1998). The hydrostatic component of the tropospheric zenith path delay is modelled through the Hopfield model, while the wet component is considered as an additional 
unknown parameter (Hofmann-Wellenhof et al., 2008). All remaining errors and biases are accounted for using existing models as shown in Kouba (2009). When forming BSSD, we consider three scenarios in the selection of the reference satellite. Either a GPS or a Galileo satellite is selected as a reference for both GPS and Galileo observables. Alternatively, two reference satellites are selected: a GPS reference satellite for the GPS observables and a Galileo satellite for the Galileo observables. The first approach is sometimes referred to as tight combination, while the latter is sometimes referred to as per constellation or loose combination (Paziewski and Wielgosz, 2014; Odijk and Teunissen, 2013). It is shown that the use of a single reference satellite provides a sub-decimeter level positioning accuracy and 35\% improvement in the convergence time, in comparison with the un-differenced single frequency GPS/Galileo solution. The use of two reference satellites, although provides comparable positioning accuracy, improves the solution convergence time by $15 \%$ only.

\subsection{UN-DIFFERENCED GPS/GALILEO MODEL}

GNSS observations are affected by random and systematic errors, which must be accounted for to ensure that precise positioning solution is obtained. The positioning accuracy of a PPP model depends on the ability to mitigate errors and biases. These errors can be categorized into three classes, namely satellite related errors, signal propagation related errors, and receiver/antenna associated errors. The main GNSS errors include the satellite/receiver clock errors, satellite/receiver hardware delays, ionospheric and tropospheric delays, and multipath (ElRabbany, 2006).

In addition to the above errors and biases, combining GPS and Galileo observations in a PPP model introduces additional errors such as GGTO due to the fact that each system uses a different time frame. The GPS system uses the GPS time system, which is referenced to coordinated universal time (UTC) as maintained by the US Naval Observatory (USNO). On the other hand, the Galileo satellite system has its own time frame, namely the Galileo system time (GST), which is a continuous atomic time scale with a nominal constant offset with respect to the international atomic time (TAI) (Hofmann-Wellenhof et al., 2008). Taking the above errors and biases into consideration and assuming that the observations are taking simultaneously from a 
mixed GNSS receiver, which uses GPS time as a reference, the GPS and Galileo observation equations can be written as:

$$
\begin{gathered}
P_{G}\left(t_{G}\right)=\rho_{G}\left(t_{G}, t_{G}-\tau_{G}\right)+c\left[d_{r G}\left(t_{G}\right)+d_{G}^{S}\left(t_{G}-\tau_{G}\right)\right]+c\left[d t_{r G}\left(t_{G}\right)-d t_{G}^{S}\left(t_{G}-\tau_{G}\right)\right]+T_{G}+I_{G}+\varepsilon_{P G} \\
P_{E}\left(t_{G}\right)=\rho_{E}\left(t_{G}, t_{G}-\tau_{E}\right)+c\left[d_{r E}\left(t_{G}\right)+d_{E}^{S}\left(t_{G}-\tau_{E}\right)\right]+c\left[d t_{r G}\left(t_{G}\right)-G G T O-d t_{E}^{S}\left(t_{G}-\tau_{E}\right)\right]+T_{E}+I_{E}+\varepsilon_{P E} \\
\Phi_{G}\left(t_{G}\right)=\rho_{G}\left(t_{G}, t_{G}-\tau_{G}\right)+c\left[\delta_{r G}\left(t_{G}\right)+\delta_{G}^{S}\left(t_{G}-\tau_{G}\right)\right]+c\left[d t_{r G}\left(t_{G}\right)-d t_{G}^{S}\left(t_{G}-\tau_{G}\right)\right]+ \\
T_{G}-I_{G}+\lambda_{G}\left[N+\varphi_{r}\left(t_{0}\right)-\varphi^{S}\left(t_{0}\right)\right]_{G}+\varepsilon_{\Phi G} \\
\Phi_{E}\left(t_{G}\right)=\rho_{E}\left(t_{G}, t_{G}-\tau_{E}\right)+c\left[\delta_{r E}\left(t_{G}\right)+\delta_{E}^{S}\left(t_{G}-\tau_{E}\right)\right]+c\left[d t_{r G}\left(t_{G}\right)-G G T O-d t_{E}^{S}\left(t_{G}-\tau_{E}\right)\right]+ \\
T_{E}-I_{E}+\lambda_{E}\left[N+\varphi_{r}\left(t_{0}\right)-\varphi^{S}\left(t_{0}\right)\right]_{E}+\varepsilon_{\Phi E}
\end{gathered}
$$

where, the subscript $G$ refers to the GPS satellite system and the subscript $E$ refers to the Galileo satellite system; $t_{G}$ is the true signal reception time; $\tau_{G}$ and $\tau_{E}$ are signal propagation times for both GPS and Galileo, respectively; $P_{G}$ and $P_{E}$ are the GPS and Galileo pseudorange measurements, respectively; $\Phi_{G}$ and $\Phi_{E}$ are the GPS and Galileo carrier-phase measurements, respectively; $\rho_{G}\left(t_{G}, t_{G}-\tau_{G}\right)$ and $\rho_{E}\left(t_{G}, t_{G^{-}} \tau_{E}\right)$ are the GPS and Galileo geometric ranges from the receiver at reception time $t_{G}$ to the satellite at transmission times $\left(t_{G}-\tau_{G}\right)$ and $\left(t_{G}-\tau_{E}\right)$, respectively; $d t_{r G}\left(t_{G}\right)$ is the receiver clock error at reception time $t_{G} ; d t_{G} s\left(t_{G}-\tau_{G}\right)$ and $d t_{E} s\left(t_{G}-\tau_{E}\right)$ are the GPS and Galileo satellite clock errors at transmission times $\left(t_{G}-\tau_{G}\right)$ and $\left(t_{G}-\tau_{E}\right)$, respectively; $d_{r G}\left(t_{G}\right)$ and $d_{r E}\left(t_{G}\right)$ are frequency-dependent code hardware delays in the receiver at reception time $t_{G}$ for GPS and Galileo, respectively; $d_{G} s\left(t_{G}-\tau_{G}\right)$ and $d_{E}^{s}\left(t_{G}-\tau_{E}\right)$ are frequency-dependent code hardware delays in the satellites at transmission time $\left(t_{G}-\tau_{G}\right)$ and $\left(t_{G}-\tau_{E}\right)$ for GPS and Galileo, respectively; $\delta_{r G}\left(t_{G}\right)$ and $\delta_{G}^{s}\left(t_{G-} \tau_{G}\right)$ are frequency-dependent carrier phase hardware delays in the receiver at reception time $t_{G}$ for GPS and Galileo, respectively; $\delta_{r E}\left(t_{G}\right)$ and $\delta_{E}^{s}\left(t_{G}-\tau_{E}\right)$ are frequencydependent carrier phase hardware delays in the satellites at transmission time $\left(t_{G}-\tau_{G}\right)$ and $\left(t_{G}-\tau_{E}\right)$ for GPS and Galileo, respectively; $T$ is the tropospheric delay; $I$ is the ionospheric delay; $\lambda_{G}$ and $\lambda_{E}$ are the wavelengths of carrier frequencies for GPS and Galileo signals, respectively; $\Phi_{\mathrm{r}}\left(t_{0}\right)$, $\Phi^{s}\left(t_{0}\right)$ are frequency-dependent initial fractional phases in the receiver and satellite channels, respectively; to is the receiver (or satellite) initial time; $N_{G}$ and $N_{E}$ are the integer numbers of cycles for GPS and Galileo carrier phase measurements, respectively; GGTO is the GPS to Galileo time offset; $\mathrm{c}$ is the speed of light in vacuum; and $\varepsilon_{\mathrm{P}}, \varepsilon_{\Phi}$ are the relevant noise and unmodeled errors. 
As indicated earlier, precise orbit and satellite clock corrections of IGS-MGEX network are used for both GPS and Galileo observations. Clock corrections from the two networks are referred to the GPS time. In addition, they include the ionosphere-free linear combination of the satellite hardware delays of L1/L2 P(Y) code for GPS and the ionosphere-free linear combination of the satellite hardware delays of E1/E5a pilot code for Galileo (Steigenberger et al., 2014). As such, using Equations 3.1-3.4 and dropping the time arguments, the L1/E1 single frequency code and carrier phase observation equations take the form:

$$
\begin{aligned}
& P_{P 1}=\rho_{G}+\tilde{d} t_{r G}-c\left[d t_{\operatorname{prec}(G)}^{s}+\beta_{G} D C B_{P 1-P 2}^{s}\right]+T_{G}+I_{P 1}+\varepsilon_{P G} \\
& P_{C 1}=\rho_{G}+\tilde{d} t_{r G}-c\left[d t_{p r e c(G)}^{s}+\beta_{G} D C B_{P I-P 2}^{s}+D C B_{P I-C 1}^{s}\right]+T_{G}+I_{1}+\varepsilon_{P G} \\
& P_{E 1}=\rho_{E}+\tilde{d} t_{r G}-c\left[d t_{p r e c(E)}^{s}+\beta_{E} D C B_{E I-E S a}^{s}\right]+T_{E}+I_{1}+I S B+\varepsilon_{P E} \\
& \Phi_{L 1}=\rho_{G}+\tilde{d} t_{r G}-c\left[d t_{p r e c(G)}^{s}+\beta_{G} D C B_{P l-P 2}^{s}\right]+T_{G}-I_{1}+\tilde{N}_{L 1}+\varepsilon_{\Phi L 1} \\
& \Phi_{E 1}=\rho_{E}+\tilde{d} t_{r G}-c\left[d t_{p r e c(E)}^{s}+\beta_{E} D C B_{E l-E 5 a}^{s}\right]+T_{E}-I_{1}+I S B+\tilde{N}_{E 1}+\varepsilon_{\Phi E 1}
\end{aligned}
$$

where $d t^{s} \operatorname{prec}_{(G)}$ and $d t^{s} \operatorname{prec}(E)$ are the precise satellites clock corrections for both GPS and Galileo

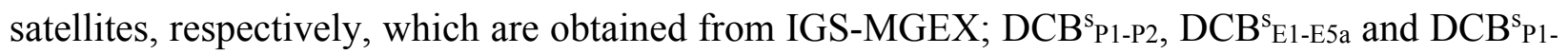
$\mathrm{C} 1$ are the satellite differential code biases for GPS P1/P2, P1/C1 and Galileo E1/E5a signals, respectively, which are obtained from IGS; $\beta_{G}$ and $\beta_{E}$ are the ionosphere-free linear combination coefficients, which equal 1.546 and 1.261 for GPS and Galileo, respectively; $\tilde{d} t_{r G}$ represents the combined effect of the receiver clock error and the GPS receiver code hardware delay, i.e., $\tilde{d} t_{r G}=c\left[d_{r P 1}+d t_{r G}\right]$ when P-code is used and $\tilde{d} t_{r G}=c\left[d_{r C l}+d t_{r G}\right]$ when C/A-code is used; $I S B$ is the inter-system bias, which equals $c\left[d_{r P 1}-d_{r E I}\right]$ when P-code is used and equals $c\left[d_{r C l}-d_{r E I}\right]$ when C/A-code is used; $\tilde{N}$ is the ambiguity parameter lumped to the satellite and receiver hardware delays, i.e.,

$$
\begin{aligned}
& \tilde{N}_{L I}=\lambda\left[N+\varphi_{r}-\varphi^{S}\right]_{G}+c\left[\delta_{r L 1}+\delta_{L I}^{s}\right]-c\left[d_{r P 1}+d_{P I}^{s}\right] \\
& \tilde{N}_{E I}=\lambda\left[N+\varphi_{r}-\varphi^{S}\right]_{E}+c\left[\delta_{r E 1}+\delta_{E I}^{s}\right]-c\left[d_{r E 1}+d_{E I}^{s}\right]
\end{aligned}
$$

It should be pointed out that in both of our GPS-only and GPS/Galileo PPP models, the GPS receiver hardware delay is lumped to the receiver clock error as explained above. This strategy 
maintains the consistency of the estimated receiver clock error for both of the GPS-only and the GPS/Galileo PPP solutions (Afifi and El-Rabbany, 2014). It should also be mentioned that some of our data sets contain the C/A-code pseudorange rather than the P-code pseudorange. In this case, Equation 3.6 should replace Equation 3.5 for the GPS code measurements. In addition, the receiver clock error would be lumped to the receiver hardware delay of the C/A code. Furthermore, the $I S B$ would equal the difference in the receiver hardware delays of the $\mathrm{C} / \mathrm{A}$ code and E1 code, scaled by the speed of light.

Equations 3.5 to 3.9 can be simplified for the pseudorange and carrier phase observables after applying the corrections for the satellite clock errors, the hydrostatic component of the tropospheric zenith path delay, the correction to the ionospheric delay, the satellite differential code biases, and the other remaining biases. As stated earlier, the global ionosphere maps (GIM) are used to account for the ionospheric delay (Schaer et al. 1998). Generally, GIM ionospheric model was found superior to other global models such as the Klobuchar model (Chen and Gao, 2005; Abd El-Rahman and El-Rabbany, 2013). The Hopfield tropospheric correction model is used, along with Vienna mapping function, to account for the hydrostatic component of the tropospheric delay (Hopfield, 1972; Boehm and Schuh, 2004). All other remaining biases are modeled using existing models, including the effects of ocean loading (Bos and Scherneck, 2014; IERS, 2010), Earth tide (Kouba, 2009), carrier-phase windup (Leick, 2004; Wu et al., 1993), Sagnac (Kaplan and Hegarty, 2006), relativity (Hofmann-Wellenhof et al., 2008), and satellite and receiver antenna phase-center variations (Dow et al. 2009). Applying these corrections and considering P-code observations only lead to:

$$
\begin{aligned}
& \rho_{G}+\tilde{d} t_{r G}+m_{f} z p d_{w}+\tilde{\varepsilon}_{P G}-\tilde{P}_{P 1}=0 \\
& \rho_{E}+\tilde{d} t_{r G}+m_{f} z p d_{w}+I S B+\tilde{\varepsilon}_{P E}-\tilde{P}_{E 1}=0 \\
& \rho_{G}+\tilde{d} t_{r G}+m_{f} z p d_{w}+\tilde{N}_{L 1}+\tilde{\varepsilon}_{\Phi L 1}-\tilde{\Phi}_{L 1}=0 \\
& \rho_{E}+\tilde{d} t_{r G}+m_{f} z p d_{w}+I S B+\tilde{N}_{E 1}+\tilde{\varepsilon}_{\Phi E 1}-\tilde{\Phi}_{E 1}=0
\end{aligned}
$$

where $\tilde{P}$ and $\widetilde{\Phi}$ are the corrected carrier phase and pseudorange observables; $z p d_{w}$ is the wet component of the tropospheric zenith path; $m_{f}$ is the troposphere mapping function, respectively; $\tilde{\varepsilon}_{P G}, \tilde{\varepsilon}_{P E}, \tilde{\varepsilon}_{\Phi L 1}$ and $\tilde{\varepsilon}_{\Phi E l}$ are the noise terms. 


\subsection{BSSD GPS/GALILEO COMBINATION MODEL}

Differencing the observations between satellites cancels out most receiver-related errors, including receiver clock error, receiver hardware delay for the same constellation, and non-zero initial phase bias (El-Rabbany, 2006). As the L1 and E1 frequencies are the same, we can select a single satellite, either a GPS or a Galileo as a reference when forming the BSSD. This means that the observations of all other GPS and Galileo satellites are differenced with respect to the observations of that satellite. As indicated earlier, this approach is sometimes referred to as tight combination. Alternatively, two reference satellites can be used, i.e., per constellation BSSD. The former approach produces two additional BSSD equations in comparison with the second approach, one for code and another for carrier-phase observables. However, the ISB is cancelled out when the per-constellation approach is used. When a GPS satellite is used as a reference in a tight combination, we obtain:

$\nabla \rho_{G, G}^{i j}+m_{f}^{i j} z p d_{w}+\nabla \tilde{\mathcal{E}}_{P G}^{i j}-\nabla \tilde{P}_{P 1}^{i j}=0$

$\nabla \rho_{E, G}^{i k}+m_{f}^{i k} z p d_{w}+I S B+\nabla \tilde{\varepsilon}_{P E}^{i k}-\nabla \tilde{P}_{E 1 G}^{i k}=0$

$\nabla \rho_{G, G}^{i j}+m_{f}^{i j} z p d_{w}+\tilde{N}_{L 1}^{i j}+\nabla \tilde{\varepsilon}_{\Phi L 1}^{i j}-\nabla \tilde{\Phi}_{L 1}^{i j}=0$

$\nabla \rho_{E, G}^{i k}+m_{f}^{i k} z p d_{w}+I S B+\tilde{N}_{E 1}^{i k}+\nabla \tilde{\varepsilon}_{\Phi E 1}^{i k}-\nabla \tilde{\Phi}_{E 1 G}^{i k}=0$

where $\nabla$ refers to the BSSD operator; $\tilde{N}_{L 1}^{i j}$ and $\tilde{N}_{E 1}^{i k}$ are the BSSD non-integer the ambiguity parameters lumped to the receiver and satellite hardware delays as shown in Equations 3.20 and 3.21.

$\tilde{N}_{L 1}^{i j}=\lambda\left[N_{G}^{j}-N_{G}^{i}\right]-\lambda\left[\varphi_{G}^{j}-\varphi_{G}^{i}\right]+c\left[\delta_{L 1}^{j}-\delta_{L 1}^{i}\right]-c\left[d_{P 1}^{j}-d_{P I}^{i}\right]$

$\tilde{N}_{E 1}^{i k}=\lambda\left[N_{E}^{k}-N_{G}^{i}\right]-\lambda\left[\varphi_{G}^{i}-\varphi_{E}^{k}\right]+c\left[\delta_{r E 1}+\delta_{E 1}^{k}\right]-c\left[\delta_{r L l}+\delta_{L I}^{i}\right]-c\left[d_{r E l}+d_{E l}^{k}\right]+c\left[d_{r P l}+d_{P I}^{i}\right]$

If, however, a Galileo satellite is used as a reference in a tight combination, we obtain the following set of BSSD equations:

$$
\begin{aligned}
& \nabla \rho_{G, E}^{l j}+m_{f}^{l j} z p d_{w}-I S B+\nabla \tilde{\varepsilon}_{P G}^{l j}-\nabla \tilde{P}_{P 1 E}^{l j}=0 \\
& \nabla \rho_{E, E}^{l k}+m_{f}^{l k} z p d_{w}+\nabla \tilde{\varepsilon}_{P E}^{l k}-\nabla \tilde{P}_{E 1}^{l k}=0
\end{aligned}
$$


$\nabla \rho_{G, E}^{l j}+m_{f}^{l j} z p d_{w}-I S B+\tilde{N}_{L 1}^{l j}+\nabla \tilde{\varepsilon}_{\Phi L 1}^{l j}-\nabla \tilde{\Phi}_{L 1 E}^{l j}=0$

$\nabla \rho_{E, E}^{l k}+m_{f}^{l k} z p d_{w}+\tilde{N}_{E 1}^{l k}+\nabla \tilde{\varepsilon}_{\Phi E 1}^{l k}-\nabla \tilde{\Phi}_{E 1}^{l k}=0$

where, $\tilde{N}_{L 1}^{l j}$ and $\tilde{N}_{E 1}^{l k}$ are the BSSD non-integer ambiguity parameters lumped to the receiver and satellite hardware delays as shown in Equations 3.26 and 3.27.

$\tilde{N}_{L 1}^{j j}=\lambda\left[N_{G}^{j}-N_{E}^{l}\right]-\lambda\left[\varphi_{E}^{l}-\varphi_{G}^{j}\right]+c\left[\delta_{r L 1}+\delta_{L 1}^{j}\right]-c\left[\delta_{r E I}+\delta_{E I}^{l}\right]-c\left[d_{r P I}+d_{P I}^{j}\right]+c\left[d_{r E l}+d_{E l}^{l}\right]$

$\tilde{N}_{E 1}^{l k}=\lambda\left[N_{E}^{k}-N_{E}^{l}\right]-\lambda\left[\varphi_{E}^{k}-\varphi_{E}^{l}\right]+c\left[\delta_{E I}^{k}-\delta_{E I}^{l}\right]-c\left[d_{E I}^{k}-d_{E I}^{l}\right]$

Finally, the per-constellation BSSD equations take the form:

$\nabla \rho_{G, G}^{i j}+m_{f}^{i j} z p d_{w}+\nabla \tilde{\varepsilon}_{P G}^{i j}-\nabla \tilde{P}_{P 1}^{i j}=0$

$\nabla \rho_{E, E}^{l k}+m_{f}^{l k} z p d_{w}+\nabla \tilde{\varepsilon}_{P E}^{l k}-\nabla \tilde{P}_{E 1}^{l k}=0$

$\nabla \rho_{G, G}^{i j}+m_{f}^{i j} z p d_{w}+\tilde{N}_{L 1}^{i j}+\nabla \tilde{\varepsilon}_{\Phi L 1}^{i j}-\nabla \tilde{\Phi}_{L 1}^{i j}=0$

$\nabla \rho_{E, E}^{l k}+m_{f}^{l k} z p d_{w}+\tilde{N}_{E 1}^{l k}+\nabla \tilde{\varepsilon}_{\Phi E 1}^{l k}-\nabla \tilde{\Phi}_{E 1}^{l k}=0$

where, $\tilde{N}_{L 1}^{i j}$ and $\tilde{N}_{E 1}^{l k}$ are the BSSD non-integer ambiguity parameters lumped to the receiver and satellite hardware delays as shown in Equations 3.32 and 3.33.

$\tilde{N}_{L 1}^{i j}=\lambda\left[N_{G}^{j}-N_{G}^{i}\right]-\lambda\left[\varphi_{G}^{j}-\varphi_{G}^{i}\right]+c\left[\delta_{L 1}^{j}-\delta_{L 1}^{i}\right]-c\left[d_{P I}^{j}-d_{P I}^{i}\right]$
$\tilde{N}_{E 1}^{l k}=\lambda\left[N_{E}^{k}-N_{E}^{l}\right]-\lambda\left[\varphi_{E}^{k}-\varphi_{E}^{l}\right]+c\left[\delta_{E I}^{k}-\delta_{E I}^{l}\right]-c\left[d_{E I}^{k}-d_{E I}^{l}\right]$

It should be noticed from the above equations that the modified receiver clock error (i.e., the common term $\tilde{d} t_{r G}$ ) and the initial phase bias cancel out when forming BSSD with one satellite selected as a reference (i.e., tight combination). However, when forming per constellation BSSD, the modified receiver clock error, the inter-system bias, and the initial phase bias are all cancelled out.

\subsection{SEQUENTIAL LEAST SQUARES ESTIMATION}

The sequential least-squares estimation technique is used to obtain the best estimates, in the least squares sense, of the unknown parameters. The noise terms in the above observations equations are modeled stochastically using an exponential function, as described in Afifi and El-Rabbany 
(2013). They showed that, in comparison with existing stochastic models such as the general sine function, the use of the exponential model improves the PPP solution precision and convergence time. The general linearized form for the above observations equations around the initial (approximate) vector $\boldsymbol{u}^{0}$ and observables $l$ can be written in a compact form as:

$f(u, l)=A \Delta u-w-r=0$

where $\boldsymbol{u}$ is the vector of unknown parameters; $\boldsymbol{A}$ is the design matrix, which includes the partial derivatives of the observation equations with respect to the unknown parameters $\boldsymbol{u} ; \boldsymbol{\Delta} \boldsymbol{u}$ is the unknown vector of corrections to the approximate parameters $\boldsymbol{u}^{0}$, i.e., $\boldsymbol{u}=\boldsymbol{u}^{0}+\boldsymbol{\Delta} \boldsymbol{u} ; \boldsymbol{w}$ is the misclosure vector and $\boldsymbol{r}$ is the vector of residuals. The sequential least-squares solution for the unknown parameters $\boldsymbol{\Delta} \boldsymbol{u}_{\boldsymbol{i}}$ at an epoch $i$ can be obtained from (Vanicek and Krakiwsky, 1986):

$$
\begin{aligned}
& \Delta \boldsymbol{u}_{i}=\Delta \boldsymbol{u}_{i-1}+\boldsymbol{M}_{i-1}^{-1} \boldsymbol{A}_{i}^{T}\left(\boldsymbol{C}_{l_{i}}+\boldsymbol{A}_{i} \boldsymbol{M}_{i-1}^{-1} \boldsymbol{A}_{i}^{T}\right)^{-1}\left[\boldsymbol{w}_{i}-\boldsymbol{A}_{i} \Delta \boldsymbol{u}_{i-1}\right] \\
& \boldsymbol{M}_{i}^{-1}=\boldsymbol{M}_{i-1}^{-1}-\boldsymbol{M}_{i-1}^{-1} \boldsymbol{A}_{i}^{T}\left(\boldsymbol{C}_{l_{i}}+\boldsymbol{A}_{i} \boldsymbol{M}_{i-1}^{-1} \boldsymbol{A}^{T}\right)^{-1} \boldsymbol{A}_{i} \boldsymbol{M}_{i-1}^{-1} \\
& \boldsymbol{C}_{\Delta u_{i}}=\boldsymbol{M}_{i}^{-1}=\boldsymbol{M}_{i-1}^{-1}-\boldsymbol{M}_{i-1}^{-1} \boldsymbol{A}_{i}^{T}\left(\boldsymbol{C}_{l_{i}}+\boldsymbol{A}_{i} \boldsymbol{M}_{i-1}^{-1} \boldsymbol{A}^{T}\right)^{-1} \boldsymbol{A}_{i} \boldsymbol{M}_{i-1}^{-1}
\end{aligned}
$$

where $\boldsymbol{\Delta} \boldsymbol{u}_{i-1}$ is the least-squares solution for the estimated parameters at epoch $i-1 ; \boldsymbol{M}$ is the matrix of the normal equations; $\boldsymbol{C}_{l}$ and $\boldsymbol{C}_{\Delta u}$ are the covariance matrices of the observations and unknown parameters, respectively. It should be pointed out that the usual batch least-squares adjustment should be used in the first epoch, i.e., for $i=1$. The batch solution for the estimated parameters and the inverse of the normal equation matrix are given respectively by (Vanicek and Krakiwsky, 1986):

$$
\begin{aligned}
& \Delta \boldsymbol{u}_{1}=\left[\boldsymbol{C}_{x^{0}}^{-1}+\boldsymbol{A}_{l}^{T} \boldsymbol{C}_{l_{1}}^{-1} \boldsymbol{A}_{l}\right]^{-1} \boldsymbol{A}_{l}^{T} \boldsymbol{C}_{l_{1}}^{-1} \boldsymbol{w}_{1} \\
& \boldsymbol{M}_{1}^{-1}=\left[\boldsymbol{C}_{x^{0}}^{-1}+\boldsymbol{A}_{l}^{T} \boldsymbol{C}_{l_{1}}^{-1} \boldsymbol{A}_{l}\right]^{-1}
\end{aligned}
$$

where $C_{x}^{0}$ is a priori covariance matrix for the approximate values of the unknown parameters.

Under the assumption that the observations are uncorrelated and the errors are normally distributed with zero mean, the covariance matrix of the un-differenced observations takes the form of a diagonal matrix. The elements along the diagonal line represent the variances of the 
code and carrier phase measurements. In our solution, we consider that the ration between the standard deviation of the code and carrier-phase measurements to be 100. When forming BSSD, however, the differenced observations become mathematically correlated. This leads to a fully populated covariance matrix at a particular epoch.

Considering the un-differenced mode, the matrix $\boldsymbol{A}$ and the vector $\boldsymbol{\Delta} \boldsymbol{u}$ at a particular epoch are given by:

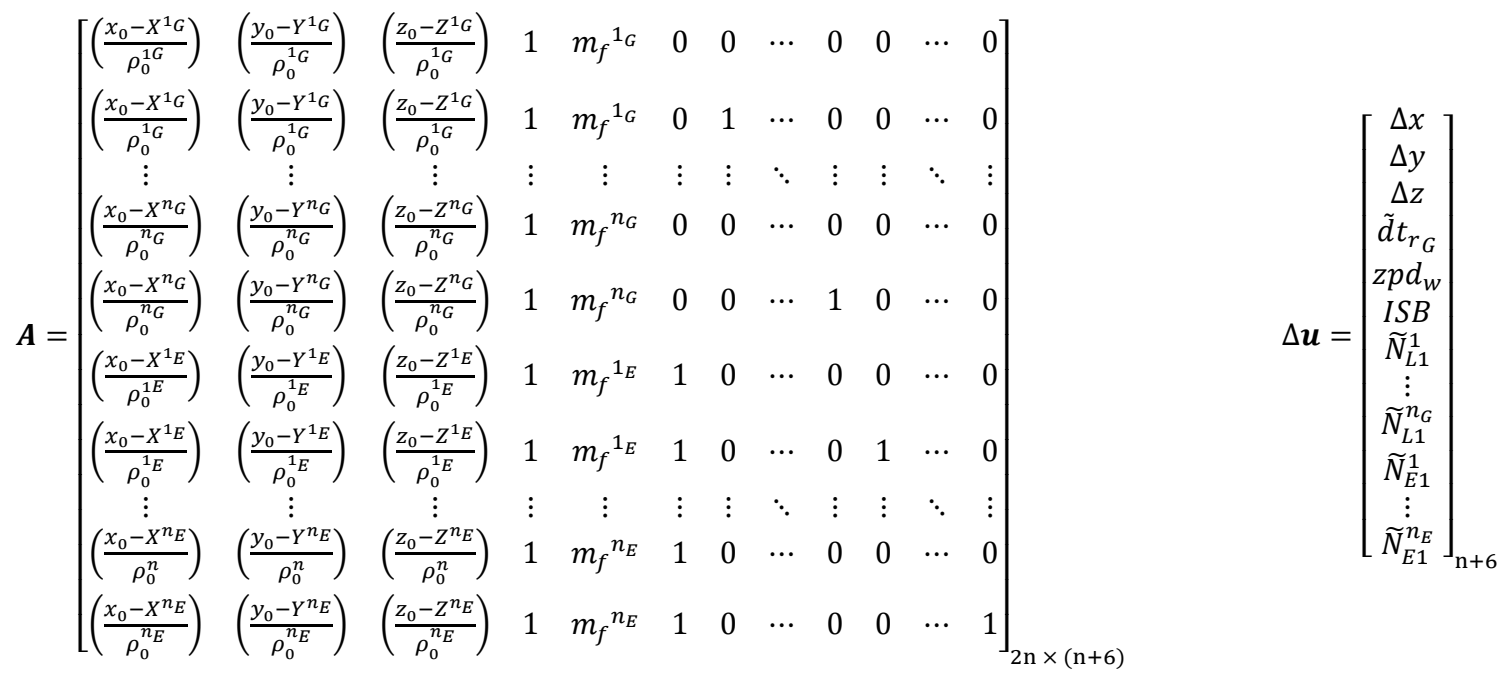

where $n_{G}$ refers to the number of visible GPS satellites; $n_{E}$ refers to the number of visible Galileo satellites; $n=n_{G}+n_{E}$ is the total number of the observed satellites for both GPS and Galileo systems; $x_{0}, y_{0}$ and $z 0$ are the approximate receiver coordinates; $X^{j_{G}}, Y^{j_{G}}, Z^{j_{G}}, j=1,2, \ldots, n_{G}$ are the known GPS satellite coordinates; $X^{k_{E}}, Y^{k_{E}}, Z^{k_{E}}, k=1,2, \ldots, n_{E}$ are the known Galileo satellite coordinates; $\rho_{0}$ is the approximate receiver-satellite range. The unknown parameters in the above system are the corrections to the receiver coordinates, $\Delta x, \Delta y$, and $\Delta z$, the biased receiver clock error $\tilde{d} t_{r_{G}}$, the wet component of the tropospheric zenith path delay $z p d_{w}$, the inter-system bias $I S B$, and the non-integer ambiguity parameters $\widetilde{N}$. It should be pointed out that the number of unknown parameters in the above system equals $n_{G}+n_{E}+6$, while the number of equations equals $2 n_{G}+2 n_{E}$. This means that the redundancy equals $n_{G}+n_{E}-6$. In other words, at least 6 mixed satellites are needed for the solution to exist. In comparison with the GPS-only undifferenced scenario, which requires a minimum of 5 satellites for the solution to exist, the 
addition of Galileo satellites increases the redundancy by $n_{E}-1$. In other words, we need a minimum of two Galileo satellites in order to contribute to the solution.

When a GPS satellite is selected as a reference to form the BSSD for both GPS and Galileo observations, the design matrix $\boldsymbol{A}$ and the vector of corrections $\boldsymbol{\Delta} \boldsymbol{u}$ take the form:

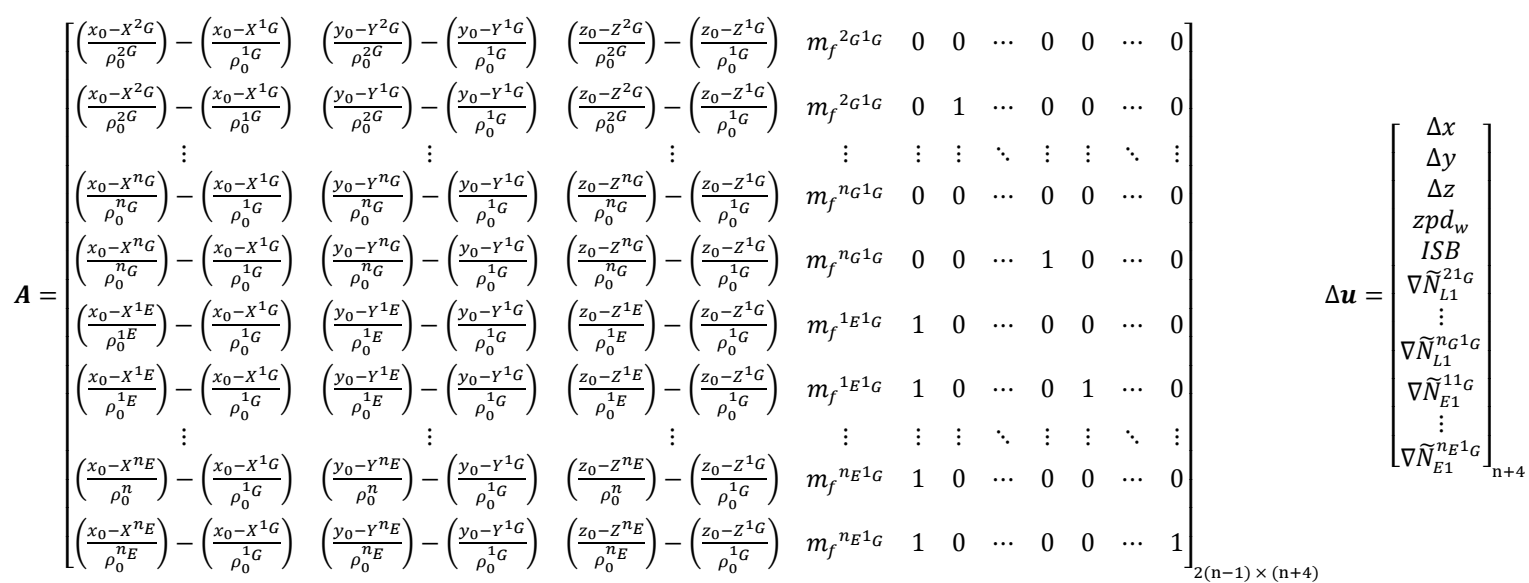

where $l_{G}$ refers to the GPS reference satellite. All other parameters are as defined above. The advantage of the above system (3.41) is that the number of unknown parameters is reduced by two (i.e., becomes $n_{G}+n_{E}+4$ ), in comparison with the un-differenced scenario. This, however, comes at the expense of reducing the number of BSSD observation equations to $2\left(n_{G}+n_{E}-1\right)$. As such, the redundancy remains unchanged and equals $n_{G}+n_{E}-6$. Similarly, we need a minimum of two Galileo satellites in order to contribute to the solution. By analogy, the use of a Galileo satellite as a reference to form the BSSD for both GPS and Galileo observations leads to:

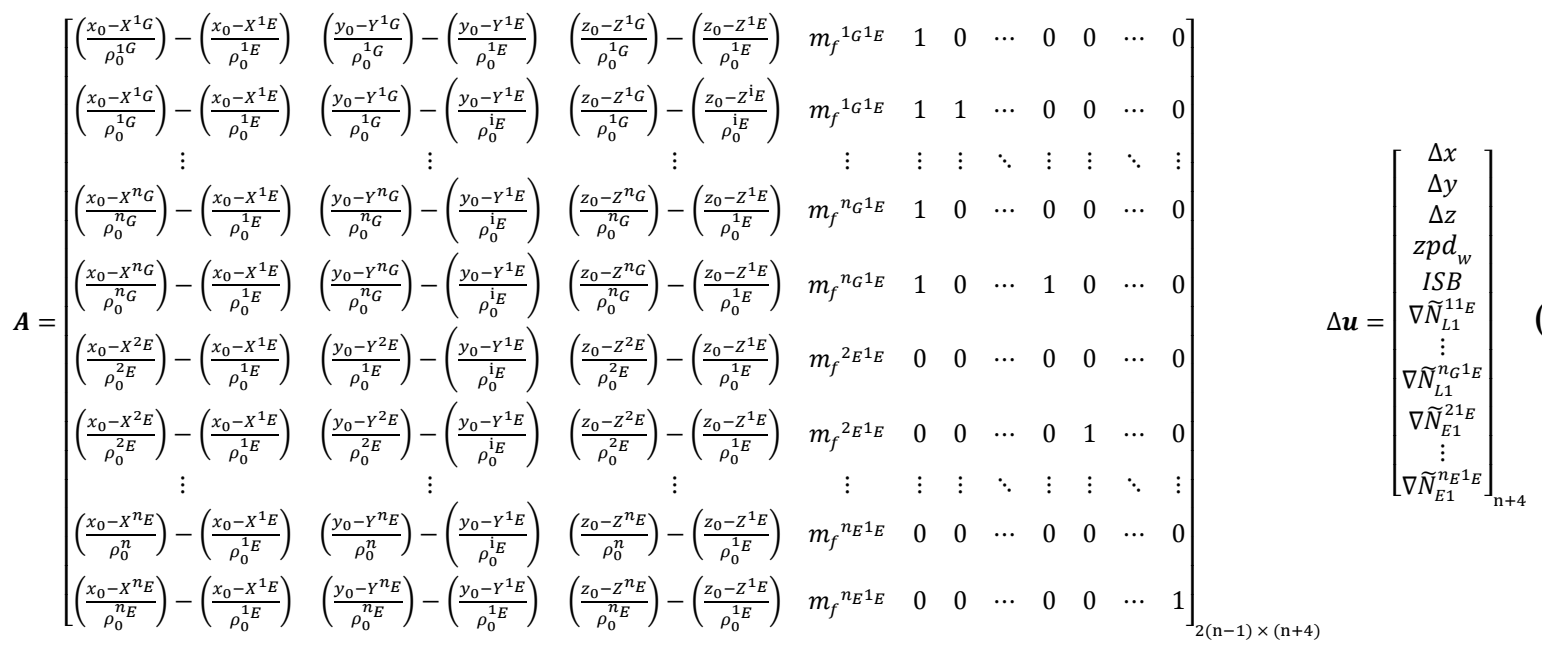


where $l_{E}$ refers to the Galileo reference satellite. All other parameters are as defined above. Similar to the above BSSD scenario, the redundancy remains unchanged and equals $n_{G}+n_{E}-6$.

When two reference satellites are selected to form the BSSD, i.e., per-constellation BSSD, the design matrix $\boldsymbol{A}$ and the vector of corrections $\boldsymbol{\Delta} \boldsymbol{u}$ take the form:

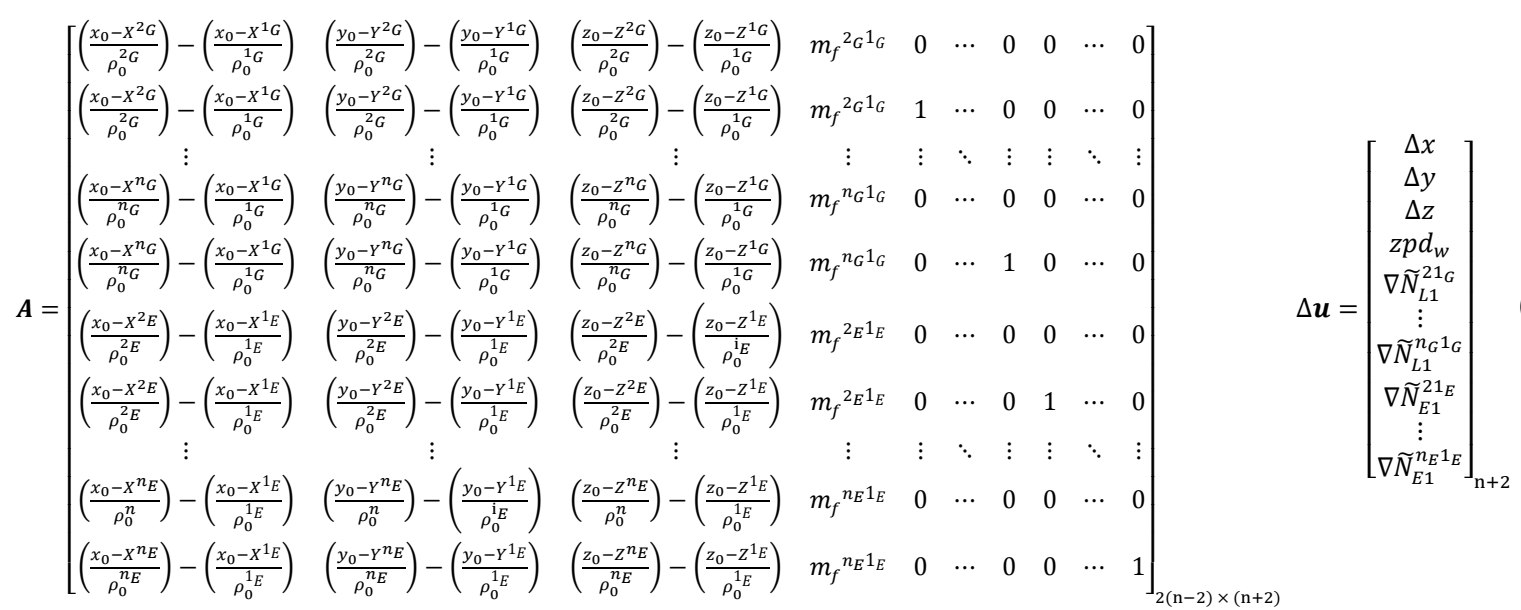

The major advantage of the above per-constellation (or loose combination) system is that the modified receiver clock error and the inter-system bias are cancelled out. In addition, the total number of unknown parameters is reduced by 4 to become $n_{G}+n_{E}+2$, in comparison with the undifferenced scenario. This, however, comes at the expense of reducing the number of BSSD observation equations to $2\left(n_{G}+n_{E}-2\right)$. This means that the redundancy remains unchanged and equals $n_{G}+n_{E}-6$. Similar to the previous scenarios, the redundancy for the GPS-only scenario is still $n_{G}-5$ and the addition of Galileo satellites increased the overall redundancy to $n_{E}-1$. As such, we still need a minimum of two Galileo satellites in order to contribute to the solution. However, as indicated by Paziewski and Wielgosz (2014), the adjustment model is stronger through the tight combination, i.e., when a single satellite is used as a reference.

\subsection{RESULTS AND DISCUSSION}

To verify the developed GPS/Galileo PPP model, GPS and Galileo measurements at six welldistributed stations (Figure 3.1) were selected from the IGS tracking network (Dow et al. 2009). Those stations are occupied by GNSS receivers, which are capable of simultaneously tracking 
the GPS and Galileo constellations. The analysis is performed on two different days January 1, 2014 and July 8, 2014 for all stations shown in Figure 3.1.

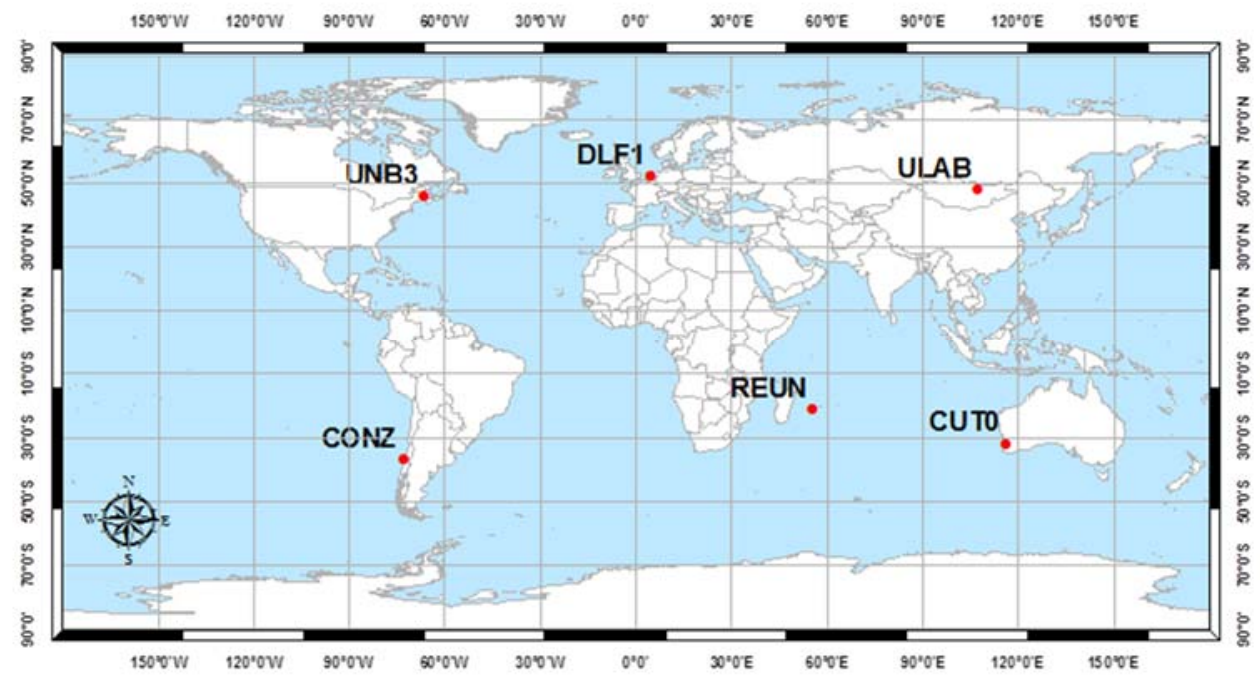

Figure 3.1 Analysis stations

The sampling interval for all data sets is 30 seconds, while the time span used in the analysis is three hours, which is selected at different times of the day to ensure that the four Galileo satellites are visible at each station. GPSPace PPP software of Natural Resources Canada (NRCan) was modified to enable a GPS/Galileo PPP solution as described above. The positioning results for stations CONZ, and CUT0 (January 1, 2014) and stations DLF1, and UNB3 (July 8, 2014) are presented below. Similar results are obtained from the other stations. However, a summary of the convergence times is presented below for all stations.

The single-frequency GPS/Galileo PPP solution is implemented through combining the GPS L1 signal with the Galileo E1 signal. As mentioned earlier, three different scenarios are considered when processing the data sets with the BSSD model, namely (1) a GPS satellite is selected as a reference satellite for both GPS and Galileo observables; (2) a Galileo satellite is selected as a reference satellite for both GPS and Galileo observables; and (3) two reference satellites are selected: a GPS reference satellite for the GPS observables and a Galileo satellite for the Galileo observables. To assess the PPP solution accuracy of the developed single frequency model, undifferenced dual-frequency ionosphere-free linear combination of GPS/Galileo PPP is used as a 
reference. Figure 3.2 shows the reference solution for July 8, 2014, as an example. As can be seen, the dual-frequency GPS/Galileo PPP solution has a sub-decimeter positioning accuracy with approximately 15 minutes convergence time (i.e., the time that the solution takes to converge to a decimetre-level positioning accuracy).
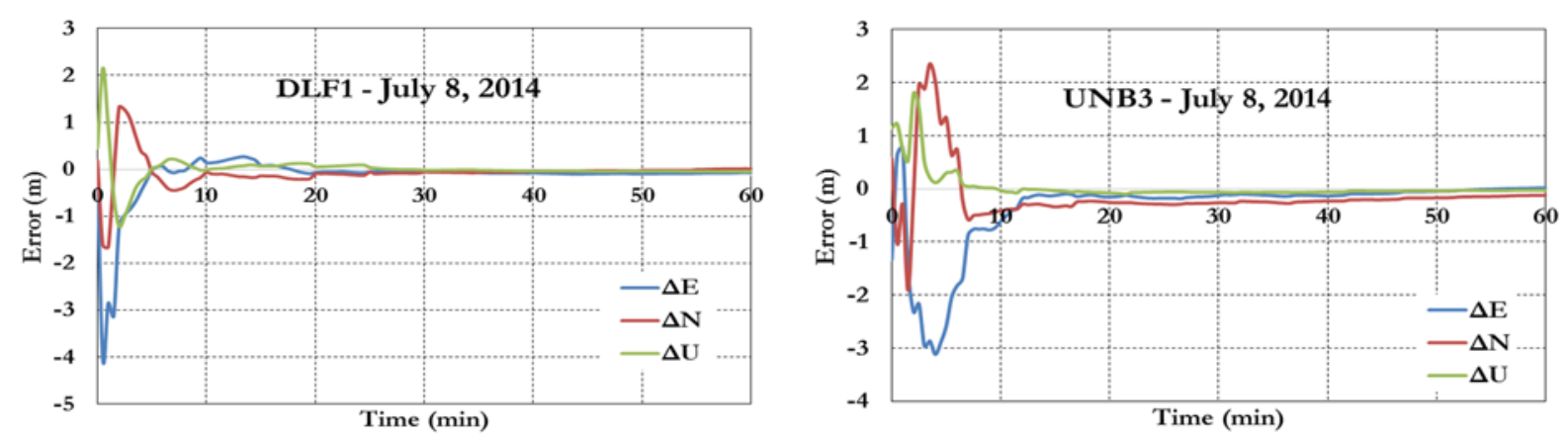

Figure 3.2 Un-differenced ionosphere-free GPS/Galileo PPP solution

Figure 3.3 shows the PPP results for the un-differenced single frequency GPS/Galileo model. As can be seen, the single frequency GPS/Galileo PPP solution shows a decimetre level accuracy and approximately 100 minutes convergence time. It should be emphasized that the exponential function is used to model the stochastic part of the mathematical model. As shown in Afifi and El-Rabbany (2013), the use of the exponential function improves the precision of the PPP solution by about $30 \%$, in comparison with the sine function.
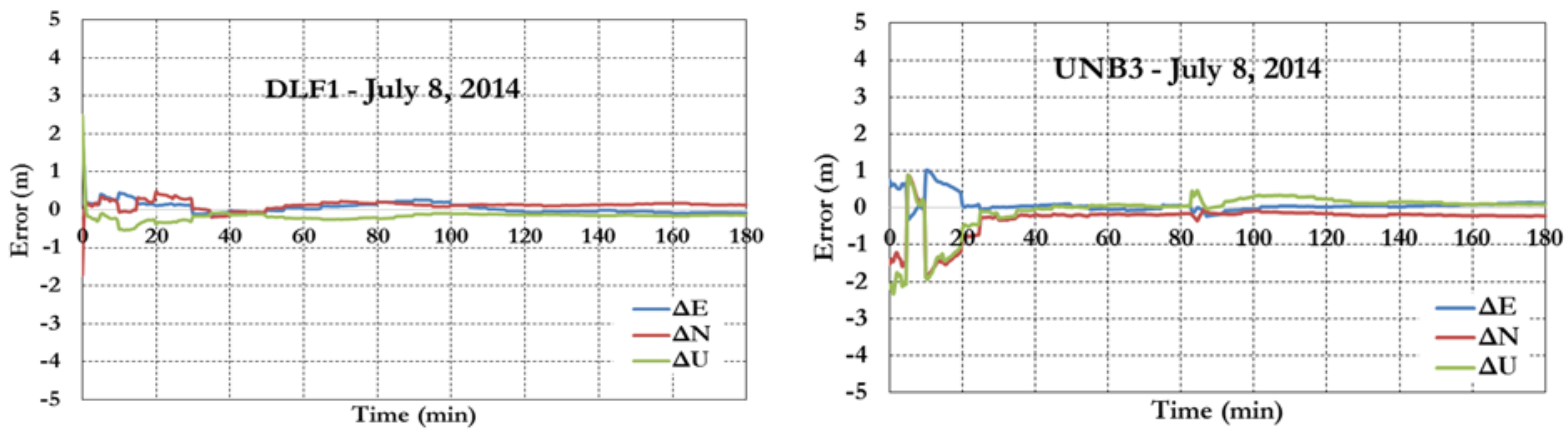

Figure 3.3 Un-differenced single frequency GPS/Galileo PPP solution

Figure 3.4 shows the results of the single-frequency BSSD GPS/Galileo PPP model, when a GPS satellite is selected as a reference for both GPS and Galileo observables. As can be seen, similar 
to the un-differenced counterpart, a sub-decimeter level positioning accuracy is obtained. However, a reduction of about $35 \%$ in the convergence time is obtained through the BSSD model, in comparison with the un-differenced single frequency GPS/Galileo solution.
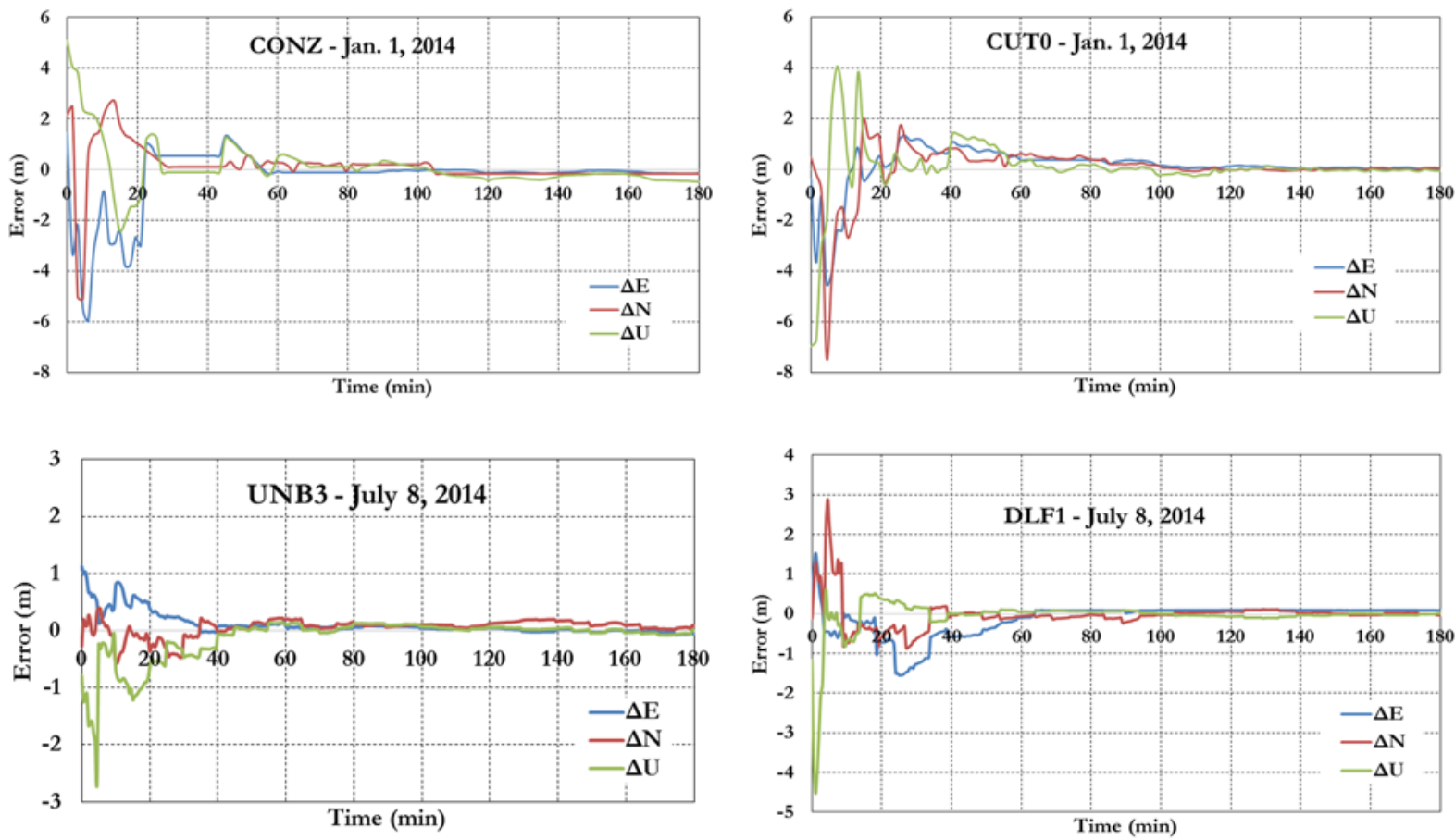

Figure 3.4 BSSD GPS/Galileo PPP solution using a GPS reference satellite

Figure 3.5 shows the results of the BSSD GPS/Galileo PPP solution when a Galileo satellite is selected as a reference for both GPS and Galileo observables. As expected, similar results to the previous scenario are obtained. This similarity is due mainly to the fact that both BSSD models use tight combination with similar relative satellite geometry and same redundancy number.
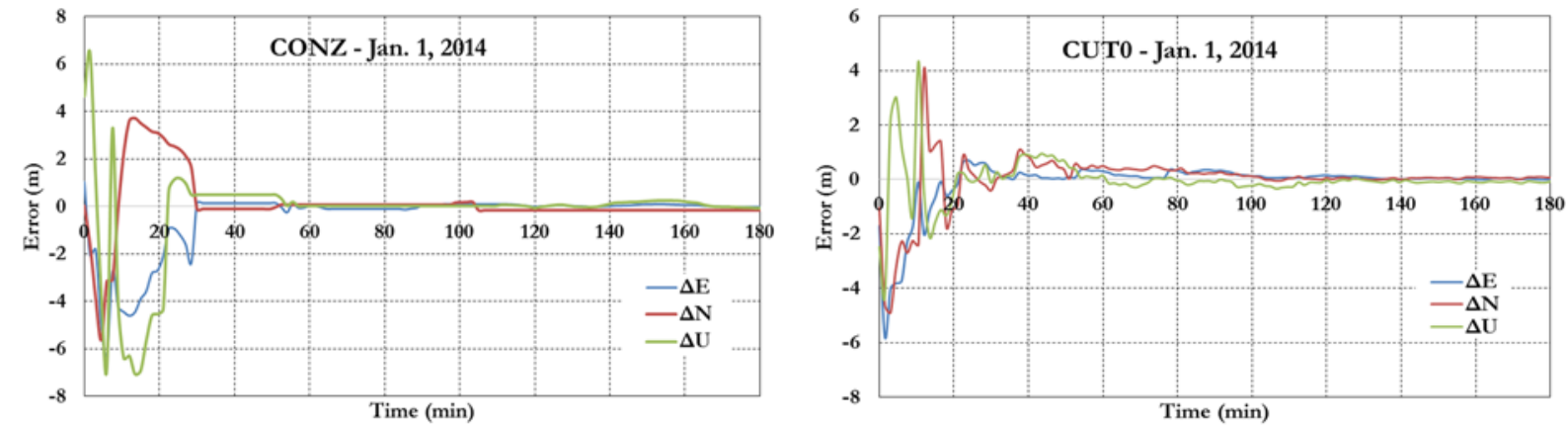

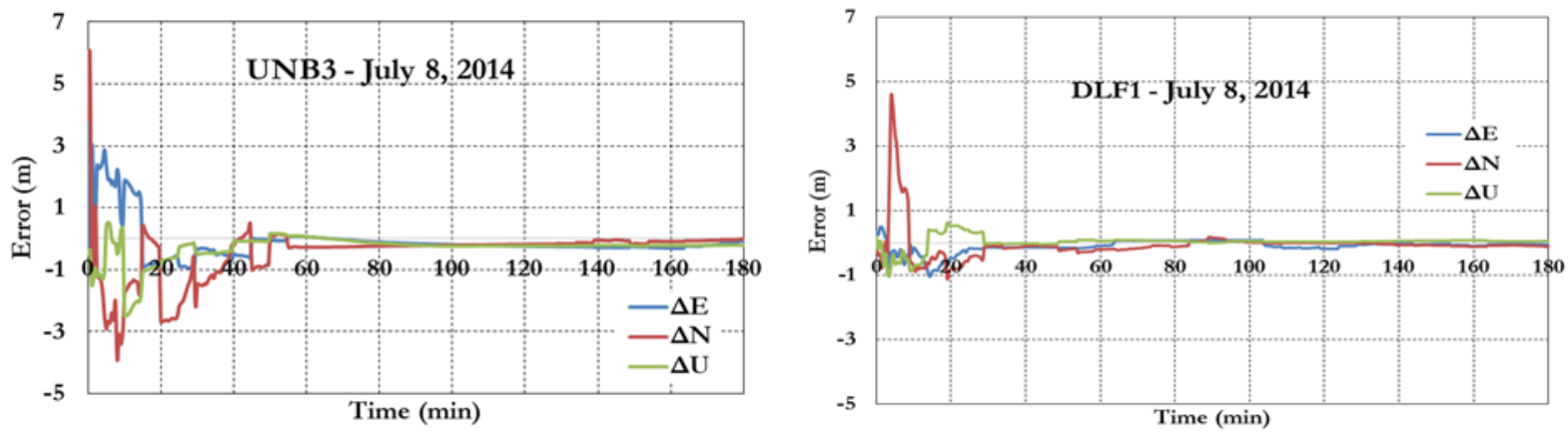

Figure 3.5 BSSD GPS/Galileo PPP solution using a Galileo reference satellite

The results of the third BSSD model scenario, i.e., when a GPS satellite and a Galileo satellite are selected as references for the GPS and Galileo observables, respectively, are shown in Figure 3.6. As can be seen, similar to the previous two scenarios, a sub-decimeter level positioning accuracy is obtained. However, only a $15 \%$ reduction in the convergence time is obtained, in comparison with the un-differenced model solution. This moderate improvement in the convergence time is likely attributed to the fact that the loose combination is weaker than the tight combination, as indicated above. In addition, as the ionospheric delay would not be modelled sufficiently through the GIM, a residual component remains, which would be mapped differently into the unknown parameters of the BSSD models.
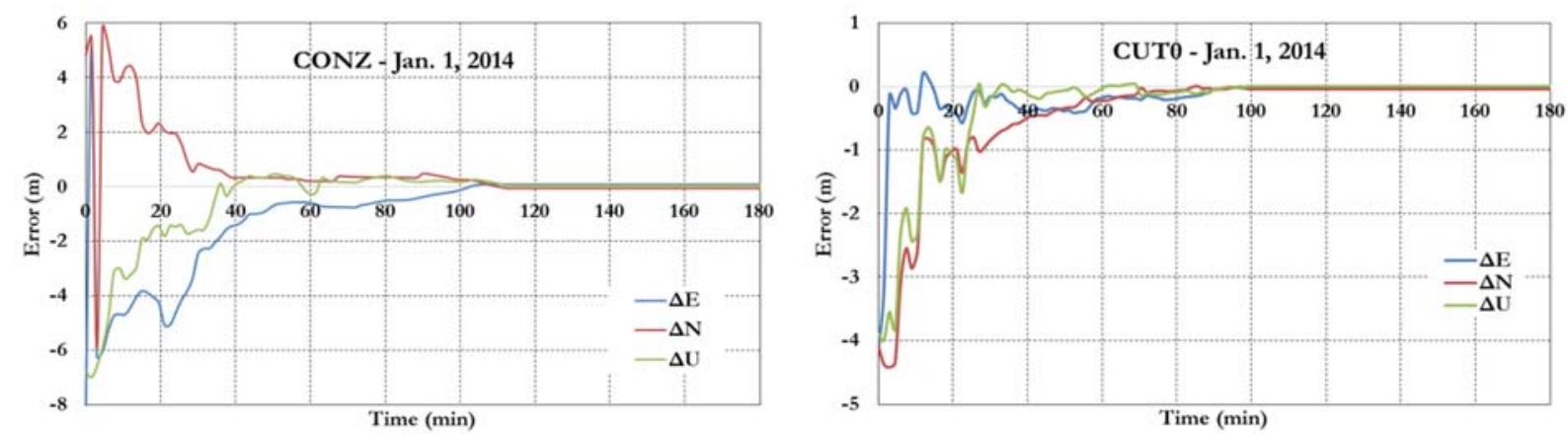

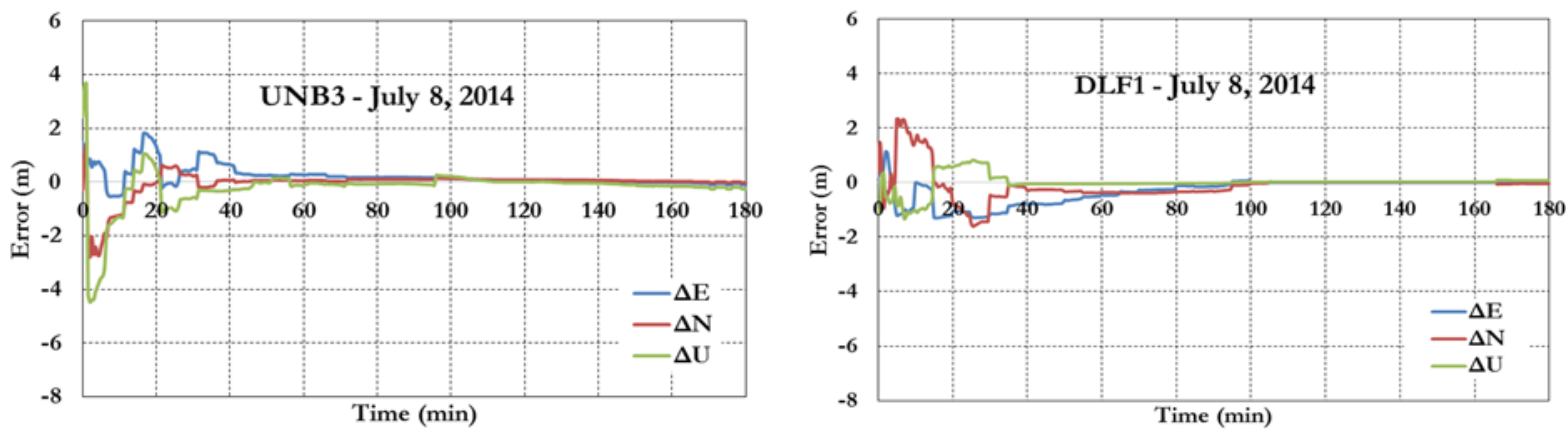

Figure 3.6 BSSD GPS/Galileo PPP solution using two reference satellites

Figure 3.7 shows a summary of the standard deviations (STD) for the obtained station coordinates, which are extracted from the solution every 20 minutes. The results of the STD show that the un-differenced GPS/Galileo solution takes approximately 100 minutes to reach the decimetre level accuracy. However, this is reduced to about 65 minutes when BSSD with one reference satellite is used. On the other hand, when BSSD with two reference satellites is used, the solution takes 85 minutes to reach the decimetre level accuracy. As discussed above, this is likely attributed to the relatively weaker adjustment model of the loose combination.

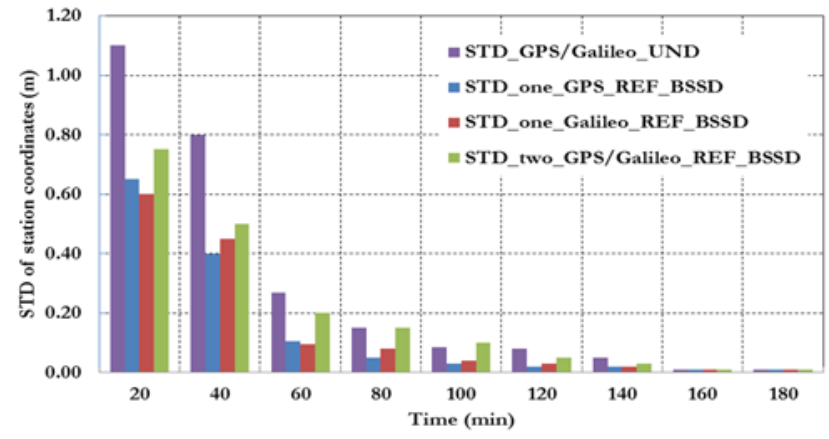

Figure 3.7 Summary of standard deviations of obtained station coordinates

Figure 3.8 summarizes the convergence times of all 24 cases considered in our analysis. As shown in Figure 3.9, as far as the solution convergence time is concerned, the best results are obtained when the BSSD model with one reference satellite (i.e., a tight combination) is used. 


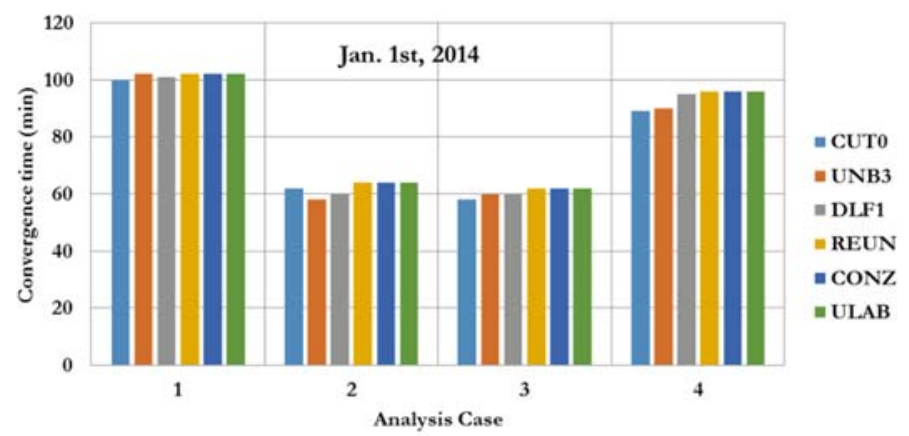

Figure 3.8 Summary of convergence times for all data sets analysed. (1) un-differenced model; (2) BSSD model with a GPS satellite as a reference; (3) BSSD model with a Galileo satellite as a reference; (4) BSSD model with both a GPS and a Galileo as reference satellite

The inter-system bias for the various receivers is obtained as a by-product of the PPP solution of the un-differenced and tight combination scenarios. Figure 3.9 summarises the results of the ISB for all stations, based on the BSSD single-frequency GPS/Galileo PPP model. The results are almost identical to those obtained the through the un-differenced single-frequency GPS/Galileo PPP model.

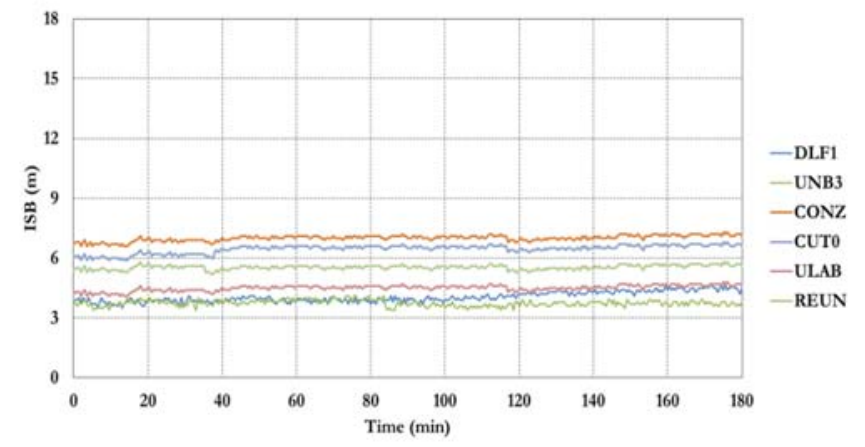

Figure 3.9 Summary of ISB results

As shown in Figure 3.9, the values of the ISB are stable over the observation time spans. Differences of up to $3 \mathrm{~m}$ can be observed, which indicate that the ISB is receiver/firmware dependent. 


\subsection{CONCLUSIONS}

A new PPP model, which combines single frequency GPS and Galileo system observations in BSSD mode, has been introduced in this paper. Three scenarios have been considered when forming BSSD, namely a GPS satellite is selected as a reference, a Galileo satellite is selected as a reference, and two satellites, one GPS and one Galileo, are selected as references. It has been shown that a sub-decimetre level positioning accuracy can be obtained with both of the undifferenced and BSSD single-frequency GPS/Galileo PPP models. However, the PPP solution of the un-differenced model takes about 100 minutes to converge to a decimetre level positioning accuracy. The convergence time of the single-frequency GPS/Galileo PPP solution is improved by $35 \%$ and $15 \%$ when BSSD with tight and loose combinations are used, respectively. The moderate improvement in the solution convergence time obtained with the loose combination is likely attributed to its relatively weaker adjustment model in comparison with the tight combination.

The values of the ISB have been obtained for various days and receiver types. Almost identical results have been obtained with both of the un-differenced and BSSD (tight combination) modes. It has been found that the values of the ISB are largely stable over the observation time spans. However, differences of up to $3 \mathrm{~m}$ have been observed, which suggest that the ISB is receiver/firmware dependent. 


\section{CHAPTER 4}

\section{AN IMPROVED BETWEEN-SATELLITE SINGLE-DIFFERENCE PRECISE POINT POSITIONING MODEL FOR COMBINED GPS/GALILEO OBSERVATIONS}

This chapter describes a dual-frequency GPS/Galileo PPP model, which uses un-differenced and BSSD (with both loose and tight combinations) models. It is originally published as:

Afifi, A., El-Rabbany, A. 2015. An improved between-satellite single-difference precise point positioning model for combined GPS/Galileo observations. Journal of Applied Geodesy, 9(2), 101-111, doi: 10.1515/jag-2014-0030.

Modifications to the original manuscript were made only for proper identification of sections, figures and tables, as well as to assure the uniformity of symbol and equation notation throughout this dissertation. 


\begin{abstract}
This paper introduces a new model for precise point positioning (PPP), which combines dualfrequency GPS and Galileo observations. Our model is based on the between-satellite singledifference (BSSD) linear combination, which cancels out some receiver-related biases, including receiver clock error and non-zero initial phase bias of the receiver's oscillator. Two different scenarios are considered when forming BSSD linear combinations. In the first scenario, either a GPS or a Galileo satellite is selected as a reference for both GPS and Galileo observables. The second scenario, on the other hand, selects two reference satellites: a GPS reference satellite for the GPS observables and a Galileo satellite for the Galileo observables. Natural Resources Canada's GPSPace PPP software is modified to enable a combined GPS/Galileo PPP solution and to handle the newly introduced biases. A total of 12 data sets representing two-day GPS/Galileo measurements at six IGS stations are processed to verify the developed PPP model. Precise satellite orbit and clock products from the IGS-MGEX network are used to correct both of the GPS and Galileo measurements. It is shown that using one reference satellite to form the BSSD linear combinations improves the precision of the estimated parameters by about $25 \%$ compared with the GPS-only PPP solution. When two reference satellites are used, however, the precision of the estimated parameters improves by about 50\% compared with the GPS-only PPP solution. Additionally, the solution convergence time is reduced to 10 minutes for both BSSD scenarios, which represents about 50\% improvement in comparison with the GPS-only PPP solution.
\end{abstract}

\title{
4.1. INTRODUCTION
}

Precise point positioning (PPP) technique proved to be capable of providing positioning solutions at the sub-decimeter- and decimeter-level accuracy in static and kinematic modes, respectively. PPP accuracy and convergence time are controlled by the ability to mitigate all potential errors and biases. A number of un-differenced and between-satellite single-difference (BSSD) GPS PPP models have been developed since early 2000 (see for example, Kouba and Héroux, 2001; Colombo et al., 2004; Ge at el., 2008; Collins at al., 2010). For a single global navigation satellite system (GNSS) constellation, BSSD linear combination cancels out all receiver-related biases, assuming that multipath does not exist. Elsobeiey and El-Rabbany (2014) 
showed that, as a result of eliminating the receiver-related biases, a faster PPP convergence time is obtained through the BSSD GPS PPP model.

More recently, Afifi and El-Rabbany (2015) showed that combining the un-differenced GPS and Galileo observations in a PPP model improves the solution convergence time by about $25 \%$, in comparison with the GPS-only counterpart. In addition, they showed that the inter-system bias is largely constant over a one-hour observation time span, which they used in their analysis, with a magnitude ranging from 30 to 60 nanoseconds depending on the GNSS receiver type. Building on earlier research related to the GPS PPP ambiguity resolution, Tegedor et al. (2014) estimated the uncalibrated hardware delays (UHDs) for Galileo satellites through the adjustment of a network of GNSS reference stations, for the purpose of fixing the Galileo PPP ambiguity parameters. Using a common set of precise orbits, clock corrections and UHDs, they processed data from a number of stations occupied by different receiver types, both with fixed and float ambiguity parameters. However, the improvement in the PPP positioning accuracy was marginal, which is mainly attributed to the limited number of Galileo satellites presently in the constellation.

This paper develops a PPP model, which combines GPS and Galileo dual-frequency observables using BSSD mode. We consider two scenarios when forming the BSSD linear combination, namely a tight and a loose combination. In the first scenario, either a GPS or a Galileo satellite is selected as a reference for both GPS and Galileo observables. The second scenario, which is also referred to as per constellation combination, considers two reference satellites: a GPS reference satellite for the GPS observables and a Galileo satellite for the Galileo observables (Odijk and Teunissen, 2013). Precise orbits and clock corrections from the International GNSS Service multi-GNSS experiment (IGS-MEGX) network are used to account for GPS and Galileo satellite orbit and clock errors (Montenbruck et al., 2014). As these products are presently referenced to the GPS time and since we use mixed GNSS receivers that also use GPS time as a reference, the GGTO is cancelled out in our model. The inter-system bias is either cancelled out through differencing the observations or is treated as an additional unknown parameter. The hydrostatic component of the tropospheric zenith path delay is modelled through the Hopfield model, while the wet component is considered as an additional unknown parameter (Hopfield, 1972; 
Hofmann-Wellenhof et al., 2008). All remaining errors and biases are accounted for using existing models as shown in Kouba (2009). It is shown that the newly developed GPS/Galileo PPP model improves the precision of the estimated parameters by about $50 \%$ and $25 \%$, in comparison with the un-differenced GPS-only model, when the loose and the tight combinations are used, respectively. In addition, the solution convergence time is reduced to 10 minutes for both BSSD scenarios, which represents about 50\% improvement in comparison with the GPSonly PPP solution.

\subsection{GPS/GALILEO PPP MATHEMATICAL MODEL}

Following Hofmann-Wellenhof et al., (2008) and Afifi and El-Rabbany (2015), the basic mathematical model underlying dual-frequency PPP is defined by the ionosphere-free linear combinations as shown in Equations 4.1-4.5.

$$
\begin{aligned}
& P_{G_{I F}}=\rho_{G}+c\left[d t_{r G}-d t^{s}\right]+c\left[\alpha_{G} d_{P 1}-\beta_{G} d_{P 2}\right]_{r}+c\left[\alpha_{G} d_{P 1}-\beta_{G} d_{P 2}\right]^{s}+T_{G}+\varepsilon_{P G_{I F}} \\
& P_{G_{I F}(C l / P 2)}=\rho_{G}+c\left[d t_{r G}-d t^{s}\right]+c\left[\alpha_{G} d_{C l}-\beta_{G} d_{P 2}\right]_{r}+c\left[\alpha_{G} d_{C l}-\beta_{G} d_{P 2}\right]^{s}+T_{G}+\varepsilon_{(C 1 / P 2) G_{I F}} \\
& P_{E_{I F}}=\rho_{E}+c\left[d t_{r G}-G G T O-d t^{s}\right]+c\left[\alpha_{E} d_{E l}-\beta_{E} d_{E 5 a}\right]_{r}+c\left[\alpha_{E} d_{E l}-\beta_{E} d_{E S a}\right]^{s}+T_{E}+\varepsilon_{E_{I F}} \\
& \Phi_{G_{I F}}=\rho_{G}+c\left[d t_{r G}-d t^{s}\right]+c\left[\alpha_{G} \delta_{L 1}-\beta_{G} \delta_{L 2}\right]_{r}+c\left[\alpha_{G} \delta_{L 1}-\beta_{G} \delta_{L 2}\right]^{s}+T_{G}+N_{G_{I F}}+\phi_{r 0_{G F}}+\phi_{0_{G F}}^{s}+\varepsilon_{\Phi G_{I F}} \\
& \Phi_{E_{I F}}=\rho_{E}+c\left[d t_{r G}-G G T O-d t^{s}\right]+c\left[\alpha_{E} \delta_{E I}-\beta_{E} \delta_{E 5 a}\right]_{r}+c\left[\alpha_{E} \delta_{E I}-\beta_{E} \delta_{E 5 a}\right]^{s}+T_{E}+N_{E_{E F}}+\phi_{r_{0} 0_{F F}}+\phi_{E_{E F}}^{5}+\varepsilon_{\Phi E_{I F}}
\end{aligned}
$$

where the subscripts $G$ and $E$ refer to the GPS and Galileo satellite systems, respectively; $P_{G_{I F}}$ and $P_{E_{I F}}$ are the ionosphere-free pseudoranges in meters for GPS and Galileo systems, respectively; $\Phi_{G_{I F}}$ and $\Phi_{E_{I F}}$ are the ionosphere-free carrier phase measurements in meters for GPS and Galileo systems, respectively; $\rho$ is the true geometric range from receiver at reception time to satellite at transmission time in meters; $d t_{r}, d t^{s}$ are the clock errors in seconds for the receiver at signal reception time and the satellite at signal transmission time, respectively; $d_{P 1 r}, d_{P 2 r}, d_{C 1 r}$, $d_{E 1 r}, d_{E 5 a r}$ are frequency-dependent code hardware delays for the receiver at reception time in seconds; $d_{P 1}{ }^{s}, d_{P 2}{ }^{s}, d_{C 1}{ }^{s}, d_{E 1}{ }^{s}, d_{E S a}{ }^{s}$ are frequency-dependent code hardware delays for the satellite at transmission time in seconds; $\delta_{L 1}, \delta_{L 2}, \delta_{E 1}, \delta_{E 5 a}$ are frequency-dependent carrier- 
phase hardware delays for the receiver at reception time in seconds; $\delta_{L 1}{ }^{s}, \delta_{L 2}{ }^{s}, \delta_{E 1}{ }^{s}, \delta_{E S a}{ }^{s}$ are frequency-dependent carrier-phase hardware delays for the satellite at transmission time in seconds; $T$ is the tropospheric delay in meter; $N_{G_{I F}}, N_{E_{I F}}$ are the ionosphere-free linear combinations of the ambiguity parameters for both GPS and Galileo carrier-phase measurements in meters, respectively (Equations 6 and 7); $\phi_{r 0_{G_{I F}}}, \phi_{0_{G_{I F}}^{S}}^{S}, \phi_{r 0_{E_{I F}}}, \phi_{0_{E_{I F}}^{S}}$ are ionosphere-free linear combinations of frequency-dependent initial fractional phase biases in the receiver and satellite channels for both GPS and Galileo in meters, respectively; $c$ is the speed of light in vacuum in meter per second; $\varepsilon_{P_{I F}}, \varepsilon_{(C 1 / P 2) G_{I F}}, \varepsilon_{E_{I F}}, \varepsilon_{\Phi G_{I F}}, \varepsilon_{\Phi E_{I F}}$ are the ionosphere-free linear combinations of the relevant noise and un-modeled errors in meter; $\alpha_{G}, \beta_{G}, \alpha_{E}, \beta_{E}$ are the ionosphere-free linear combination coefficients for both GPS and Galileo, which are given, respectively, by: $\alpha_{G}=\frac{f_{1}^{2}}{f_{1}^{2}-f_{2}^{2}}, \beta_{G}=\frac{f_{2}^{2}}{f_{1}^{2}-f_{2}^{2}}, \alpha_{E}=\frac{f_{E 1}^{2}}{f_{E 1}^{2}-f_{E S a}^{2}}, \beta_{E}=\frac{f_{E S a}^{2}}{f_{E 1}^{2}-f_{E S a}^{2}}$.

where $f_{1}$ and $f_{2}$ are the GPS $\mathrm{L}_{1}$ and $\mathrm{L}_{2}$ signal frequencies; $f_{E 1}$ and $f_{E 5 a}$ are the Galileo $\mathrm{E}_{1}$ and E5a signal frequencies. The ionosphere-free linear combinations of the ambiguity parameters for both GPS and Galileo carrier-phase measurements are defined by:

$$
\begin{aligned}
& N_{G_{I F}}=\lambda_{G_{I F}}\left[f_{1} N_{1}-f_{2} N_{2}\right] \\
& N_{E_{I F}}=\lambda_{E_{I F}}\left[f_{E 1} N_{E 1}-f_{E 5 a} N_{E 5 a}\right]
\end{aligned}
$$

where $\lambda_{G I F}$ and $\lambda_{E I F}$ are the ionosphere-free linear combinations of the wavelengths in meters, which are given, respectively, by $\lambda_{G_{I F}}=\frac{c}{f_{1}^{2}-f_{2}^{2}}$ and $\lambda_{E_{I F}}=\frac{c}{f_{E 1}^{2}-f_{E S a}^{2}} ; N_{1}, N_{2}$ are the integer ambiguity parameters of GPS signals L1 and L2, respectively; $N_{E 1}, N_{E 5 a}$ are the integer ambiguity parameters of Galileo signals E1 and E5a, respectively.

As indicated earlier, precise orbit and satellite clock corrections from the IGS-MGEX network are used to correct both of the GPS and Galileo measurements. It should be pointed out that such products are presently referenced to the GPS time frame (Montenbruck et al., 2014). As well, the IGS-MGEX precise GPS satellite clock corrections include the effect of the ionosphere-free linear combination of the satellite hardware delays of L1/L2 P(Y) code, while the Galileo counterpart include the effect of the ionosphere-free linear combination of the satellite hardware 
delays of the Galileo E1/E5a pilot code (Montenbruck et al., 2014). Applying the precise clock corrections to Equations 4.1 to 4.5, we obtain:

$$
\begin{aligned}
& P_{G_{I F}}=\rho_{G}+c\left[d t_{r G}-d t_{p r e c}^{s}\right]+c\left[\alpha d_{P 1}-\beta d_{P 2}\right]_{r}+T_{G}+\varepsilon_{P G_{I F}} \\
& P_{G_{I F}(C l / P 2)}=\rho_{G}+c\left[d t_{r G}-d t_{p r e c}^{s}\right]+c\left[\alpha d_{C l}-\beta d_{P 2}\right]_{r}+\alpha D C B_{C 1 / P 1}^{S}+T_{G}+\varepsilon_{P G_{I F}} \\
& P_{E_{I F}}=\rho_{E}+c\left[d t_{r G}-d t_{p r e c}^{s}\right]+c\left[\alpha d_{E I}-\beta d_{E S a}\right]_{r}+T_{E}+\varepsilon_{E_{I F}} \\
& \Phi_{G_{F F}}=\rho_{G}+c d t_{r G}-c\left[d t_{p r e c}^{s}+\left[\alpha d_{P I}-\beta d_{P 2}\right]^{s}\right]+c\left[\alpha \delta_{L l}-\beta \delta_{L 2}\right]_{r}+c\left[\alpha \delta_{L l}-\beta \delta_{L 2}\right]^{s}+T_{G}+N_{G_{I F}}+\phi_{r 0_{G F}}+\phi_{\sigma_{G F}}^{s}+\varepsilon_{\Phi G_{I F}} \\
& \Phi_{E_{I F}}=\rho_{E}+c d t_{r G}-c\left[d t_{p r e c}^{s}+\left[\alpha d_{E I}-\beta d_{E S a}\right]^{s}\right]+c\left[\alpha \delta_{E I}-\beta \delta_{E S a}\right]_{r}+c\left[\alpha \delta_{E I}-\beta \delta_{E S a}\right]^{s}+T_{E}+N_{E_{I F}}+\phi_{r E_{E F}}+\phi_{\delta_{E F}}^{s}+\varepsilon_{\Phi E_{I F}}
\end{aligned}
$$

where $D C B^{s} C I / P 1$ is the GPS C1/P1 differential code bias. To simplify Equations 4.8 to 4.12 , the receiver and satellite hardware delays are written as:

$$
\begin{aligned}
& b_{r_{P}}=c\left[\alpha d_{P 1}-\beta d_{P 2}\right]_{r} \quad b_{P}^{s}=c\left[\alpha d_{P 1}-\beta d_{P 2}\right]^{s} \\
& b_{r_{C}}=c\left[\alpha d_{C 1}-\beta d_{P 2}\right]_{r} \quad b_{C}^{s}=c\left[\alpha d_{C 1}-\beta d_{P 2}\right]^{s} \\
& b_{r_{E}}=c\left[\alpha d_{E 1}-\beta d_{E 5 a}\right]_{r} \quad b_{E}^{s}=c\left[\alpha d_{E 1}-\beta d_{E 5 a}\right]^{s} \\
& b_{r_{Q \Phi}}=c\left[\alpha \delta_{L 1}-\beta \delta_{L 2}\right]_{r}+\phi_{r_{0} 0_{G F}} \quad b_{G \Phi}^{s}=c\left[\alpha \delta_{L 1}-\beta \delta_{L 2}\right]^{s}+\phi_{0_{G F}}^{s} \\
& b_{r_{E \Phi}}=c\left[\alpha \delta_{E 1}-\beta \delta_{E 5 a}\right]_{r}+\phi_{r 0_{E_{F F}}} \quad b_{E \Phi}^{s}=c\left[\alpha \delta_{E 1}-\beta \delta_{E 5 a}\right]^{s}+\phi_{0_{E_{F F}}}^{s}
\end{aligned}
$$

To avoid a rank-defect system, the GPS receiver clock error is conventionally lumped with the GPS ionosphere-free linear combination of the receiver differential code biases. As well, to maintain consistency in the estimation of a common receiver clock offset, we follow this convention when combining the ionosphere-free linear combination of GPS L1/L2 and Galileo E1/E5a observations. This, however, introduces an additional bias in the Galileo ionosphere-free PPP mathematical model, which represents the difference in the ionosphere-free linear combination of the receiver differential code biases of both systems. Such an additional bias is commonly known as the inter-system bias, which is referred to as $I S B$ in this paper. With the above consideration, the GPS and Galileo ionosphere-free linear combinations of both pseudorange and carrier-phase measurements can be written in a compact form as: 


$$
\begin{aligned}
& P_{G_{I F}}=\rho_{G}+\tilde{d}_{r G}-d t_{\text {prec }}^{s}+T_{G}+\varepsilon_{P G_{I F}} \\
& P_{G_{I F}(C / / P 2)}=\rho_{G}+\tilde{d} t_{r G}-d t_{p r e c}^{s}+\alpha D C B_{C l / P 1}^{s}+T_{G}+\varepsilon_{P G_{I F}} \\
& P_{E_{I F}}=\rho_{E}+\tilde{d} t_{r G}-d t_{p r e c}^{s}+I S B+T_{E}+\varepsilon_{E_{I F}} \\
& \Phi_{G_{I F}}=\rho_{G}+\tilde{d} t_{r G}-d t_{p r e c}^{s}+T_{G}+\tilde{N}_{G_{I F}}+\varepsilon_{\Phi G_{I F}} \\
& \Phi_{E_{I F}}=\rho_{E}+\tilde{d} t_{r G}-d t_{p r e c}^{s}+T_{E}+\tilde{N}_{E_{I F}}+I S B+\varepsilon_{\Phi E_{I F}}
\end{aligned}
$$

where $\tilde{d} t_{r G}$ represents the sum of the receiver clock error and hardware delay, i.e., $\tilde{d} t_{r G}=c d t_{r G}+b_{r_{P}} ; I S B$ is the inter-system bias, $I S B=b_{r_{E}}-b_{r_{P}} ; \tilde{N}_{G_{I F}}$ and $\tilde{N}_{E_{I F}}$ represent the ambiguity parameters lumped to the receiver and satellite hardware delays, i.e.,

$$
\begin{aligned}
& \tilde{N}_{G_{I F}}=N_{G_{I F}}+b_{r_{G \Phi}}-b_{r_{P}}+b_{G \Phi}^{s}-b_{P}^{s} \\
& \tilde{N}_{E_{I F}}=N_{E_{I F}}+b_{r_{E \Phi}}-b_{r_{P}}+b_{E \Phi}^{s}-b_{E}^{s}
\end{aligned}
$$

In our PPP mode, the Hopfield tropospheric correction model along with the Vienna mapping function are used to account for the hydrostatic component of the tropospheric delay (Hopfield, 1972; Boehm and Schuh, 2004). Other corrections are also applied, including the effect of ocean loading (Bos and Scherneck, 2014; IERS, 2010), Earth tide (Kouba, 2009), carrier-phase windup (Leick, 2004; Wu et al., 1993), Sagnac (Kaplan and Heagarty, 2006), relativity (HofmannWellenhof et al., 2008), and satellite and receiver antenna phase-center variations (Dow et al. 2009). The noise terms are modeled stochastically using an exponential model, as described in Afifi and El-Rabbany (2013). Applying the above corrections and accounting for the differential code biases, we can re-write Equations 4.13 to 4.17 as:

$$
\begin{aligned}
& \rho_{G}+\tilde{d}_{r G}+m_{f} \times z p d_{w}+\varepsilon_{P G_{I F}}-\tilde{P}_{G_{I F}}=0 \\
& \rho_{G}+\tilde{d}_{r G}+m_{f} \times z p d_{w}+\varepsilon_{P G_{I F}}-\tilde{P}_{G_{I F}(C l / P I)}=0 \\
& \rho_{E}+\tilde{d} t_{r G}+m_{f} \times z p d_{w}+I S B+\varepsilon_{E_{I F}}-\tilde{P}_{E_{I F}}=0 \\
& \rho_{G}+\tilde{d} t_{r G}+m_{f} \times z p d_{w}+\tilde{N}_{G_{I F}}+\varepsilon_{\Phi G_{I F}}-\tilde{\Phi}_{G_{I F}}=0
\end{aligned}
$$


$\rho_{E}+\tilde{d t}_{r G}+m_{f} \times z p d_{w}+\tilde{N}_{E_{I F}}+I S B+\varepsilon_{\Phi E_{F F}}-\tilde{\Phi}_{E_{F F}}=0$

where, $\tilde{P}_{G_{I F}}, \tilde{P}_{G_{I F}(C l / P 1)}, \tilde{P}_{E_{I F}}, \tilde{\Phi}_{G_{I F}}$ and $\tilde{\Phi}_{E_{I F}}$ are the ionosphere-free linear combinations of the pseudorange and carrier-phase observables after applying the above corrections; $z p d_{w}$ is the wet component of the tropospheric zenith path delay; $m_{f}$ troposphere mapping functions.

As indicated earlier, two scenarios are considered when forming the BSSD linear combination, namely a tight and a loose combination. In the first scenario, either a GPS or a Galileo satellite is selected as a reference for both GPS and Galileo observables (Odijk and Teunissen, 2013). Taking a GPS satellite as a reference and using Equations 4.20 to 4.24, we obtain:

$\rho_{G, G}^{i j}+m_{f}^{i j} z p d_{w}+\tilde{\varepsilon}_{P G_{I F}}^{i j}-\tilde{P}_{G_{I F}}^{i j}=0$

$\rho_{G, G}^{i j}+m_{f}^{i j} z p d_{w}+\tilde{\varepsilon}_{P G_{I F}}^{i j}-\tilde{P}_{G_{I F}(C l / P I)}=0$

$\rho_{E, G}^{i k}+m_{f}^{i k} z p d_{w}+I S B+\tilde{\varepsilon}_{P E_{I F}}^{i k}-\tilde{P}_{E G_{I F}}^{i k}=0$

$\rho_{G, G}^{i j}+m_{f}^{i j} z p d_{w}+\tilde{N}_{G_{I F}}^{i j}+\tilde{\varepsilon}_{\Phi G_{I F}}^{i j}-\tilde{\Phi}_{G_{I F}}^{i j}=0$

$\rho_{E, G}^{i k}+m_{f}^{i k} z p d_{w}+I S B+\tilde{N}_{E G_{I F}}^{i k}+\tilde{\varepsilon}_{\Phi E_{I F}}^{i k}-\tilde{\Phi}_{E G_{I F}}^{k}=0$

where, the superscript $i$ refers to the GPS reference satellite; the superscripts $j$ and $k$ refer the GPS and Galileo satellites respectively; $\tilde{N}_{G_{I F}}^{i j}$ and $\tilde{N}_{E G_{I F}}^{i k}$ are given by:

$$
\begin{aligned}
& \tilde{N}_{G_{\text {GF }}}^{i j}=N_{G_{F F}}^{i}-N_{G_{F F}}^{j}+b_{Q \Phi}^{i j}-b_{P}^{i j} \\
& \tilde{N}_{E G_{F F}}^{i k}=N_{G_{F F}}^{i}-N_{E_{F F}}^{k}+b_{r_{E \Phi}}-b_{r_{\Phi}}+b_{E \Phi}^{k}-b_{\Phi}^{i}+b_{P}^{i}-b_{E}^{k}
\end{aligned}
$$

Similarly, when a Galileo satellite is selected as a reference, using Equations 4.20 to 4.24 leads to:

$$
\begin{aligned}
& \rho_{G, E}^{l j}+m_{f}^{l j} z p d_{w}-I S B+\tilde{\varepsilon}_{P G_{I F}^{l j}}^{j j}-\tilde{P}_{G E_{I F}}^{j j}=0 \\
& \rho_{G, E}^{j j}+m_{f}^{l j} z p d_{w}-I S B+\tilde{\varepsilon}_{P G_{I F}}^{j}-\tilde{P}_{G E_{I F}(C I / P 2)}^{j j}=0
\end{aligned}
$$


$\rho_{E, E}^{l k}+m_{f}^{l k} z p d_{w}+\widetilde{\varepsilon}_{P E_{I F}}^{l k}-\tilde{P}_{E_{I F}}^{l k}=0$

$\rho_{G, E}^{l j}+m_{f}^{l j} z p d_{w}-I S B+\tilde{N}_{G E_{F F}}^{l j}+\tilde{\varepsilon}_{\Phi G_{I F}}^{l j}-\tilde{\Phi}_{G E_{I F}}^{j j}=0$

$\rho_{E, E}^{l k}+m_{f}^{l k} z p d_{w}+\tilde{N}_{E_{I F}}^{l k}+\tilde{\varepsilon}_{\Phi E_{I F}}^{l k}-\tilde{\Phi}_{E_{I F}}^{l k}=0$

where, the superscript $l$ refers to the Galileo reference satellite, $\tilde{N}_{G E_{I F}}^{l j}$ and $\tilde{N}_{E_{I F}}^{l k}$ are the BSSD non-integer ambiguity parameters lumped to the receiver and satellite hardware delays, which are given by:

$\tilde{N}_{G E_{I F}}^{j}=N_{E_{I F}}^{l}-N_{G_{I F}}^{j}+b_{r_{Q \Phi}}-b_{r_{E \Phi}}+b_{G \Phi}^{j}-b_{E \Phi}^{l}+b_{E}^{l}-b_{P}^{j}$

$\tilde{N}_{E_{I F}}^{l k}=N_{E_{I F}}^{l}-N_{E_{I F}}^{k}+b_{E \Phi}^{l k}-b_{E}^{l k}$

In the loose BSSD combination, two reference satellites are considered: a GPS reference satellite for the GPS observables and a Galileo satellite for the Galileo observables. Using Equations 4.20 to 4.24 , we obtain:

$\rho_{G, G}^{i j}+m_{f}^{i j} z p d_{w}+\tilde{\varepsilon}_{P G_{I F}}^{i j}-\tilde{P}_{G_{I F}}^{i j}=0$

$\rho_{G, G}^{i j}+m_{f}^{i j} z p d_{w}+\tilde{\varepsilon}_{P G_{I F}}^{i j}-\tilde{P}_{G_{I F}(C I / P 2)}=0$

$\rho_{E, E}^{l k}+m_{f}^{l k} z p d_{w}+\widetilde{\varepsilon}_{P E_{I F}}^{l k}-\tilde{P}_{E_{I F}}^{l k}=0$

$\rho_{G, G}^{i j}+m_{f}^{i j} z p d_{w}+\tilde{N}_{G_{I F}}^{i j}+\tilde{\varepsilon}_{\Phi G_{I F}}^{i j}-\tilde{\Phi}_{G_{I F}}^{i j}=0$

$\rho_{E, E}^{l k}+m_{f}^{l k} z p d_{w}+\tilde{N}_{E_{I F}}^{l k}+\tilde{\varepsilon}_{\Phi E_{I F}}^{l k}-\tilde{\Phi}_{E_{I F}}^{l k}=0$

where, $\tilde{N}_{G_{I F}}^{l j}$ and $\tilde{N}_{E_{I F}}^{l k}$ are the BSSD non-integer ambiguity parameters lumped to the receiver and satellite hardware delays as shown in Equations 4.44 and 4.45.

$$
\begin{aligned}
& \tilde{N}_{G_{I F}^{j j}}^{j^{j}}=N_{G_{I F}}^{i}-N_{G_{I F}}^{j}+b_{G \Phi}^{i j}-b_{P}^{i j} \\
& \tilde{N}_{E_{\mathbb{F}}}^{k}=N_{E_{\mathbb{F}}}^{k}-N_{E_{\mathbb{F}}}^{k}+b_{E \Phi}^{l k}-b_{E}^{l k}
\end{aligned}
$$




\subsection{LEAST SQUARES ESTIMATION TECHNIQUE}

Under the assumption that the observations are uncorrelated and the errors are normally distributed with zero mean, the covariance matrix of the un-differenced observations takes the form of a diagonal matrix. The elements along the diagonal line represent the variances of the code and carrier phase measurements. In our solution, we consider that the ratio between the standard deviation of the code and carrier-phase measurements to be 100. When forming BSSD, however, the differenced observations become mathematically correlated. This leads to a fully populated covariance matrix at a particular epoch.

The general linearized form for the above observation equations around the initial (approximate) vector $\boldsymbol{u}^{0}$ and observables $l$ can be written in a compact form as:

$$
\boldsymbol{f}(\boldsymbol{u}, l)=A \backslash u-w-r=0
$$

where $\boldsymbol{u}$ is the vector of unknown parameters; $\boldsymbol{A}$ is the design matrix, which includes the partial derivatives of the observation equations with respect to the unknown parameters $\boldsymbol{u} ; \boldsymbol{\Delta} \boldsymbol{u}$ is the unknown vector of corrections to the approximate parameters $\boldsymbol{u}^{0}$, i.e., $\boldsymbol{u}=\boldsymbol{u}^{0}+\boldsymbol{\Delta} \boldsymbol{u} ; \boldsymbol{w}$ is the misclosure vector and $\boldsymbol{r}$ is the vector of residuals. The sequential least-squares solution for the unknown parameters $\boldsymbol{\Delta} \boldsymbol{u}_{\boldsymbol{i}}$ at an epoch $i$ can be obtained from (Vanicek and Krakiwsky, 1986):

$$
\begin{aligned}
& \Delta \boldsymbol{u}_{i}=\Delta \boldsymbol{u}_{i-1}+\boldsymbol{M}_{i-1}^{-1} \boldsymbol{A}_{i}^{T}\left(\boldsymbol{C}_{l_{i}}+\boldsymbol{A}_{i} \boldsymbol{M}_{i-1}^{-1} \boldsymbol{A}_{i}^{T}\right)^{-1}\left[\boldsymbol{w}_{i}-\boldsymbol{A}_{i} \Delta \boldsymbol{u}_{i-1}\right] \\
& \boldsymbol{M}_{i}^{-1}=\boldsymbol{M}_{i-1}^{-1}-\boldsymbol{M}_{i-1}^{-1} \boldsymbol{A}_{i}^{T}\left(\boldsymbol{C}_{l_{i}}+\boldsymbol{A}_{i} \boldsymbol{M}_{i-1}^{-1} \boldsymbol{A}^{T}\right)^{-1} \boldsymbol{A}_{i} \boldsymbol{M}_{i-1}^{-1} \\
& \boldsymbol{C}_{\Delta u_{i}}=\boldsymbol{M}_{i}^{-1}=\boldsymbol{M}_{i-1}^{-1}-\boldsymbol{M}_{i-1}^{-1} \boldsymbol{A}_{i}^{T}\left(\boldsymbol{C}_{l_{i}}+\boldsymbol{A}_{i} \boldsymbol{M}_{i-1}^{-1} \boldsymbol{A}^{T}\right)^{-1} \boldsymbol{A}_{i} \boldsymbol{M}_{i-1}^{-1}
\end{aligned}
$$

where $\boldsymbol{\Delta} \boldsymbol{u}_{i-1}$ is the least-squares solution for the estimated parameters at epoch $i-1 ; \boldsymbol{M}$ is the matrix of the normal equations; $\boldsymbol{C}_{l}$ and $\boldsymbol{C}_{\Delta u}$ are the covariance matrices of the observations and unknown parameters, respectively. It should be pointed out that the usual batch least-squares adjustment should be used in the first epoch, i.e., for $i=1$. The batch solution for the estimated parameters and the inverse of the normal equation matrix are given, respectively, by (Vanicek and Krakiwsky, 1986): 


$$
\begin{aligned}
& \Delta \boldsymbol{u}_{l}=\left[\boldsymbol{C}_{x^{0}}^{-1}+\boldsymbol{A}_{l}^{T} \boldsymbol{C}_{l_{1}}^{-1} \boldsymbol{A}_{l}\right]^{-1} \boldsymbol{A}_{l}^{T} \boldsymbol{C}_{l_{1}}^{-l} \boldsymbol{w}_{l} \\
& \boldsymbol{M}_{1}^{-1}=\left[\boldsymbol{C}_{x^{0}}^{-1}+\boldsymbol{A}_{l}^{T} \boldsymbol{C}_{l_{1}}^{-1} \boldsymbol{A}_{l}\right]^{-1}
\end{aligned}
$$

where $\boldsymbol{C}_{x}{ }^{0}$ is a priori covariance matrix for the approximate values of the unknown parameters. It should be pointed out that, as the IGS-MEGX precise orbits refer to the IGS08, the resulting station coordinates will be referenced to the IGS08.

When a GPS satellite is selected as a reference to form the BSSD for both GPS and Galileo observations, the design matrix $\boldsymbol{A}$ and the vector of corrections $\boldsymbol{\Delta} \boldsymbol{u}$ take the form:

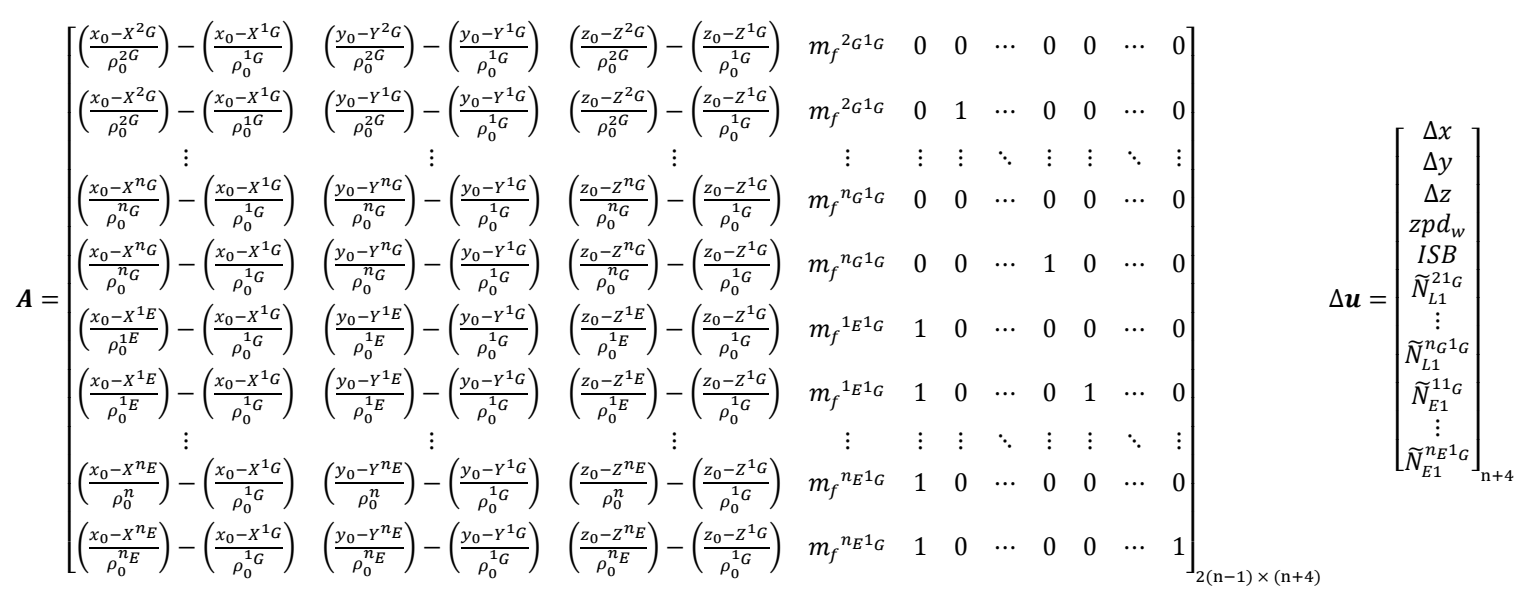

where ' $l_{G}$ ' refers to the GPS reference satellite; $n_{G}$ refers to the number of visible GPS satellites; $n_{E}$ refers to the number of visible Galileo satellites; $n=n_{G}+n_{E}$ is the total number of the observed satellites for both GPS and Galileo systems; $x_{0}, y_{0}$ and $z_{0}$ are the approximate receiver coordinates; $X^{j_{G}}, Y^{j_{G}}, Z^{j_{G}}, j=1,2, \ldots, n_{G}$ are the known GPS satellite coordinates; $X^{k_{E}}, Y^{k_{E}}, Z^{k_{E}}, k=1,2, \ldots, n_{E}$ are the known Galileo satellite coordinates; $\rho_{0}$ is the approximate receiver-satellite range. The unknown parameters in the above system are the corrections to the receiver coordinates, $\Delta x, \Delta y$, and $\Delta z$, the wet component of the tropospheric zenith path delay $z p d_{w}$, the inter-system bias $I S B$, and the non-integer ambiguity parameters $\widetilde{N}$.

The advantage of the above system (4.52) is that the number of unknown parameters is reduced by two (i.e., becomes $n_{G}+n_{E}+4$ ), in comparison with the un-differenced PPP counterpart. This, however, comes at the expense of reducing the number of BSSD observation equations to $2\left(n_{G}+n_{E}-1\right)$. As such, the redundancy remains unchanged and equals $n_{G}+n_{E}-6$. Similarly, we need 
a minimum of two Galileo satellites in order to contribute to the solution. By analogy, the use of a Galileo satellite as a reference to form the BSSD for both GPS and Galileo observations leads to:

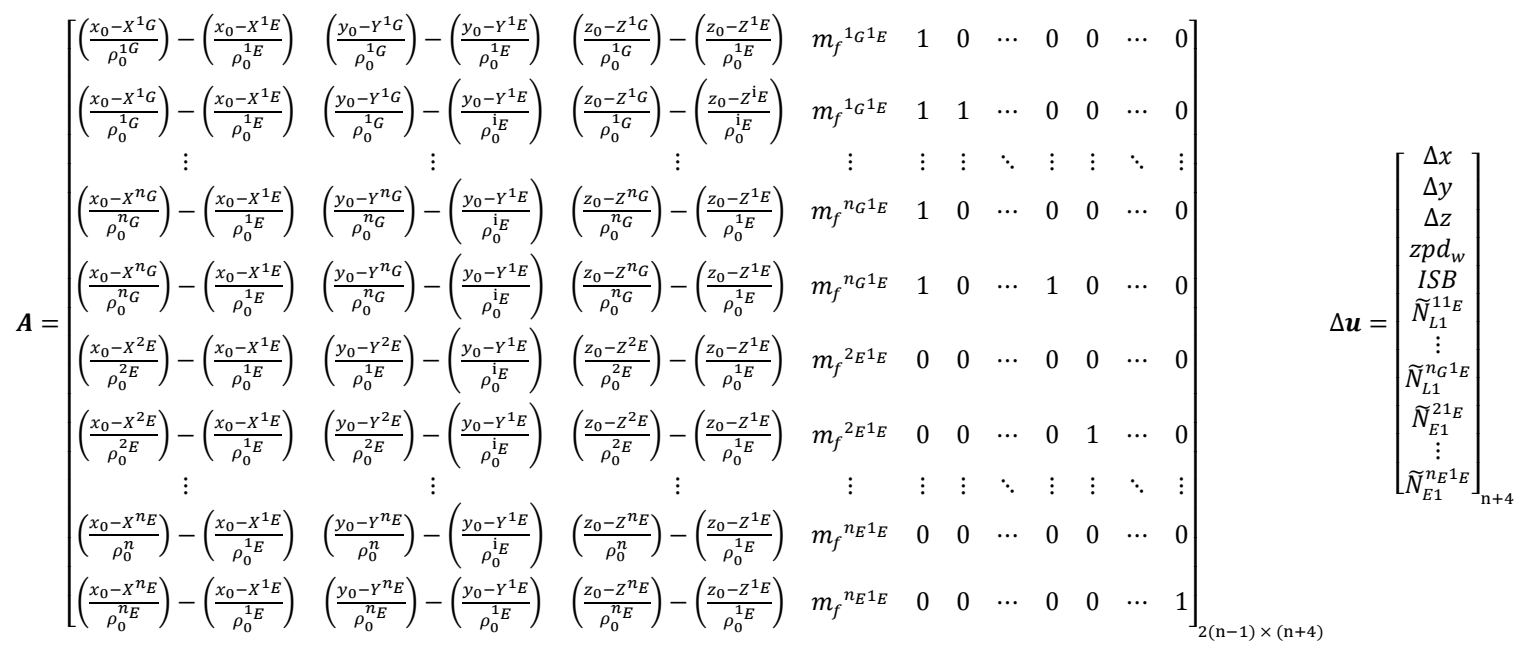

where $l_{E}$ refers to the Galileo reference satellite. All other parameters are as defined above. Similar to the above BSSD scenario, the redundancy remains unchanged and equals $n_{G}+n_{E}-6$.

When two reference satellites are selected to form the BSSD, i.e., per-constellation BSSD, the design matrix $\boldsymbol{A}$ and the vector of corrections $\boldsymbol{\Delta} \boldsymbol{u}$ take the form:

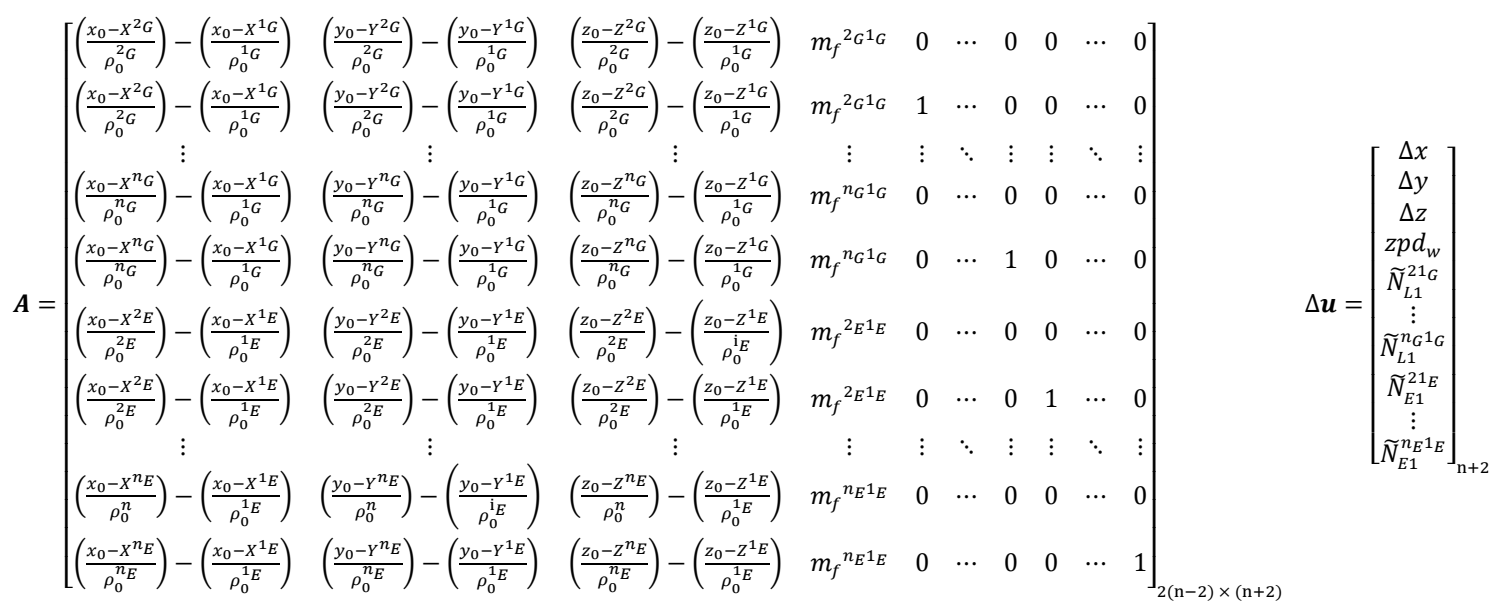

The major advantage of the above per-constellation (or loose combination) system is that the modified receiver clock error and the inter-system bias are cancelled out. In addition, the total number of unknown parameters is reduced by 4 to become $n_{G}+n_{E}+2$, in comparison with the undifferenced scenario. This, however, comes at the expense of reducing the number of BSSD 
observation equations to $2\left(n_{G}+n_{E}-2\right)$. This means that the redundancy remains unchanged and equals $n_{G}+n_{E}-6$. Similar to the previous scenarios, the redundancy for the GPS-only scenario is still $n_{G}-5$ and the addition of Galileo satellites increased the overall redundancy to $n_{E}-1$. As such, we still need a minimum of two Galileo satellites in order to contribute to the solution.

\subsection{RESULTS AND DISCUSSIONS}

To verify the developed GPS/Galileo PPP model, GPS and Galileo measurements at six welldistributed stations (Figure 4.1) were selected from the IGS tracking network (Dow et al. 2009). Those stations are occupied by GNSS receivers, which are capable of simultaneously tracking the GPS and Galileo constellations. A total of 12 data sets representing the GPS/Galileo measurements over two different days, namely January 1, 2014 and July 8, 2014, are processed. Only one hour of each day with four Galileo satellites of each data set is considered in our analysis. All data sets have a sampling rate of 30 seconds.

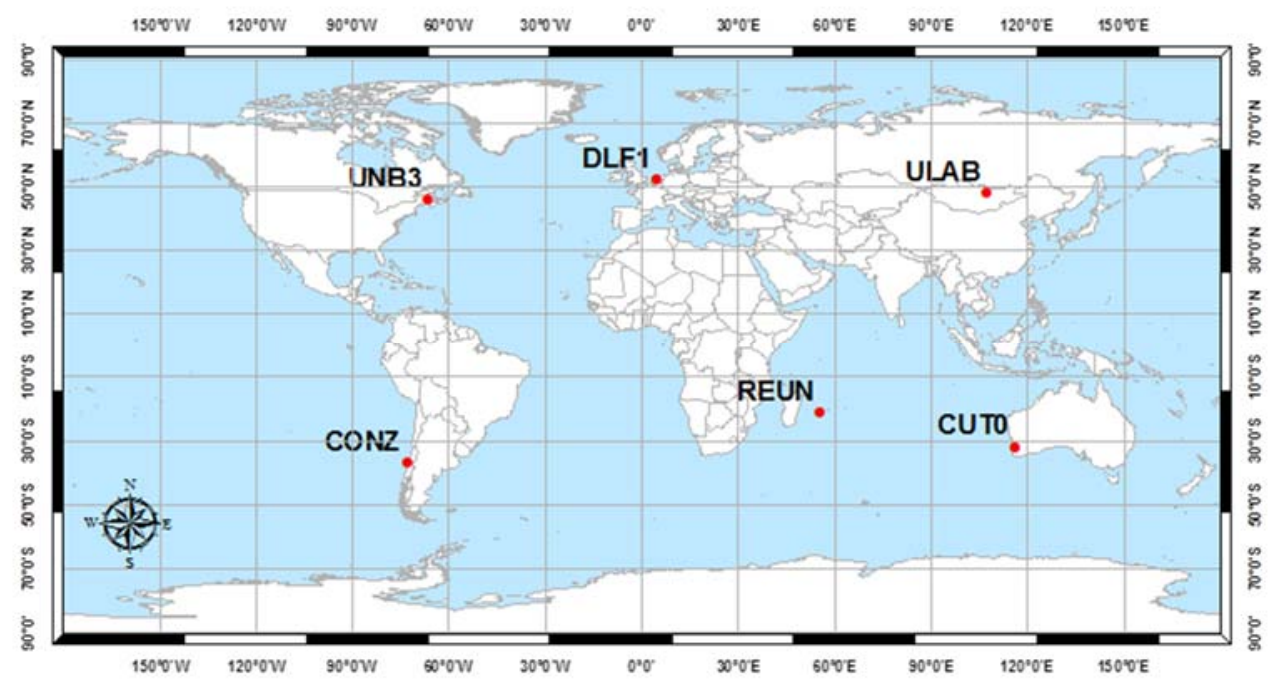

Figure 4.1 Analysis stations

The positioning results for stations CONZ, and CUT0 (January 1, 2014) and stations DLF1, and UNB3 (July 8, 2014) are presented below. Similar results are obtained for the other stations. However, a summary of the convergence times and the three-dimensional PPP solution standard deviations are presented below for all stations. 
Natural Resources Canada's GPSPace PPP software was modified to handle data from both GPS and Galileo systems, which enables a combined GPS/Galileo PPP solution as detailed above. In addition to our PPP GPS/Galileo BSSD model, we also obtained the solutions of the traditional un-differenced ionosphere-free GPS-only and GPS/Galileo PPP models. The latter results were used to assess the performance of our newly developed PPP model. Figures 2 to 4 summarize the positioning results in the East, North, and Up directions, respectively, for all analysis modes. As can be seen, the un-differenced GPS-only PPP solution indicates that the model is capable of obtaining a sub-decimetre level accuracy. However the solution takes about 20 minutes to converge to decimetre level accuracy. On the other hand, the addition of Galileo observations reduces the convergence of the un-differenced PPP solution to about 15 minutes.

Considering the first scenario (i.e., tight combination) of the newly developed ionosphere-free BSSD GPS/Galileo PPP model, we processed all data sets twice: the first considers a GPS satellite as a reference for both GPS and Galileo observables, while the second considers a Galileo satellite as a reference. As can be seen in Figures 4.2 to 4.4, almost identical positioning results are obtained with an average convergence time equals 10 minutes. We also processed the data sets through the loose combination PPP model and obtained a comparable convergence time to the first scenario. However, the performance of the loose combination model was better than that of the tight combination model within the convergence interval. In comparison with the GPS-only PPP model, the newly developed BSSD model improved the PPP solution convergence by about 50\%. Figures 4.5 and 4.6 summarize the convergence times for all analysis cases, which confirm the PPP solution consistency at all stations.
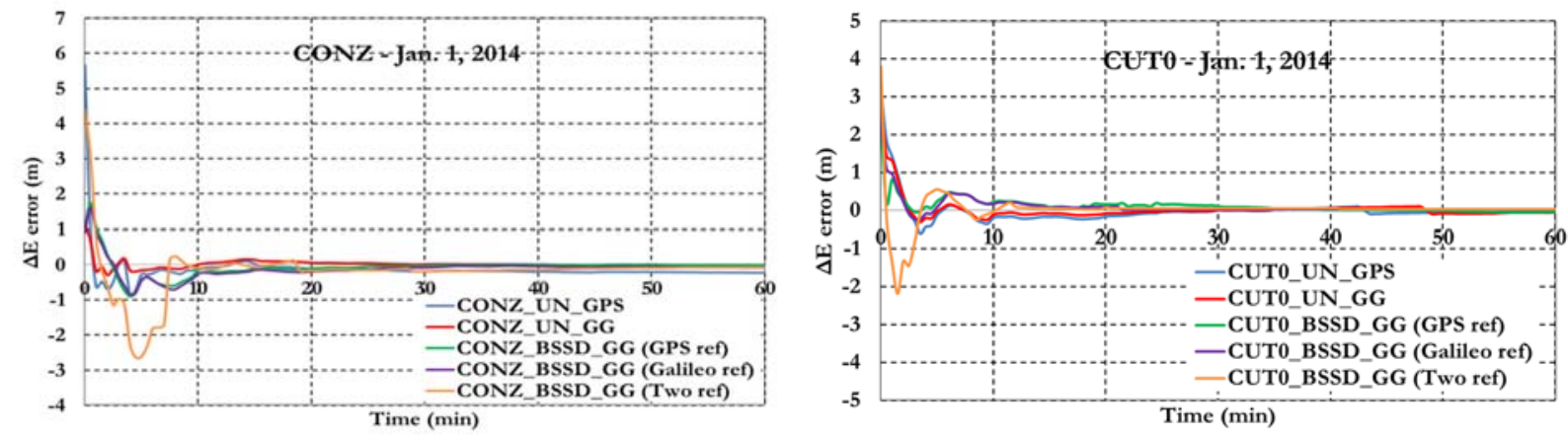

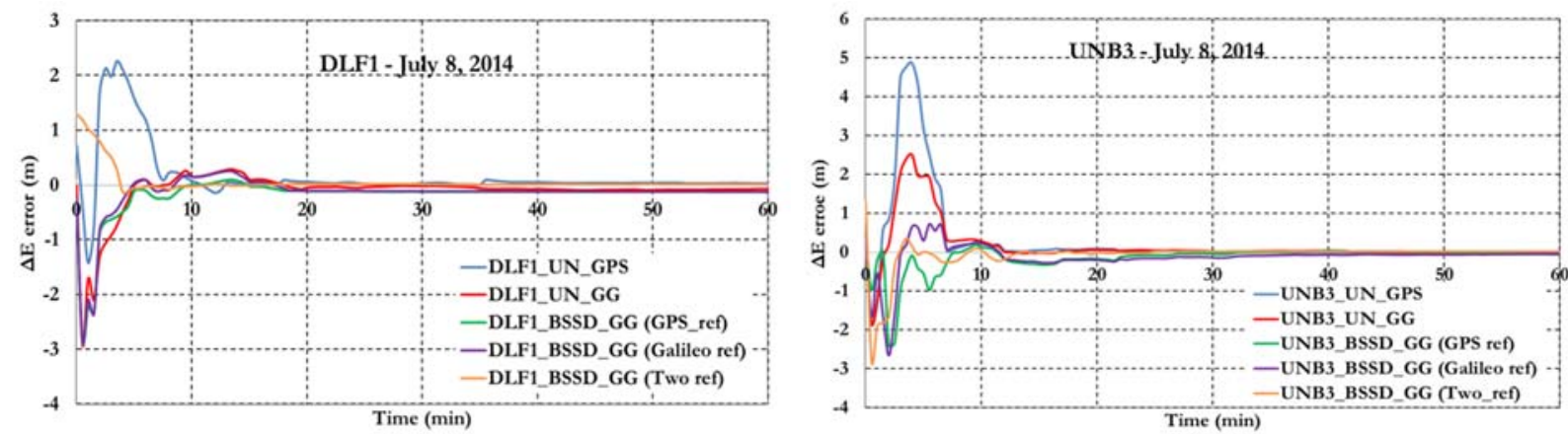

Figure 4.2 Positioning errors in East direction
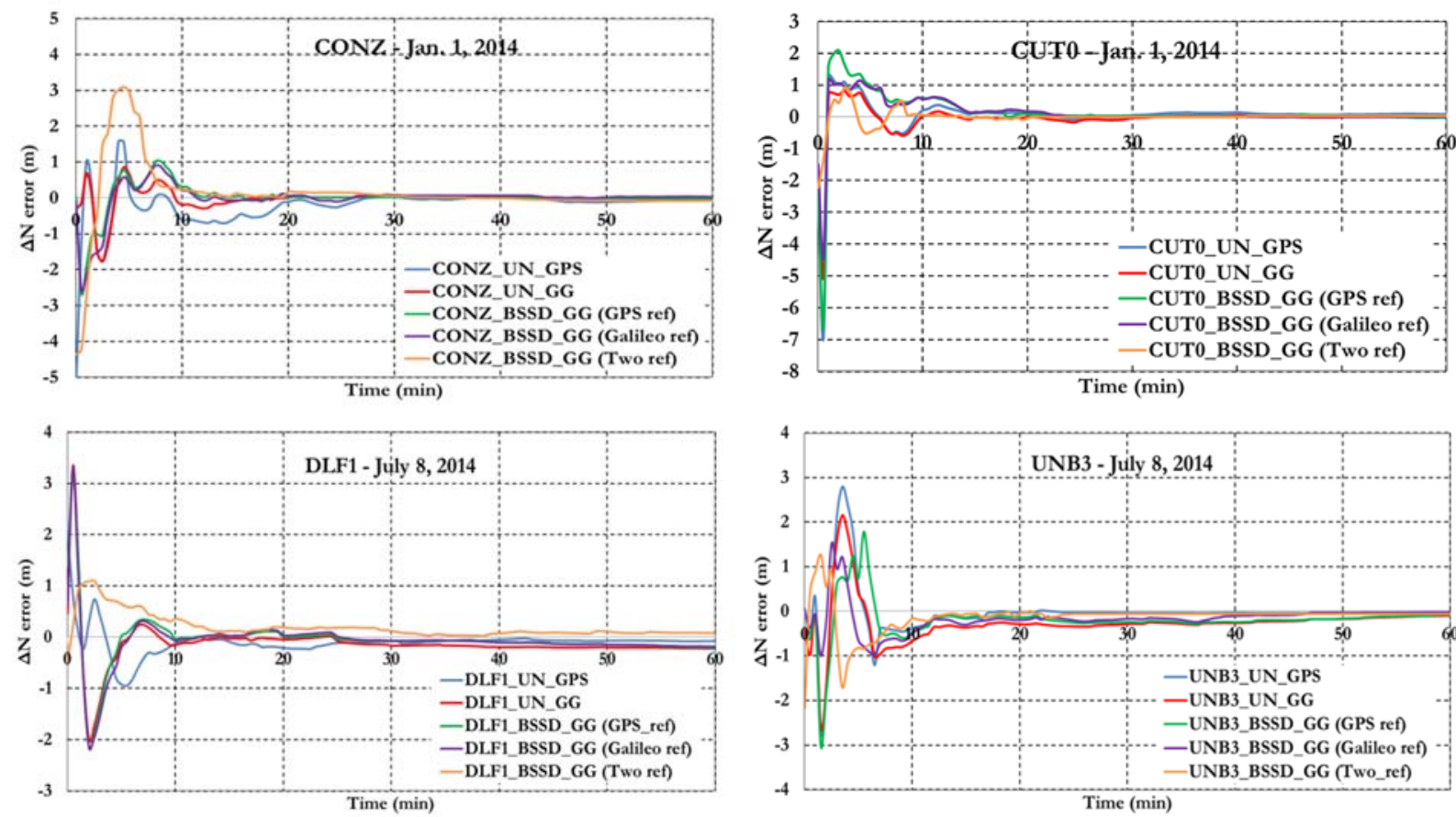

Figure 4.3 Positioning errors in North direction
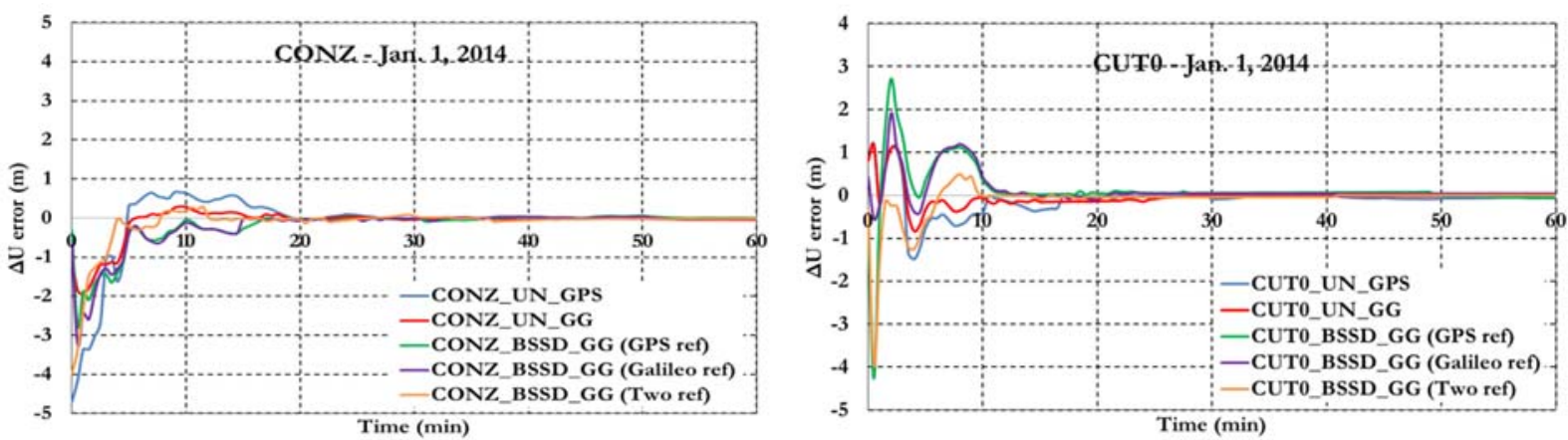

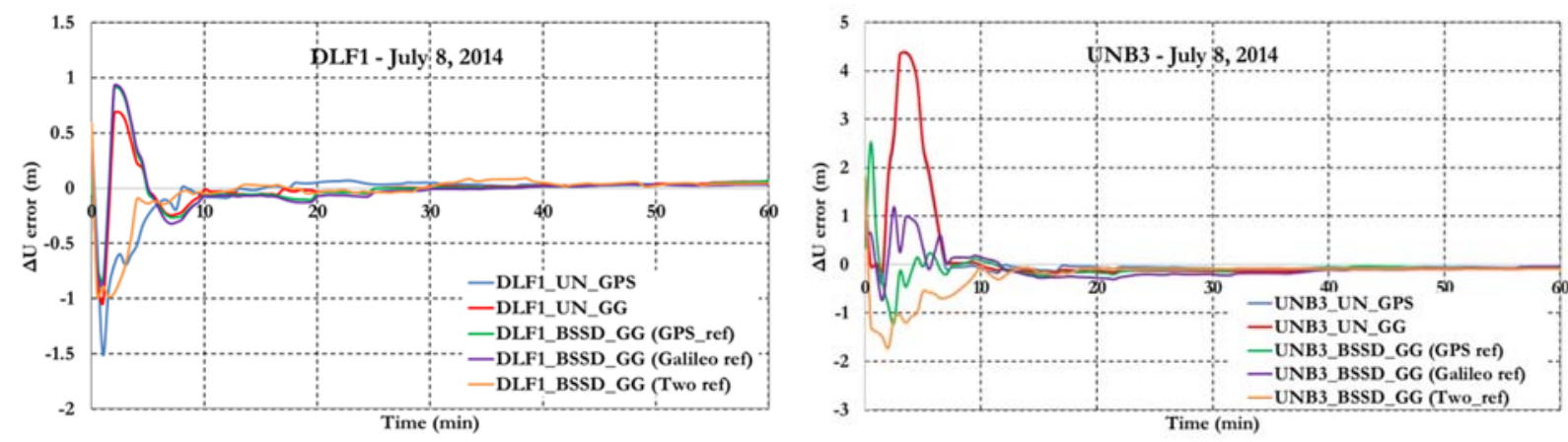

Figure 4.4 Positioning errors in Up direction

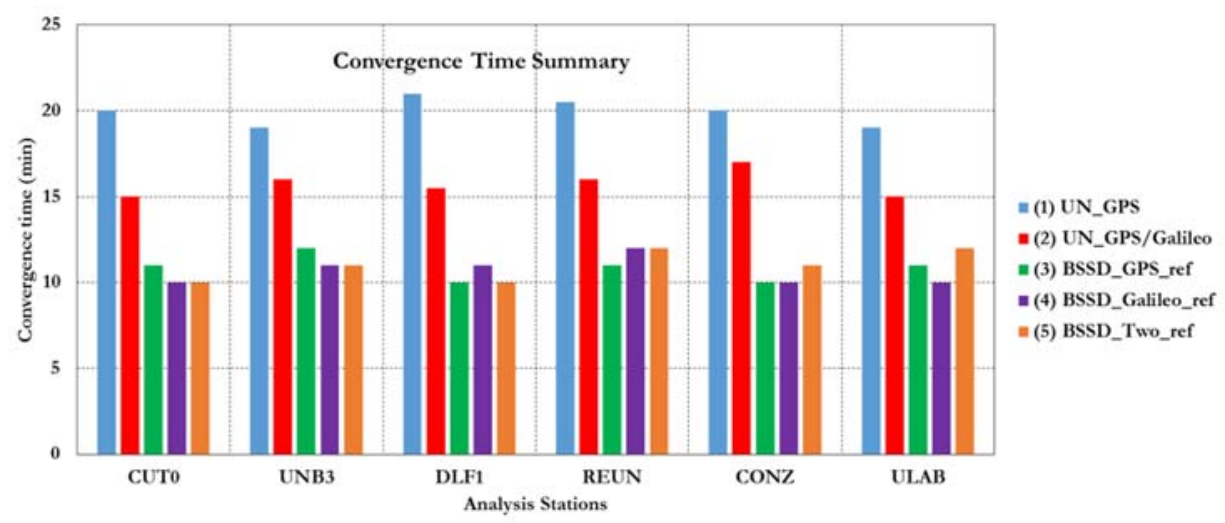

Figure 4.5 Summary of convergence times of all stations and analysis cases. (1) Un-differenced GPS model; (2) Un-differenced GPS/Galileo model; (3) BSSD model with a GPS satellite as a reference; (4) BSSD model with a Galileo satellite as a reference; (5) BSSD model with both a GPS and a Galileo satellite as reference satellites

To further assess the performance of the various PPP models, the solution output is sampled every 10 minutes and the standard deviation of the computed station coordinates is calculated for each sample. Figure 4.6 shows the position standard deviations in the East, North, and Up directions, respectively. Examining the standard deviations after 10 minutes, it can be seen that the newly developed GPS/Galileo PPP model improves the precision of the estimated parameters by about $50 \%$ and $25 \%$, in comparison with the un-differenced GPS-only model, when the loose and the tight combinations are used, respectively. As the number of epochs, and consequently the number of measurements, increases the performance of the various models tends to be comparable. An exception, however, is the loose combination model, which is found superior to all other PPP models. 

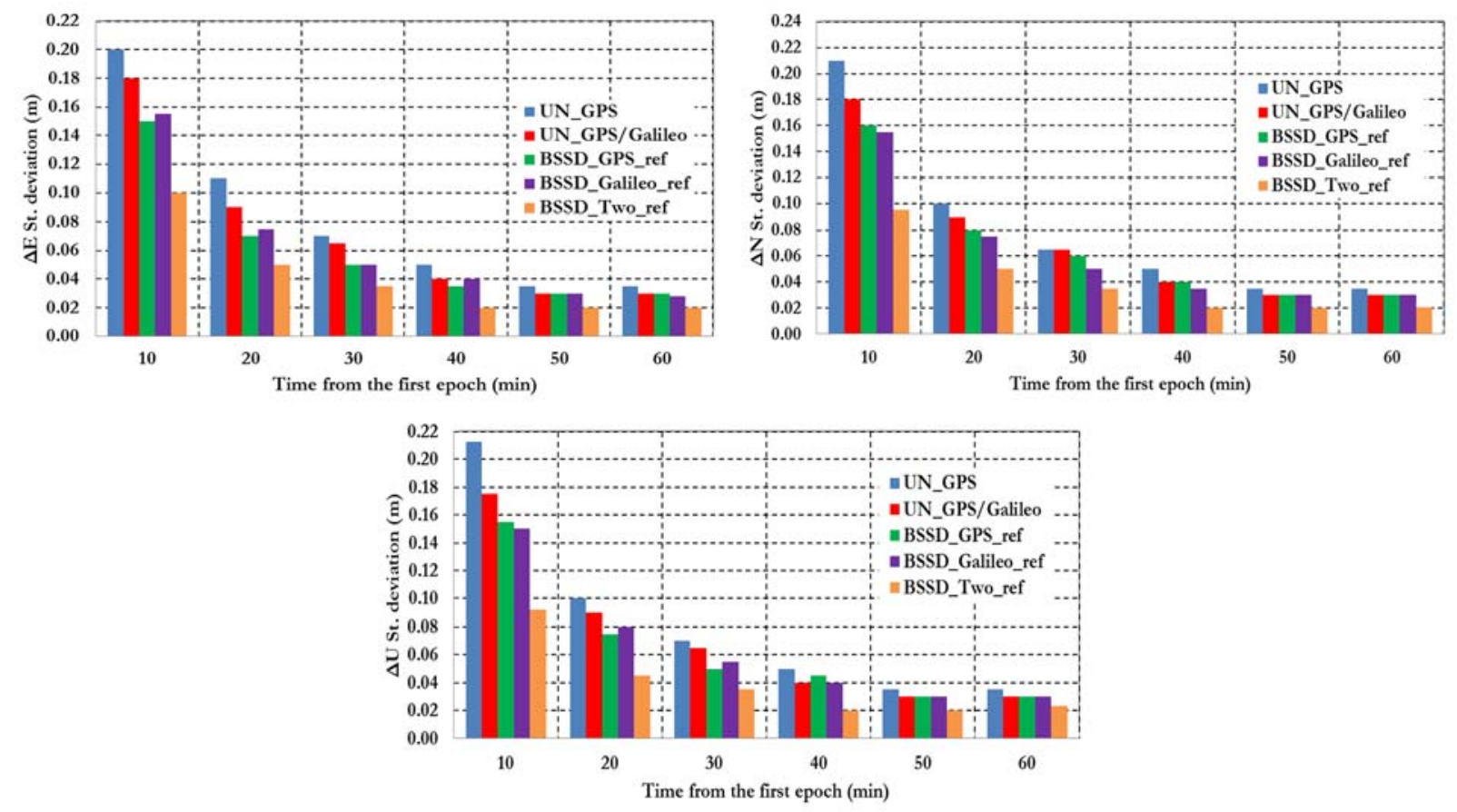

Figure 4.6 Positioning standard deviations in East, North, and Up directions

\subsection{CONCLUSIONS}

This paper presented a PPP model, which combines GPS and Galileo observations in BSSD mode. Two scenarios have been considered when forming BSSD, namely loose and tight combinations. It has been shown that the newly developed PPP model improves the solution convergence time by about 50\%, in comparison with the un-differenced GPS PPP model, regardless of the type of BSSD combination used. In addition, the newly developed model improves the precision of the estimated parameters by about $50 \%$ and $25 \%$, in comparison with the un-differenced GPS-only model, when the loose and the tight combinations are used, respectively. As the number of epochs increases, the performance of the various models tends to be comparable. An exception, however, is the loose combination model, which is found superior to all other PPP models. Almost identical results are obtained through the tight combination when either a GPS or a Galileo satellite is selected as a reference. 


\section{CHAPTER 5}

\section{PERFORMANCE ANALYSIS OF SEVERAL GPS/GALILEO PRECISE POINT POSITIONING MODELS}

This chapter examines the performance of several precise point positioning (PPP) models, which combine dual-frequency GPS/Galileo observations in the un-differenced and between-satellite single-difference (BSSD) modes. These include the traditional un-differenced model, the decoupled clock model, the semi-decoupled clock model, and between-satellite single-difference model. The results were published as:

Afifi, A., El-Rabbany, A. (2015). Performance analysis of several GPS/Galileo precise point positioning models, Sensors, 15(6), 14701-14726; doi:10.3390/s150614701

Modifications to the original manuscript were made only for proper identification of sections, figures and tables, as well as to assure the uniformity of symbol and equation notation throughout this dissertation. 


\begin{abstract}
This paper examines the performance of several precise point positioning (PPP) models, which combine dual-frequency GPS/Galileo observations in the un-differenced and between-satellite single-difference (BSSD) modes. These include the traditional un-differenced model, the decoupled clock model, the semi-decoupled clock model, and the between-satellite singledifference model. We take advantage of the IGS-MGEX network products to correct for the satellite differential code biases and the orbital and satellite clock errors. Natural Resources Canada's GPSPace PPP software is modified to handle the various GPS/Galileo PPP models. A total of six data sets of GPS and Galileo observations at six IGS stations are processed to examine the performance of the various PPP models. It is shown that the traditional undifferenced GPS/Galileo PPP model, the GPS decoupled clock model, and the semi-decoupled clock GPS/Galileo PPP model improve the convergence time by about $25 \%$ in comparison with the un-differenced GPS-only model. In addition, the semi-decoupled GPS/Galileo PPP model improves the solution precision by about $25 \%$ compared to the traditional un-differenced GPS/Galileo PPP model. Moreover, the BSSD GPS/Galileo PPP model improves the solution convergence time by about 50\%, in comparison with the un-differenced GPS PPP model, regardless of the type of BSSD combination used. As well, the BSSD model improves the precision of the estimated parameters by about $50 \%$ and $25 \%$ when the loose and the tight combinations are used, respectively, in comparison with the un-differenced GPS-only model. Comparable results are obtained through the tight combination when either a GPS or a Galileo satellite is selected as a reference.
\end{abstract}

\title{
5.1. INTRODUCTION
}

GNSS precise point positioning (PPP) has proven to be capable of providing positioning accuracy at the sub-decimeter and decimeter levels in static and kinematic modes, respectively. PPP accuracy and convergence time are controlled by the ability to mitigate all potential error biases in the system. Several comprehensive studies have been published on the accuracy and convergence time of un-differenced GPS and GPS/Galileo PPP models (see for example, Zumberge et al, 1997; Kouba and Héroux, 2001; Colombo et al., 2004; Ge at el., 2008; Collins at al., 2010; Afifi and El-Rabbany, 2015). In the traditional un-differenced GPS PPP model, because of the presence of the un-calibrated hardware delays, the ambiguity parameters are 
typically obtained as real-valued numbers (Collins at al., 2010; Ge et al., 2008; Mercier and Laurichesse, 2008; Geng et al., 2010a). This in turn affects the GPS PPP solution convergence and accuracy (Geng et. al., 2010b). However, recent research has demonstrated that the correct integer values for the ambiguity parameters can be recovered if the satellite hardware delays can be calibrated. Figures 5.1 and 5.2 show the IGS average estimated values of the receiver and satellite differential code biases, respectively, for 2014 (IGS, 2015). As can be seen in Figure 5.2, Galileo satellite differential code biases of E1/E5a signals are relatively smaller than the GPS L1/L2 counterpart.
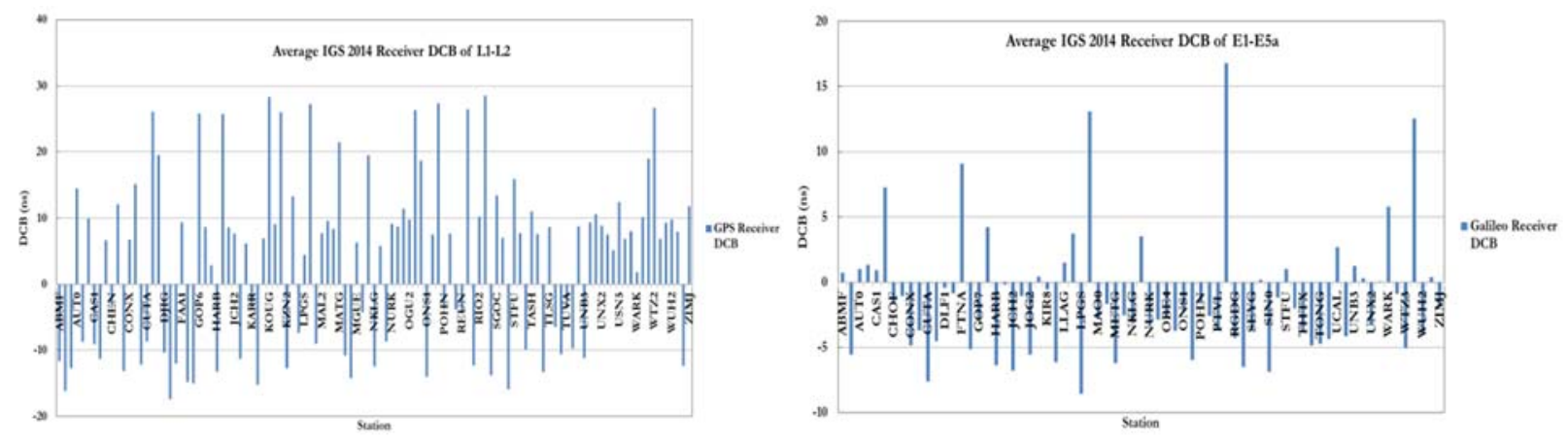

Figure 5. 1 Average 2014 IGS receiver DCB for GPS and Galileo signals

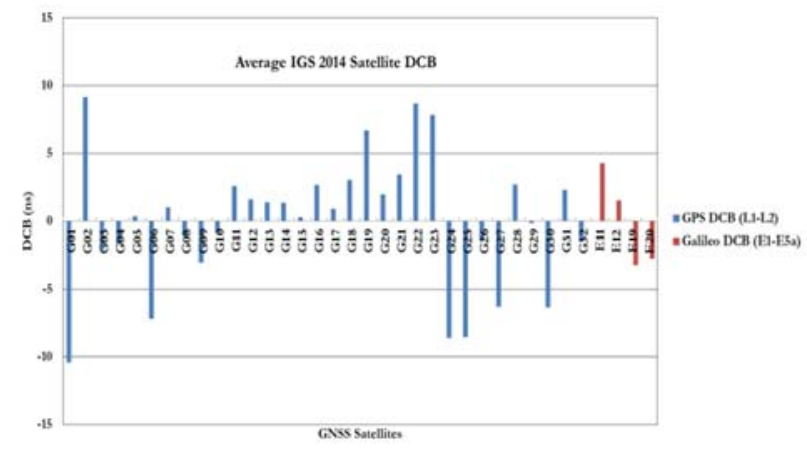

Figure 5. 2 Average IGS 2014 satellite DCB for both GPS/Galileo signals

For a single GNSS constellation, between-satellite single-difference (BSSD) linear combination cancels out all receiver-related errors, including the receiver hardware delays, which significantly improves the convergence time (Afifi and El-Rabbany, 2014; Elsobeiey and ElRabbany, 2014; Ge et al., 2008; Colombo et al., 2004). This, however, is not the case when the measurements of two or more constellations are combined. When forming BSSD for GPS and 
Galileo measurements, three scenarios can be considered on the selection of the reference satellite. Either a GPS or a Galileo satellite is selected as a reference for both GPS and Galileo observables. Alternatively, two reference satellites are selected: a GPS reference satellite for the GPS observables and a Galileo satellite for the Galileo observables. The first approach is commonly referred to as tight combination, while the latter is commonly referred to as perconstellation or loose combination (Afifi and El-Rabbany, 2015).

This paper examines the performance of several PPP models, which combine the dual-frequency GPS/Galileo observables in both un-differenced and BSSD mode. The IGS-MGEX network products used to correct for the satellite differential code biases, the orbital and satellite clock errors (Montenbruck et al., 2014a). As the IGS-MGEX products are presently referenced to the GPS time and since we use mixed GNSS receivers that also use the GPS time as a reference, the GPS to Galileo time offset (GGTO) is cancelled out in our models. The inter-system bias is either cancelled out through differencing the observations or is treated as an additional unknown parameter. The Hopfield tropospheric correction model is used, along with the Vienna mapping function, to account for the hydrostatic component of the tropospheric delay (Hopfield, 1972; Boehm and Schuh, 2004). The wet component is treated as an additional unknown parameter in the estimation model. Other corrections are also applied, including the effect of ocean loading (Bos and Scherneck, 2014; IERS, 2010), Earth tide (Kouba, 2009), carrier-phase windup (Leick, 2004; Wu et al., 1993), Sagnac (Kaplan and Heagarty, 2006), relativity (Hofmann-Wellenhof et al., 2008), and satellite and receiver antenna phase-center variations (Dow et al. 2009). Natural Resources Canada's GPSPace PPP software is modified to handle the various GPS/Galileo PPP models. A total of 6 data sets of GPS and Galileo observations at six IGS stations are processed to examine the performance of the various PPP models. It is shown that the traditional undifferenced GPS/Galileo PPP model, the GPS decoupled clock model, and semi-decoupled clock GPS/Galileo PPP model improve the convergence time by about $25 \%$ in comparison with the undifferenced GPS-only model. In addition, the semi-decoupled GPS/Galileo PPP model improves the solution precision by about 25\% compared to the traditional un-differenced GPS/Galileo PPP model. Moreover, the BSSD GPS/Galileo PPP model improves the solution convergence time by about 50\%, in comparison with the un-differenced GPS PPP model, regardless of the type of BSSD combination used. As well, the BSSD model improves the precision of the estimated 
parameters by about $50 \%$ and $25 \%$ when the loose and the tight combinations are used, respectively, in comparison with the un-differenced GPS-only model. Comparable results are obtained through the tight combination when either a GPS or a Galileo satellite is selected as a reference.

\subsection{UN-DIFFERENCED GPS/GALILEO PPP MODELS}

\subsubsection{TRADITIONAL GPS/GALILEO PPP MODEL}

PPP has traditionally been carried out using dual-frequency ionosphere-free linear combinations of carrier-phase and pseudorange GPS measurements. Equations 5.1 to 5.4 show the ionosphere free linear combination of both GPS/Galileo observations (Afifi and El-Rabbany, 2015).

$$
\begin{aligned}
& P_{G_{I F}}=\rho_{G}+c\left[d t_{r G}-d t^{s}\right]+c\left[\alpha d_{P 1}-\beta d_{P 2}\right]_{r}+c\left[\alpha d_{P 1}-\beta d_{P 2}\right]^{s}+T_{G}+\varepsilon_{P G_{I F}} \\
& P_{E_{I F}}=\rho_{E}+c\left[d t_{r G}-G G T O-d t^{s}\right]+c\left[\alpha d_{E 1}-\beta d_{E 5 a}\right]_{r}+c\left[\alpha d_{E 1}-\beta d_{E 5 a 2}\right]^{s}+T_{E}+\varepsilon_{E_{I F}} \\
& \Phi_{G_{I F}}=\rho_{G}+c\left[d t_{r G}-d t^{s}\right]+c\left[\alpha \delta_{L 1}-\beta \delta_{L 2}\right]_{r}+c\left[\alpha \delta_{L 1}-\beta \delta_{L 2}\right]^{s}+T_{G}+N_{G_{I F}}+\phi_{r 0_{G_{I F}}}+\phi_{0_{G_{I F}}^{s}}+\varepsilon_{\Phi G_{I F}} \\
& \Phi_{E_{I F}}=\rho_{E}+c\left[d t_{r G}-G G T O-d t^{s}\right]+c\left[\alpha \delta_{E 1}-\beta \delta_{E 5 a}\right]_{r}+c\left[\alpha \delta_{E l}-\beta \delta_{E 5 a}\right]^{s}+T_{E}+N_{E_{I F}}+\phi_{r 0_{E F}}+\phi_{0_{E_{I F}}^{s}}+\varepsilon_{\Phi E_{I F}}
\end{aligned}
$$

where the subscripts $\mathrm{G}$ and $\mathrm{E}$ refer to the GPS and Galileo satellite systems, respectively; $P_{G_{I F}}$ and $P_{E_{I F}}$ are the ionosphere-free pseudorange in meters for GPS and Galileo systems, respectively; $\Phi_{G_{I F}}$ and $\Phi_{E_{I F}}$ are the ionosphere-free carrier phase measurements in meters for GPS and Galileo systems, respectively; $\rho$ is the true geometric range from receiver at reception time to satellite at transmission time in meter; $d t r, d t^{s}$ are the clock errors in seconds for the receiver at signal reception time and the satellite at signal transmission time, respectively; $d_{P 1 r}, d_{P 2 r}, d_{E 1 r}$, $d_{E 5 a r}$ are frequency-dependent code hardware delays for the receiver at reception time in seconds; $d_{P 1}{ }^{s}, d_{P 2}{ }^{s}, d_{E 1}{ }^{S}, d_{E 5 a}{ }^{s}$ are frequency-dependent code hardware delays for the satellite at transmission time in seconds; $\delta_{L 1}, \delta_{L 2}, \delta_{E 1}, \delta_{E 5 a}$ are frequency-dependent carrier-phase hardware delays for the receiver at reception time in seconds; $\delta_{L 1}{ }^{S}, \delta_{L 2}{ }^{S}, \delta_{E 1}{ }^{S}, \delta_{E 5 a}{ }^{S}$ are 
frequency-dependent carrier-phase hardware delays for the satellite at transmission time in seconds; $T$ is the tropospheric delay in meter; $N_{G_{I F}}, N_{E_{I F}}$ are the ionosphere-free linear combinations of the ambiguity parameters for both GPS and Galileo carrier-phase measurements in meters, respectively (Equations 5.5 and 5.6); $\phi_{r 0_{G_{I F}}}, \phi_{0_{G_{I F}}^{S}}, \phi_{r 0_{E_{I F}}}, \phi_{0_{E_{I F}}^{S}}$ are ionosphere-free linear combinations of frequency-dependent initial fractional phase biases in the receiver and satellite channels for both GPS and Galileo in meters, respectively; $c$ is the speed of light in vacuum in meter per second; $\varepsilon_{P_{I F}}, \varepsilon_{E_{I F}}, \quad \varepsilon_{\Phi G_{I F}}, \varepsilon_{\Phi E_{I F}}$ are the ionosphere-free linear combinations of the relevant noise and un-modeled errors in meter; $\alpha_{G}, \beta_{G}, \alpha_{E}, \beta_{E}$ are the ionosphere-free linear combination coefficients for both GPS and Galileo, which are given, respectively, by: $\alpha_{G}=\frac{f_{1}^{2}}{f_{1}^{2}-f_{2}^{2}}, \beta_{G}=\frac{f_{2}^{2}}{f_{1}^{2}-f_{2}^{2}}, \alpha_{E}=\frac{f_{E 1}^{2}}{f_{E 1}^{2}-f_{E S a}^{2}}, \beta_{E}=\frac{f_{E s a}^{2}}{f_{E 1}^{2}-f_{E S a}^{2}}$.

where $f_{1}$ and $f_{2}$ are GPS $\mathrm{L}_{1}$ and $\mathrm{L}_{2}$ signals frequencies; $f_{E I}$ and $f_{E 5 a}$ are Galileo $\mathrm{E}_{1}$ and E5a signals frequencies.

$N_{G_{F F}}=\alpha_{G} \lambda_{1} N_{1}-\beta_{G} \lambda_{2} N_{2}$

$N_{E_{I F}}=\alpha_{E} \lambda_{E 1} N_{E 1}-\beta_{E} \lambda_{E 5 a} N_{E 5 a}$

where $\lambda_{1}$ and $\lambda_{2}$ are the GPS L1 and L2 signals wavelengths in meters; $\lambda_{E 1}$ and $\lambda_{E 5 a}$ are the Galileo E1 and E5a signals wavelengths in meters; $N_{1}, N_{2}$ are the integer ambiguity parameters of GPS signals L1 and L2, respectively; $N_{E I}, N_{E 5 a}$ are the integer ambiguity parameters of Galileo signals E1 and E5a, respectively.

As indicated earlier, precise orbit and satellite clock corrections from the IGS-MGEX network are used to correct both of the GPS and Galileo measurements. It should be pointed out that such products are presently referenced to the GPS time frame (Montenbruck et al., 2014b). As well, the IGS-MGEX precise GPS satellite clock corrections include the effect of the ionosphere-free linear combination of the satellite hardware delays of L1/L2 $\mathrm{P}(\mathrm{Y})$ code, while the Galileo counterpart include the effect of the ionosphere-free linear combination of the satellite hardware delays of the Galileo E1/E5a pilot code (Montenbruck et al., 2014b). Applying the precise clock corrections to Equations 5.1-5.4, we obtain: 


$$
\begin{aligned}
& P_{G_{I F}}=\rho_{G}+c\left[d t_{r G}-d t_{p r e c}^{s}\right]+c\left[\alpha d_{P 1}-\beta d_{P 2}\right]_{r}+T_{G}+\varepsilon_{P G_{I F}} \\
& P_{E_{I F}}=\rho_{E}+c\left[d t_{r G}-d t_{p r e c}^{s}\right]+c\left[\alpha d_{E 1}-\beta d_{E 5 a}\right]_{r}+T_{E}+\varepsilon_{E_{I F}} \\
& \Phi_{G_{I F}}=\rho_{G}+c d t_{r G}-c\left[d t_{p e c}^{s}+\left[\alpha d_{P 1}-\beta d_{P 2}\right]^{5}\right]+c\left[\alpha \delta_{L I}-\beta \delta_{L 2}\right]_{r}-c\left[\alpha \delta_{L I}-\beta \delta_{I 2}\right]^{s}+T_{G}+N_{G_{F F}}+\phi_{r_{G_{F F}}}+\phi_{G_{G F}}+\varepsilon_{\Phi G_{I F}} \\
& \Phi_{E_{I F}}=\rho_{E}+c a d t_{r G}-c\left[d t_{p x c}^{s}+\left[\alpha d_{E l}-\beta d_{E \Sigma a}\right]^{s}\right]+c\left[\alpha \delta_{E I}-\beta \delta_{E \Sigma a}\right]_{r}-c\left[\alpha \delta_{E I}-\beta \delta_{E \Sigma a}\right]^{s}+T_{E}+N_{E_{F F}}+\phi_{10_{E F}}+\phi_{E F F}+\varepsilon_{E E_{F F}}
\end{aligned}
$$

For simplicity, the receiver and satellite hardware delays will take the following forms:

$$
\begin{array}{ll}
b_{r_{P}}=c\left[\alpha d_{P 1}-\beta d_{P 2}\right]_{r} & b_{P}^{s}=c\left[\alpha d_{P 1}-\beta d_{P 2}\right]^{s} \\
b_{r_{E}}=c\left[\alpha d_{E 1}-\beta d_{E 5 a}\right]_{r} & b_{E}^{s}=c\left[\alpha d_{E 1}-\beta d_{E 5 a}\right]^{s} \\
b_{r_{\Phi}}=c\left[\alpha \delta_{L 1}-\beta \delta_{L 2}\right]_{r}+\phi_{r 0_{G_{I F}}} & b_{\Phi}^{s}=c\left[\alpha \delta_{L 1}-\beta \delta_{L 2}\right]^{s}+\phi_{0_{G_{I F}}}^{s} \\
b_{r_{E D}}=c\left[\alpha \delta_{E 1}-\beta \delta_{E 5 a}\right]_{r}+\phi_{r_{O_{F F}}} & b_{E \Phi}^{s}=c\left[\alpha \delta_{E 1}-\beta \delta_{E 5 a}\right]^{s}+\phi_{0_{E F F}}^{s}
\end{array}
$$

In the traditional GPS/Galileo un-differenced PPP model, the GPS receiver clock error is lumped to the GPS receiver differential code biases. In order to maintain consistency in the estimation of a common receiver clock offset, this convention is used when combining the ionosphere-free linear combination of GPS L1/L2 and Galileo E1/E5a observations. This, however, introduces an additional bias in the Galileo ionosphere-free PPP mathematical model, which represents the difference in the receiver differential code biases of both systems. Such an additional bias is commonly known as the inter-system bias (ISB). With the above consideration, the GPS/Galileo ionosphere-free linear combinations of the pseudorange and carrier-phase observations can be written as:

$$
\begin{aligned}
& P_{G_{I F}}=\rho_{G}+\tilde{d} t_{r G}-d t_{\text {prec }}^{s}+T_{G}+\varepsilon_{P G_{I F}} \\
& P_{E_{I F}}=\rho_{E}+\tilde{d} t_{r G}-d t_{\text {prec }}^{s}+I S B+T_{E}+\varepsilon_{E_{I F}} \\
& \Phi_{G_{I F}}=\rho_{G}+\tilde{d} t_{r G}-d t_{\text {prec }}^{s}+T_{G}+\tilde{N}_{G_{I F}}+\varepsilon_{\Phi G_{I F}} \\
& \Phi_{E_{I F}}=\rho_{E}+\tilde{d} t_{r G}-d t_{\text {prec }}^{s}+T_{E}+\tilde{N}_{E_{I F}}+I S B+\varepsilon_{\Phi E_{I F}}
\end{aligned}
$$


where $\tilde{d} t_{r G}$ represents the sum of the receiver clock error and receiver hardware delay $\tilde{d} t_{r G}=c d t_{r G}+b_{r_{P}} ; d t_{p r e c}^{s}$ is the precise satellite clock correction; ISB is the inter system bias, $I S B=b_{r_{E}}-b_{r_{P}} ; \tilde{N}_{G_{I F}}$ and $\tilde{N}_{E_{I F}}$ are given by:

$\tilde{N}_{G_{I F}}=N_{G_{I F}}+b_{r_{\Phi}}+b_{r_{P}}-b_{\Phi}^{s}-b_{P}^{s}$

$\tilde{N}_{E_{I F}}=N_{E_{I F}}+b_{r_{E \Phi}}+b_{r_{P}}-b_{E \Phi}^{s}-b_{E}^{s}$

When using the traditional un-differenced GPS/Galileo PPP model, the ambiguity parameters lose its integer nature as they are contaminated by the receiver and satellite hardware delays.

\subsubsection{DECOUPLED CLOCK MODEL}

The decoupled clock model assigns two different receiver and satellite clocks for the pseudorange and carrier-phase measurements (Collins et al., 2008). Applying the concept of the decoupled clock on the combined GPS and Galileo measurements and using Equations 5.1-5.4, we obtain:

$$
\begin{aligned}
& P_{G_{I F}}=\rho_{G}+c\left[d t_{r G}-d t_{G}^{S}\right]+c\left[\alpha d_{P 1}-\beta d_{P 2}\right]_{r}+c\left[\alpha d_{P 1}-\beta d_{P 2}\right]^{s}+T_{G}+\varepsilon_{P G_{I F}} \\
& P_{E_{I F}}=\rho_{E}+c\left[d t_{r E}-G G T O-d t_{E}^{S}\right]+c\left[\alpha d_{E 1}-\beta d_{E 5 a}\right]_{r}+c\left[\alpha d_{E 1}-\beta d_{E 5 a 2}\right]^{s}+T_{E}+\varepsilon_{E_{I F}} \\
& \Phi_{G_{I F}}=\rho_{G}+c\left[d t_{r G \Phi}-d t_{G \Phi}^{S}\right]+c\left[\alpha \delta_{L 1}-\beta \delta_{L 2}\right]_{r}+c\left[\alpha \delta_{L 1}-\beta \delta_{L 2}\right]^{s}+T_{G}+N_{G_{I F}}+\phi_{r 0_{G F}}+\phi_{G_{I F}}^{s}+\varepsilon_{\Phi G_{I F}} \\
& \Phi_{E_{F F}}=\rho_{E}+c\left[d t_{r E \Phi}-G G T O-d t_{E \Phi}^{S}\right]+c\left[\alpha \delta_{E l}-\beta \delta_{E S a}\right]_{r}+c\left[\alpha \delta_{E l}-\beta \delta_{E S a}\right]^{s}+T_{E}+N_{E_{F F}}+\phi_{r_{E_{F F}}}+\phi_{E_{E F}}+\varepsilon_{\Phi E_{F F}}
\end{aligned}
$$

To simplify Equations 5.17-5.20, the receiver and satellite clock errors can be written as:

$$
\begin{array}{ll}
d \tilde{t}_{r_{G}}=c d t_{r_{G}}+b_{r_{P}} & d \tilde{t}_{G}^{s}=c d t_{G}^{s}+b_{P}^{s} \\
d \tilde{t}_{r E}=c d t_{r E}+b_{r_{E}} & d \tilde{t}_{E}^{s}=c d t_{E}^{s}+b_{E}^{s} \\
d \tilde{t}_{r \Phi \Phi}=c d t_{r \Phi \Phi}+b_{r \Phi} & d \tilde{t}_{G \Phi}^{s}=c d t_{G \Phi}^{s}+b_{\Phi}^{S} \\
d \tilde{t}_{r E \Phi}=c d t_{r E \Phi}+b_{r_{E \Phi}} & d \tilde{t}_{E \Phi}^{s}=c d t_{\Phi}^{s}+b_{E \Phi}^{s}
\end{array}
$$


where $d \tilde{t}_{G}^{s}, d \tilde{t}_{E}^{s}, d \tilde{t}_{G \Phi}^{s}$, and $d \tilde{t}_{E \Phi}^{s}$ are the decoupled satellite clock errors for the pseudorange and carrier phase measurements of both GPS and Galileo systems, respectively. $d \tilde{t}_{r_{G}}, d \tilde{t}_{r_{E}}, d \tilde{t}_{r G \Phi}$, and $d \tilde{t}_{r E \Phi}$ are the receiver clock errors for the pseudorange and carrier phase measurements of both GPS and Galileo systems, respectively.

In the decoupled clock PPP model, the initial phase bias is lumped to the receiver hardware delays. As such, Equations 5.17-5.20 can be re-written as follows:

$$
\begin{aligned}
& P_{G_{I F}}=\rho_{G}+d \tilde{t}_{r G}-d \tilde{t}_{G}^{S}+T_{G}+\varepsilon_{P G_{I F}} \\
& P_{E_{I F}}=\rho_{E}+d \tilde{t}_{r E}-d \tilde{t}_{E}^{S}+T_{E}+\varepsilon_{E_{I F}} \\
& \Phi_{G_{I F}}=\rho_{G}+d \tilde{t}_{r G \Phi}-d \tilde{t}_{G \Phi}^{S}+T_{G}+N_{G_{I F}}+\varepsilon_{\Phi G_{I F}} \\
& \Phi_{E_{I F}}=\rho_{E}+d \tilde{t}_{r E \Phi}-d \tilde{t}_{E \Phi}^{S}+T_{E}+N_{E_{I F}}+\varepsilon_{\Phi E_{F F}}
\end{aligned}
$$

As shown in Equations 5.21-5.24, the assumption of having a separate receiver clock error for the pseudorange and the carrier phase observables is more complex in the case of GPS/Galileo PPP model. As all the observables are collected through a single receiver, which uses one time scale, it is uncommon to have a receiver clock error for each constellation and for each observable. As such, only the GPS receiver clock error for both of the pseudorange and carrier phase measurements is considered, assuming that the receiver uses the GPS time as a reference. Therefore, an inter-system bias term appears in the Galileo pseudorange and carrier phase equations to represent the difference between the GPS and Galileo receiver hardware delays. This leads to:

$$
\begin{aligned}
& P_{G_{I F}}=\rho_{G}+d \tilde{t}_{r G}-d \tilde{t}_{G}^{S}+T_{G}+\varepsilon_{P G_{I F}} \\
& P_{E_{I F}}=\rho_{E}+d \tilde{t}_{r G}-d \tilde{t}_{E}^{S}+I S B_{P}+T_{E}+\varepsilon_{E_{I F}} \\
& \Phi_{G_{I F}}=\rho_{G}+d \tilde{t}_{r G \Phi}-d \tilde{t}_{G \Phi}^{S}+T_{G}+N_{G_{I F}}+\varepsilon_{\Phi G_{I F}} \\
& \Phi_{E_{I F}}=\rho_{E}+d \tilde{t}_{r G \Phi}-d \tilde{t}_{E \Phi}^{S}+I S B_{C}+T_{E}+N_{E_{I F}}+\varepsilon_{\Phi E_{I F}}
\end{aligned}
$$


where the $I S B_{P}$ and $I S B_{C}$ are the pseudorange and carrier-phase inter-system biases, respectively, which are given by: $I S B_{P}=b_{r_{E \Phi}}-b_{r_{\Phi}}$ and $I S B_{C}=b_{r_{E}}-b_{r_{P}}$.

Equations 5.25-5.28 can be re-arranged by considering the satellite clock corrections and the dry tropospheric correction as follows:

$$
\begin{aligned}
& \rho_{G}+d \tilde{t}_{r G}+m_{f} z p d_{w}+\varepsilon_{P G_{I F}}-\tilde{P}_{G_{I F C}}=0 \\
& \rho_{E}+d \tilde{t}_{r G}+m_{f} z p d_{w}+I S B_{P}+\varepsilon_{E_{I F}}-\tilde{P}_{E_{I F D C}}=0 \\
& \rho_{G}+d \tilde{t}_{r G \Phi}+m_{f} z p d_{w}+N_{G_{I F}}+\varepsilon_{\Phi G_{I F}}-\tilde{\Phi}_{G_{I F_{D C}}}=0 \\
& \rho_{E}+d \tilde{t}_{r G \Phi}+m_{f} z p d_{w}+N_{E_{I F}}+I S B_{C}+\varepsilon_{\Phi E_{I F}}+\tilde{\Phi}_{E_{I F D C}}=0
\end{aligned}
$$

where, $\tilde{P}_{G_{I F C}}, \tilde{P}_{E_{I_{F C}}}, \tilde{\Phi}_{G_{I_{F} C}}$ and $\tilde{\Phi}_{E_{F_{F C}}}$ are the ionosphere-free linear combinations of the pseudorange and carrier-phase observables after applying the above corrections; $z p d_{w}$ is the wet component of the tropospheric zenith path delay; $m_{f}$ troposphere mapping functions; The ambiguity parameters of the decoupled clock PPP model are given by:

$$
\begin{aligned}
& N_{G_{I F}}=\lambda_{G_{I F}}\left[f_{1} N_{1}-f_{2} N_{2}\right] \\
& N_{E_{I F}}=\lambda_{E_{I F}}\left[f_{E 1} N_{1}-f_{E 5 a} N_{2}\right]
\end{aligned}
$$

Figures 5.3-5.5 show the decoupled precise satellite clock corrections for the pseudorange and carrier-phase observations for different days, namely August 26 and 27 of 2012, and April 5, 2013. As indicated earlier, the difference between the decoupled satellite clock corrections is the satellite hardware delay for pseudorange and carrier phase observations as shown in Figures 5.3-5.5. Only the GPS decoupled clock products are presented in this paper because of the unavailability of the Galileo decoupled clock products at present.

As shown in Figures 5.3 and 5.4, the difference between the IGS (pseudorange) and decoupled (carrier phase) clock corrections is essentially constant. However, in Figure 5.5, which the data used represent around 7 months after the data used for Figures 5.3 and 5.4, the difference 
between the IGS and decoupled clock corrections is different than the ones in Figures 5.3 and 5.4 as shown in Table 5.1.
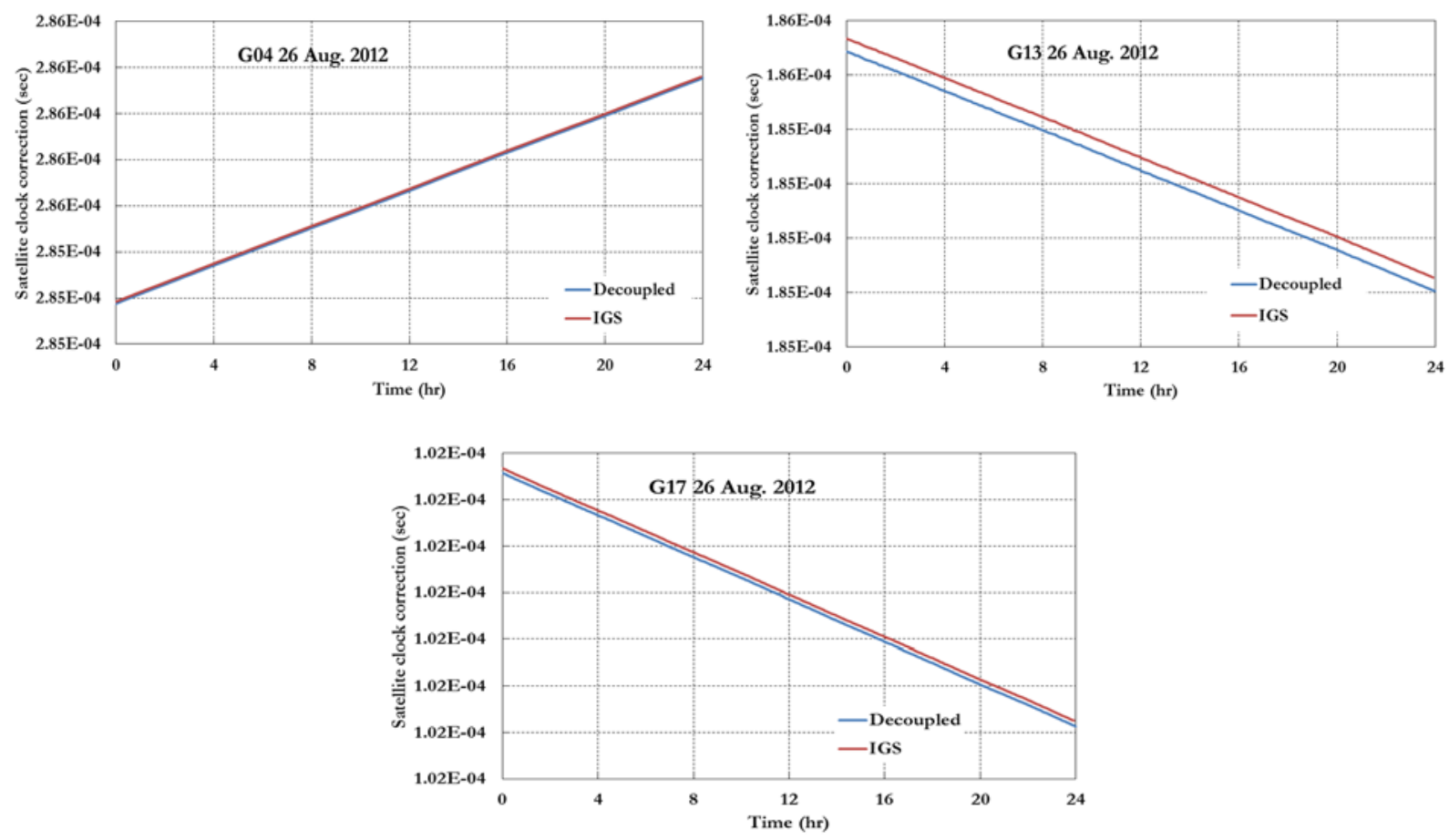

Figure 5. 3 IGS and decoupled clock corrections 26 August 2012
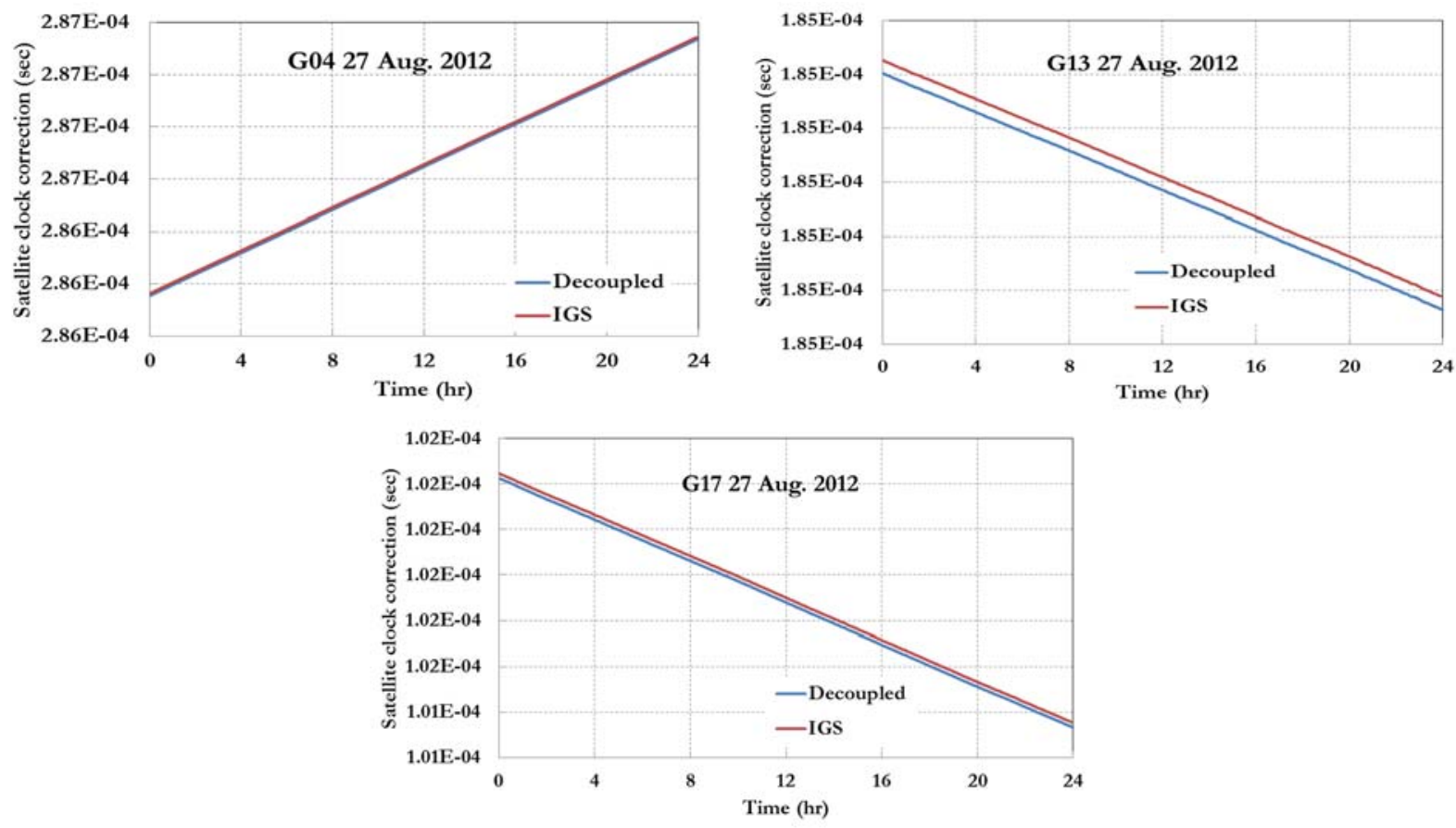
Figure 5. 4 IGS and decoupled clock corrections 27 August 2012
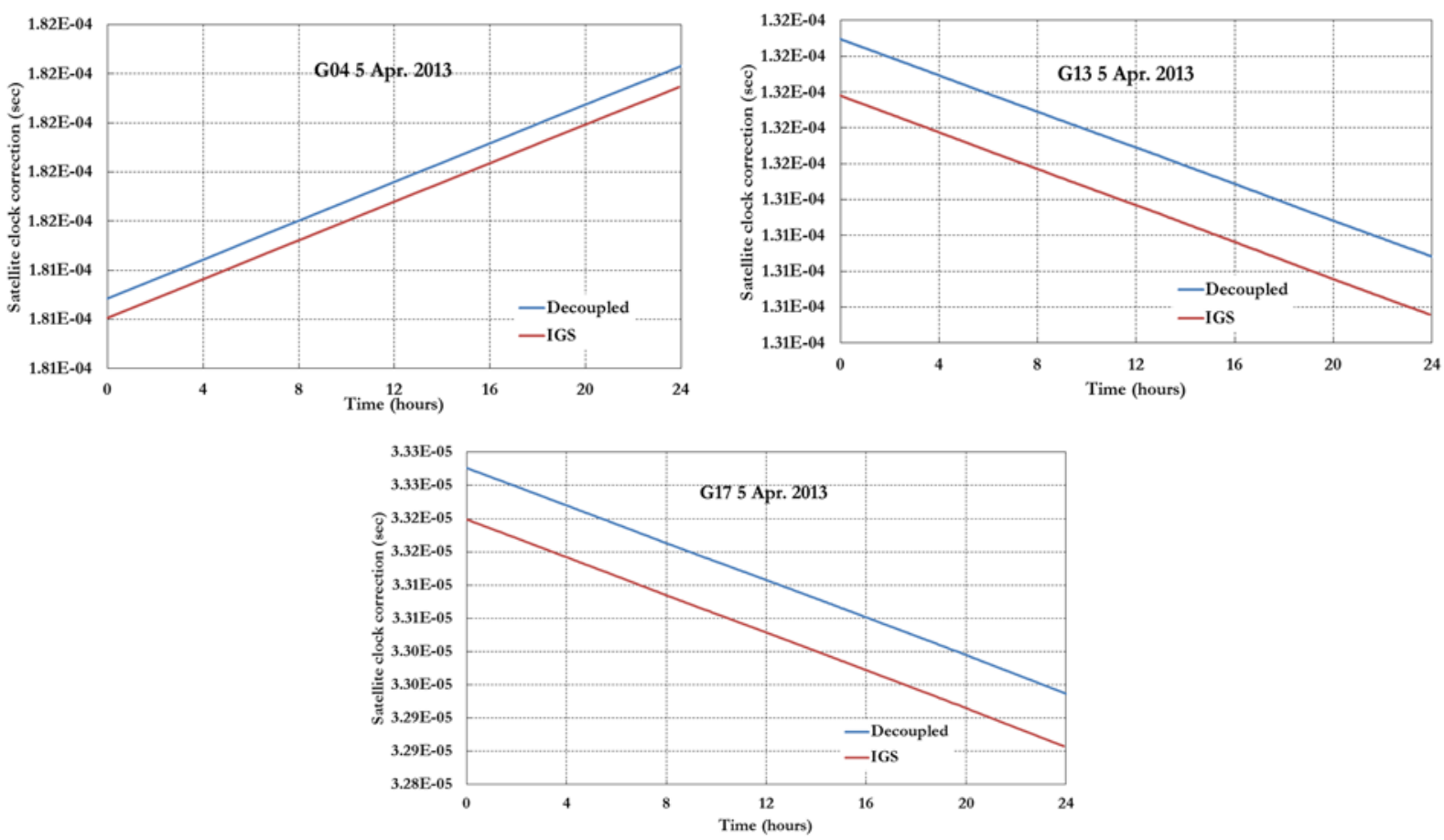

Figure 5. 5 IGS and decoupled clock corrections 5 April 2013

As shown in Table 1, the difference between the IGS and decoupled precise clock correction can be assumed to be constant for the short period of time extent to days; however, for long term cases the difference will not be constant. As a result, it can be concluded that either satellite clock corrections or un-calibrated satellite hardware delays drift over time or even both change over time.

Table 5. 1 Satellite clock correction difference between decoupled and IGS products

\begin{tabular}{cccc}
\hline \multirow{2}{*}{ Date } & \multicolumn{3}{c}{ Satellite Clock Correction Difference (Decoupled-IGS) (s) } \\
\cline { 2 - 4 } & $\mathbf{G 0 4}$ & $\mathbf{G 1 3}$ & $\mathbf{G 1 7}$ \\
\hline 26 August 2012 & $-6.91 \mathrm{E}-09$ & $-1.20 \mathrm{E}-08$ & $-5.60 \mathrm{E}-09$ \\
27 August 2012 & $-6.91 \mathrm{E}-09$ & $-1.20 \mathrm{E}-08$ & $-5.60 \mathrm{E}-09$ \\
05 April 2013 & $8.19 \mathrm{E}-08$ & $7.99 \mathrm{E}-08$ & $7.83 \mathrm{E}-08$ \\
\hline
\end{tabular}

As shown in Table 1, the difference between the IGS and decoupled precise clock correction can be assumed to be constant for the short period of time extent to days; however, for long term cases the difference will not be constant. As a result, it can be concluded that either satellite 
clock corrections or un-calibrated satellite hardware delays drift over time or even both change over time.

\subsubsection{SEMI-DECOUPLED CLOCK GPS/GALILEO PPP MODEL}

In this model, the IGS-MGEX network precise clock corrections and the daily DCB for both GPS/Galileo satellites are used (IGS, 2015; Montenbruck, et al., 2014a). Considering the carrierphase DCB, Equations 5.29-5.31 can be rewritten as:

$$
\begin{aligned}
& \rho_{G}+d \tilde{t}_{r G}+m_{f} z p d_{w}+\varepsilon_{P G_{I F}}-\tilde{P}_{G_{I F}}=0 \\
& \rho_{E}+d \tilde{t}_{r G}+m_{f} z p d_{w}+I S B_{P}+\varepsilon_{E_{I F}}-\tilde{P}_{E_{I F}}=0 \\
& \rho_{G}+d \tilde{t}_{r G \Phi}+m_{f} z p d_{w}+\tilde{N}_{G_{I F}}+\varepsilon_{\Phi G_{I F}}-\tilde{\Phi}_{G_{I F}}=0 \\
& \rho_{E}+d \tilde{t}_{r G \Phi}+m_{f} z p d_{w}+\tilde{N}_{E_{I F}}+I S B_{C}+\varepsilon_{\Phi E_{I F}}+\tilde{\Phi}_{E_{I F}}=0
\end{aligned}
$$

The carrier phase satellite hardware delays will be lumped to the ambiguity parameters as shown in Equations 5.39 and 5.40.

$$
\begin{aligned}
& \tilde{N}_{G_{I F}}=\lambda_{G_{I F}}\left[f_{1} N_{1}-f_{2} N_{2}\right]+b_{\Phi}^{S} \\
& \tilde{N}_{E_{I F}}=\lambda_{E_{I F}}\left[f_{E 1} N_{1}-f_{E S a} N_{2}\right]+b_{E \Phi}^{S}
\end{aligned}
$$

\subsection{BSSD GPS/GALILEO MODELS}

\subsubsection{TRADITIONAL BSSD GPS/GALILEO PPP MODEL}

As indicated earlier, two scenarios are considered when forming the BSSD linear combination, namely a tight and a loose combination. In the first scenario, either a GPS or a Galileo satellite is selected as a reference for both GPS and Galileo observables (Afifi and El-Rabbany, 2015). Taking a GPS satellite as a reference and using Equations 5.11 to 5.14, we obtain:

$\rho_{G, G}^{i j}+m_{f}^{i j} z p d_{w}+\tilde{\varepsilon}_{P G_{I F}}^{i j}-\tilde{P}_{G_{I F}}^{i j}=0$ 
$\rho_{E, G}^{i k}+m_{f}^{i k} z p d_{w}+I S B+\tilde{\varepsilon}_{P E_{I F}}^{i k}-\tilde{P}_{E G_{I F}}^{i k}=0$

$\rho_{G, G}^{i j}+m_{f}^{i j} z p d_{w}+\tilde{N}_{G_{I F}}^{i j}+\tilde{\varepsilon}_{\Phi G_{I F}}^{i j}-\tilde{\Phi}_{G_{I F}}^{i j}=0$

$\rho_{E, G}^{i k}+m_{f}^{i k} z p d_{w}+I S B+\tilde{N}_{E G_{I F}}^{i k}+\tilde{\varepsilon}_{\Phi E_{I F}}^{i k}-\tilde{\Phi}_{E G_{I F}}^{i k}=0$

where, the superscript $i$ refers to the GPS reference satellite; the superscripts $j$ and $k$ refer the GPS and Galileo satellites respectively; $\tilde{N}_{G_{I F}}^{i j}$ and $\tilde{N}_{E G_{I F}}^{i k}$ are given by:

$$
\begin{aligned}
& \tilde{N}_{G_{\text {IF }}}^{i j}=N_{G_{\text {IF }}}^{i}-N_{G_{\text {IFF }}}^{j}+b_{G \Phi}^{i j}-b_{P}^{i j} \\
& \tilde{N}_{E G_{F F}}^{i k}=N_{G_{F F}}^{i}-N_{E_{F F}}^{k}+b_{r_{E \Phi}}-b_{r_{\Phi}}+b_{E \Phi}^{k}-b_{\Phi}^{i}+b_{P}^{i}-b_{E}^{k}
\end{aligned}
$$

As shown in Equation 5.45, the GPS ambiguity parameters include only the GPS satellite hardware delays. However, for Galileo system, the ambiguity parameters include both of the receiver and satellite hardware delays. Similarly, when a Galileo satellite is selected as a reference, using Equations 5.11 to 5.14 leads to:

$$
\begin{aligned}
& \rho_{G, E}^{l j}+m_{f}^{l j} z p d_{w}-I S B+\tilde{\varepsilon}_{P G_{I F}}^{l j}-\tilde{P}_{G E_{I F}}^{l j}=0 \\
& \rho_{E, E}^{l k}+m_{f}^{l k} z p d_{w}+\tilde{\varepsilon}_{P E_{I F}}^{l k}-\tilde{P}_{E_{I F}}^{l k}=0 \\
& \rho_{G, E}^{l j}+m_{f}^{l j} z p d_{w}-I S B+\tilde{N}_{G E_{I F}}^{l j}+\tilde{\varepsilon}_{\Phi G_{I F}}^{l j}-\tilde{\Phi}_{G E_{I F}}^{l j}=0 \\
& \rho_{E, E}^{l k}+m_{f}^{l k} z p d_{w}+\tilde{N}_{E_{I F}^{l k}}^{l k}+\tilde{\varepsilon}_{\Phi E_{I F}^{l k}}^{l k}-\tilde{\Phi}_{E_{I F}}^{l k}=0
\end{aligned}
$$

where, the superscript $l$ refers to the Galileo reference satellite, $\tilde{N}_{G E_{I F}}^{l j}$ and $\tilde{N}_{E_{I F}}^{l k}$ are the BSSD non-integer ambiguity parameters lumped to the receiver and satellite hardware delays, which are given by:

$$
\begin{aligned}
& \tilde{N}_{G E_{I F}}^{l j}=N_{E_{I F}}^{l}-N_{G_{I F}}^{j}+b_{r_{G \Phi}}-b_{r_{E \Phi}}+b_{G \Phi}^{j}-b_{E \Phi}^{l}+b_{E}^{l}-b_{P}^{j} \\
& \tilde{N}_{E_{I F}}^{l k}=N_{E_{I F}}^{l}-N_{E_{I F}}^{k}+b_{E \Phi}^{l k}-b_{E}^{l k}
\end{aligned}
$$

In the loose BSSD combination, two reference satellites are considered: a GPS reference satellite for the GPS observables and a Galileo satellite for the Galileo observables. Using Equations 5.11 to 5.14 , we obtain: 
$\rho_{G, G}^{i j}+m_{f}^{i j} z p d_{w}+\tilde{\varepsilon}_{P G_{I F}}^{i j}-\tilde{P}_{G_{I F}}^{i j}=0$

$\rho_{E, E}^{l k}+m_{f}^{l k} z p d_{w}+\widetilde{\varepsilon}_{P E_{I F}}^{l k}-\tilde{P}_{E_{I F}}^{l k}=0$

$\rho_{G, G}^{i j}+m_{f}^{i j} z p d_{w}+\tilde{N}_{G_{I F}}^{i j}+\tilde{\varepsilon}_{\Phi G_{I F}}^{i j}-\tilde{\Phi}_{G_{I F}}^{i j}=0$

$\rho_{E, E}^{l k}+m_{f}^{l k} z p d_{w}+\tilde{N}_{E_{I F}}^{l k}+\tilde{\varepsilon}_{\Phi E_{I F}}^{l k}-\tilde{\Phi}_{E_{I F}}^{l k}=0$

where, $\tilde{N}_{G_{I F}}^{i j}$ and $\tilde{N}_{E_{I F}}^{l k}$ are the BSSD non-integer ambiguity parameters lumped to the receiver and satellite hardware delays as shown in Equations 5.57 and 5.58.

$\tilde{N}_{G_{I F}}^{i j}=N_{G_{I F}}^{i}-N_{G_{I F}}^{j}+b_{G \Phi}^{i j}-b_{P}^{i j}$

$\tilde{N}_{E_{I F}}^{l k}=N_{E_{I F}}^{l}-N_{E_{I F}}^{k}+b_{E \Phi}^{l k}-b_{E}^{l k}$

In this case, all receiver hardware delays are canceled out for both systems. The major advantage of the above per-constellation system is that both of the receiver clock error and the inter-system bias are cancelled out.

\subsubsection{BSSD DECOUPLED CLOCK GPS/GALILEO PPP MODEL}

The BSSD decoupled clock model can be formed by using a GPS satellite as a reference. Using Equations 5.29 to 5.32, we obtain:

$\rho_{G, G}^{i j}+m_{f}^{i j} z p d_{w}+\tilde{\varepsilon}_{P G_{I F}}^{i j}-\tilde{P}_{G_{I F}}^{i j}=0$

$\rho_{E, G}^{i k}+m_{f}^{i k} z p d_{w}+I S B_{P}+\widetilde{\varepsilon}_{P E_{I F}}^{i k}-\tilde{P}_{E G_{I F}}^{i k}=0$

$\rho_{G, G}^{i j}+m_{f}^{i j} z p d_{w}+\tilde{N}_{G_{I F}}^{i j}+\tilde{\varepsilon}_{\Phi G_{I F}}^{i j}-\tilde{\Phi}_{G_{I F}}^{i j}=0$

$\rho_{E, G}^{i k}+m_{f}^{i k} z p d_{w}+I S B_{C}+\tilde{N}_{E G_{I F}}^{i k}+\tilde{\varepsilon}_{\Phi E_{I F}}^{i k}-\tilde{\Phi}_{E G_{I F}}^{i k}=0$

where, $\tilde{N}_{G_{I F}}^{i j}$ and $\tilde{N}_{E_{I F}}^{i j}$ are given respectively by:

$\tilde{N}_{G_{I F}}^{j j}=N_{G_{I F}}^{\dot{j}}-N_{G_{I F}}^{j}$ 
$\tilde{N}_{E G_{I F}}^{k}=N_{G_{I F}}^{\dot{j}}-N_{E_{I F}}^{k}$

When a Galileo satellite is selected as a reference, the BSSD equations take the form:

$\rho_{G, E}^{l j}+m_{f}^{l j} z p d_{w}-I S B_{P}+\tilde{\varepsilon}_{P G_{I F}}^{l j}-\tilde{P}_{G E_{I F}}^{l j}=0$

$\rho_{E, E}^{l k}+m_{f}^{l k} z p d_{w}+\tilde{\varepsilon}_{P E_{I F}}^{l k}-\tilde{P}_{E_{I F}}^{l k}=0$

$\rho_{G, E}^{l j}+m_{f}^{l j} z p d_{w}-I S B_{C}+\tilde{N}_{G E_{I F}}^{l j}+\tilde{\varepsilon}_{\Phi G_{I F}}^{l j}-\tilde{\Phi}_{G E_{I F}}^{l j}=0$

$\rho_{E, E}^{l k}+m_{f}^{l k} z p d_{w}+\tilde{N}_{E_{I F}}^{l k}+\tilde{\varepsilon}_{\Phi E_{I F}}^{l k}-\tilde{\Phi}_{E_{I F}}^{l k}=0$

where, $\tilde{N}_{G_{I F}}^{i j}$ and $\tilde{N}_{E_{I F}}^{i j}$ are given by Equations 5.69 and 5.70, respectively.

$$
\begin{aligned}
& \tilde{N}_{G E_{I F}}^{j j}=N_{E_{I F}}^{t}-N_{G_{I F}}^{j} \\
& \tilde{N}_{E_{I F}}^{k k}=N_{E_{I F}}-N_{E_{I F}}^{k}
\end{aligned}
$$

In the per-constellation BSSD model, two reference satellites are selected are references, a GPS and a Galileo. Using Equations 5.29-5.32, we obtain:

$$
\begin{aligned}
& \rho_{G, G}^{i j}+m_{f}^{i j} z p d_{w}+\tilde{\varepsilon}_{P G_{I F}}^{i j}-\tilde{P}_{G_{I F}}^{i j}=0 \\
& \rho_{E, E}^{l k}+m_{f}^{l k} z p d_{w}+\tilde{\varepsilon}_{P E_{I F}}^{l k}-\tilde{P}_{E_{I F}}^{l k}=0 \\
& \rho_{G, G}^{i j}+m_{f}^{i j} z p d_{w}+\tilde{N}_{G_{I F}}^{i j}+\tilde{\varepsilon}_{\Phi G_{I F}}^{i j}-\tilde{\Phi}_{G_{I F}}^{i j}=0 \\
& \rho_{E, E}^{l k}+m_{f}^{l k} z p d_{w}+\tilde{N}_{E_{I F}}^{l k}+\tilde{\varepsilon}_{\Phi E_{I F} l k}^{l k}-\tilde{\Phi}_{E_{I F}}^{l k}=0
\end{aligned}
$$

As can be seen in Equations 5.75-5.78, the ISB terms are cancelled. The differenced ambiguity parameters $\tilde{N}_{G_{I F}}^{i j}$ and $\tilde{N}_{E_{I F}}^{i j}$ can still be obtained from Equations 5.75 and 5.76, respectively.

$$
\begin{aligned}
& \tilde{N}_{G_{I F}}^{j j}=N_{G_{I F}}^{j}-N_{G_{I F}}^{j} \\
& \tilde{N}_{E_{I F}}^{k}=N_{E_{F F}}^{\dagger}-N_{E_{F F}}^{k}
\end{aligned}
$$




\subsubsection{BSSD SEMI-DECOUPLED CLOCK GPS/GALILEO PPP MODEL}

The BSSD semi-decoupled clock model can be formed by using a GPS satellite as a reference. Using Equations 5.35 to 5.38, we obtain:

$\rho_{G, G}^{i j}+m_{f}^{i j} z p d_{w}+\tilde{\varepsilon}_{P G_{I F}}^{i j}-\tilde{P}_{I_{I F}}^{i j}=0$

$\rho_{E, G}^{i k}+m_{f}^{i k} z p d_{w}+I S B_{P}+\tilde{\varepsilon}_{P E_{I F}}^{i k}-\tilde{P}_{E G_{I F}}^{i k}=0$

$\rho_{G, G}^{i j}+m_{f}^{i j} z p d_{w}+\tilde{N}_{G_{I F}}^{i j}+\tilde{\varepsilon}_{\Phi G_{I F}}^{i j}-\tilde{\Phi}_{G_{I F}}^{i j}=0$

$\rho_{E, G}^{i k}+m_{f}^{i k} z p d_{w}+I S B_{C}+\tilde{N}_{E G_{I F}^{i k}}^{i k}+\tilde{\varepsilon}_{\mathscr{G} E_{I F}}^{i k}-\tilde{\Phi}_{E G_{I F}}^{i k}=0$

where, $\tilde{N}_{G_{I F}}^{i j}$ and $\tilde{N}_{E_{I F}}^{i j}$ are given respectively by:

$\tilde{N}_{G_{F F}}^{i j}=N_{G_{F F}}^{i}-N_{G_{F F}}^{j}+b_{\Phi}^{i}-b_{\Phi}^{j}$

$\tilde{N}_{E G_{F F}}^{k}=N_{E_{F F}}^{j}-N_{G_{F F}}^{k}+b_{\Phi}^{i}-b_{E \Phi}^{k}$

When a Galileo satellite is selected as a reference, the BSSD equations take the form:

$\rho_{G, E}^{l j}+m_{f}^{l j} z p d_{w}-I S B_{P}+\tilde{\varepsilon}_{P G_{I F}}^{l j}-\tilde{P}_{G E_{I F}}^{l j}=0$

$\rho_{E, E}^{l k}+m_{f}^{l k} z p d_{w}+\tilde{\varepsilon}_{P E_{I F}}^{l k}-\tilde{P}_{E_{I F}}^{l k}=0$

$\rho_{G, E}^{l j}+m_{f}^{l j} z p d_{w}-I S B_{C}+\tilde{N}_{G E_{I F}}^{j}+\tilde{\varepsilon}_{\Phi G_{I F}}^{l j}-\tilde{\Phi}_{G E_{I F}}^{l j}=0$

$\rho_{E, E}^{l k}+m_{f}^{l k} z p d_{w}+\tilde{N}_{E_{I F}}^{l k}+\tilde{\varepsilon}_{\Phi E_{I F}}^{l k}-\tilde{\Phi}_{E_{I F}}^{l k}=0$

where, $\tilde{N}_{G_{I F}}^{i j}$ and $\tilde{N}_{E_{I F}}^{i j}$ are given by Equations 87 and 88 , respectively.

$\tilde{N}_{G E_{F F}}^{j j}=N_{E_{F F}}^{t}-N_{G_{I F}}^{j}+b_{E \Phi}^{l}-b_{\Phi}^{j}$

$\tilde{N}_{E_{F}}^{k}=N_{E_{F F}}^{t}-N_{E_{F F}}^{k}+b_{E \Phi}^{b}-b_{E \Phi}^{k}$ 
In the per-constellation BSSD model, two reference satellites are selected are references, a GPS and a Galileo. Using Equations 5.35-5.38, we obtain:

$\rho_{G, G}^{i j}+m_{f}^{i j} z p d_{w}+\tilde{\varepsilon}_{P G_{I F}}^{i j}-\tilde{P}_{G_{I F}}^{i j}=0$

$\rho_{E, E}^{l k}+m_{f}^{l k} z p d_{w}+\widetilde{\varepsilon}_{P E_{I F}}^{l k}-\tilde{P}_{E_{I F}}^{l k}=0$

$\rho_{G, G}^{i j}+m_{f}^{i j} z p d_{w}+\tilde{N}_{G_{I F}}^{i j}+\tilde{\varepsilon}_{\Phi G_{I F}}^{i j}-\tilde{\Phi}_{G_{I F}}^{i j}=0$

$\rho_{E, E}^{l k}+m_{f}^{l k} z p d_{w}+\tilde{N}_{E_{I F}}^{l k}+\tilde{\varepsilon}_{\Phi E_{I F}}^{l k}-\tilde{\Phi}_{E_{I F}}^{l k}=0$

As can be seen in Equations 5.89-5.92, the ISB terms are cancelled out. The differenced ambiguity parameters $\tilde{N}_{G_{I F}}^{i j}$ and $\tilde{N}_{E_{I F}}^{i j}$ can still be obtained through Equations 5.93 and 5.94, respectively.

$$
\begin{aligned}
& \tilde{N}_{G_{I F}}^{i j}=N_{G_{I F}}^{i}-N_{G_{I F}}^{j}+b_{\Phi}^{i}-b_{\Phi}^{j} \\
& \tilde{N}_{E_{I F}}^{k}=N_{E_{I F}}^{j}-N_{E_{I F}}^{k}+b_{E \Phi}^{l}-b_{E \Phi}^{k}
\end{aligned}
$$

\subsection{LEAST SQUARES ESTIMATION TECHNIQUE}

Under the assumption that the observations are uncorrelated and the errors are normally distributed with zero mean, the covariance matrix of the un-differenced observations takes the form of a diagonal matrix. The elements along the diagonal line represent the variances of the code and carrier phase measurements. Following the common practice, the ratio between the standard deviations of the code and the carrier-phase measurements is taken as 100 . When forming BSSD, however, the differenced observations become mathematically correlated. This leads to a fully populated covariance matrix at a particular epoch.

The general linearized form for the above observation equations around the initial (approximate) vector $\boldsymbol{u}^{0}$ and observables $l$ can be written in a compact form as:

$f\left(u_{g} l\right)=A \Delta u-w-r=0$ 
where $\boldsymbol{u}$ is the vector of unknown parameters; $\boldsymbol{A}$ is the design matrix, which includes the partial derivatives of the observation equations with respect to the unknown parameters $\boldsymbol{u} ; \boldsymbol{\Delta} \boldsymbol{u}$ is the unknown vector of corrections to the approximate parameters $\boldsymbol{u}^{0}$, i.e., $\boldsymbol{u}=\boldsymbol{u}^{0}+\boldsymbol{\Delta} \boldsymbol{u} ; \boldsymbol{w}$ is the misclosure vector and $\boldsymbol{r}$ is the vector of residuals. The sequential least-squares solution for the unknown parameters $\boldsymbol{\Delta} \boldsymbol{u}_{i}$ at an epoch $i$ can be obtained from (Vanicek and Krakiwsky, 1986):

$$
\begin{aligned}
& \Delta \boldsymbol{u}_{i}=\Delta \boldsymbol{u}_{i-1}+\boldsymbol{M}_{i-1}^{-1} \boldsymbol{A}_{i}^{T}\left(\boldsymbol{C}_{l_{i}}+\boldsymbol{A}_{i} \boldsymbol{M}_{i-1}^{-1} \boldsymbol{A}_{i}^{T}\right)^{-1}\left[\boldsymbol{w}_{i}-\boldsymbol{A}_{i} \Delta \boldsymbol{u}_{i-1}\right] \\
& \boldsymbol{M}_{i}^{-1}=\boldsymbol{M}_{i-1}^{-1}-\boldsymbol{M}_{i-1}^{-1} \boldsymbol{A}_{i}^{T}\left(\boldsymbol{C}_{l_{i}}+\boldsymbol{A}_{i} \boldsymbol{M}_{i-1}^{-1} \boldsymbol{A}^{T}\right)^{-1} \boldsymbol{A}_{i} \boldsymbol{M}_{i-1}^{-1} \\
& \boldsymbol{C}_{\Delta u_{i}}=\boldsymbol{M}_{i}^{-1}=\boldsymbol{M}_{i-1}^{-1}-\boldsymbol{M}_{i-1}^{-1} \boldsymbol{A}_{i}^{T}\left(\boldsymbol{C}_{l_{i}}+\boldsymbol{A}_{i} \boldsymbol{M}_{i-1}^{-1} \boldsymbol{A}^{T}\right)^{-1} \boldsymbol{A}_{i} \boldsymbol{M}_{i-1}^{-1}
\end{aligned}
$$

where $\boldsymbol{\Delta} \boldsymbol{u}_{i-1}$ is the least-squares solution for the estimated parameters at epoch $i-1 ; \boldsymbol{M}$ is the matrix of the normal equations; $\boldsymbol{C}_{l}$ and $\boldsymbol{C}_{\Delta u}$ are the covariance matrices of the observations and unknown parameters, respectively. It should be pointed out that the usual batch least-squares adjustment should be used in the first epoch, i.e., for $i=1$. The batch solution for the estimated parameters and the inverse of the normal equation matrix are given, respectively, by (Vanicek and Krakiwsky, 1986):

$$
\begin{aligned}
& \Delta \boldsymbol{u}_{l}=\left[\boldsymbol{C}_{x^{0}}^{-l}+\boldsymbol{A}_{l}^{T} \boldsymbol{C}_{l_{1}}^{-l} \boldsymbol{A}_{l}\right]^{-1} \boldsymbol{A}_{l}^{T} \boldsymbol{C}_{l_{1}}^{-l} \boldsymbol{w}_{l} \\
& \boldsymbol{M}_{1}^{-l}=\left[\boldsymbol{C}_{x^{0}}^{-l}+\boldsymbol{A}_{l}^{T} \boldsymbol{C}_{l_{1}}^{-l} \boldsymbol{A}_{l}\right]^{-1}
\end{aligned}
$$

where $\boldsymbol{C}_{x}{ }^{0}$ is a priori covariance matrix for the approximate values of the unknown parameters.

In case of the traditional GPS/Galileo PPP model, the design matrix $A$ and the vector of corrections to the unknown parameters $\Delta \mathrm{u}$ take the following forms: 


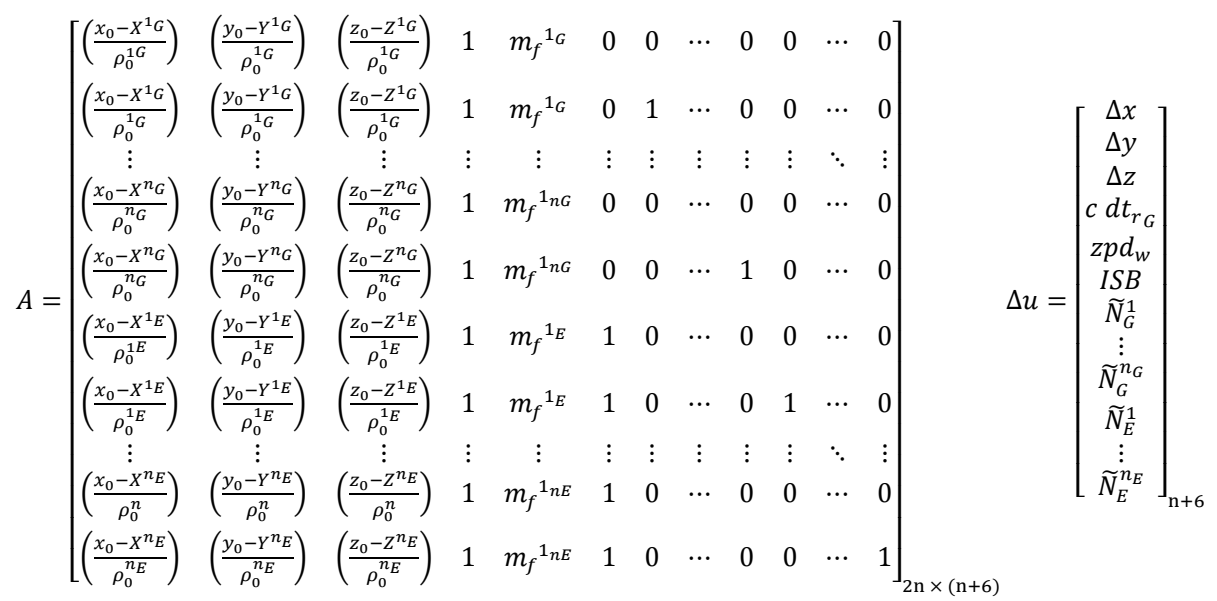

where $n_{G}$ refers to the number of visible GPS satellites; $n_{E}$ refers to the number of visible Galileo satellites; $n=n_{G}+n_{E}$ is the total number of the observed satellites for both GPS/Galileo systems; $x_{0}, y_{0}$ and $z_{0}$ are the approximate receiver coordinates; $X^{j_{G}}, Y^{j_{G}}, Z^{j_{G}}, j=1,2, \ldots, n_{G}$ are the known GPS satellite coordinates; $X^{k_{E}}, Y^{k_{E}}, Z^{k_{E}}, k=1,2, \ldots, n_{E}$ are the known Galileo satellite coordinates; $\rho_{0}$ is the approximate receiver-satellite range. The unknown parameters in the above system are the corrections to the receiver coordinates, $\Delta x, \Delta y$, and $\Delta z$, the wet component of the tropospheric zenith path delay $z p d_{w}$, the inter-system bias $I S B$, and the non-integer ambiguity parameters $\widetilde{N}$.

For the decoupled clock model, the design matrix $A$ and the vector of corrections to the unknown parameters $\Delta \mathrm{u}$ take the following forms:

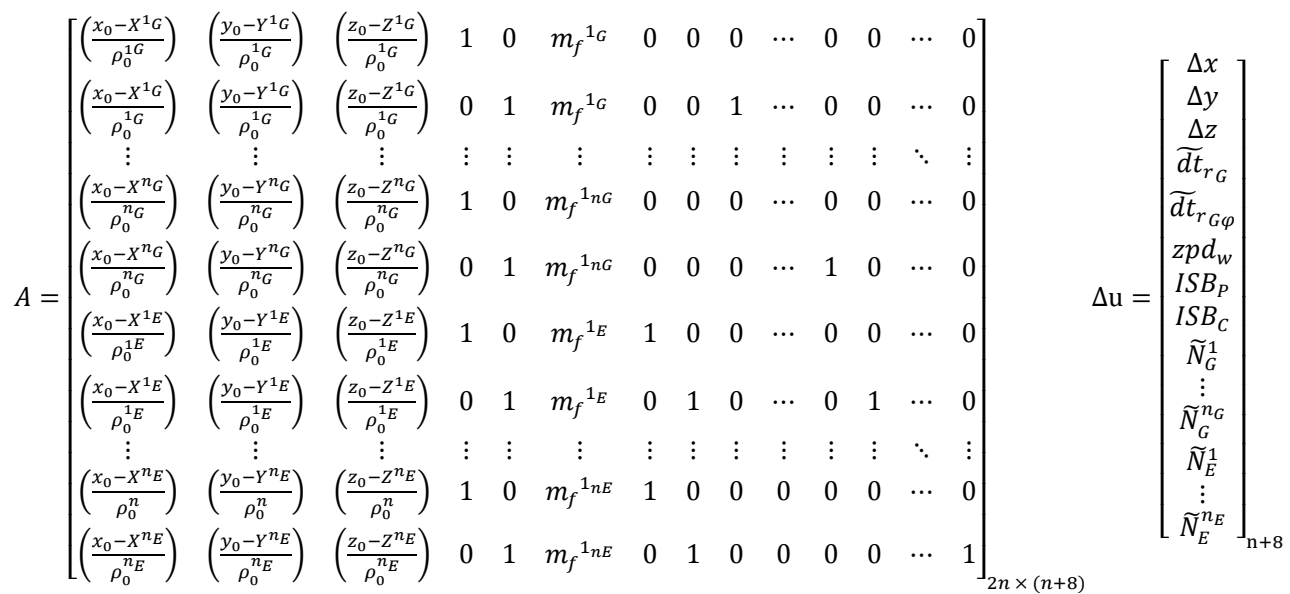

where $d t_{r_{G}}$ and $d t_{r_{G \Phi}}$ are the pseudorange and carrier phase receiver clock errors, respectively; $I S B_{P}$ and $I S B_{C}$ are the pseudorange and carrier phase inter-system bias, respectively. 
For the un-differenced semi-decoupled clock GPS/Galileo PPP model, the design matrix $\boldsymbol{A}$ and the vector of corrections to the unknown parameters $\Delta x$ take the same form as the decoupled clock GPS/Galileo model given in Equation (5.102). When a GPS satellite is selected as a reference to form the BSSD for GPS/Galileo observations, the design matrix $\boldsymbol{A}$ and the vector of corrections $\boldsymbol{\Delta} \boldsymbol{u}$ take the form:

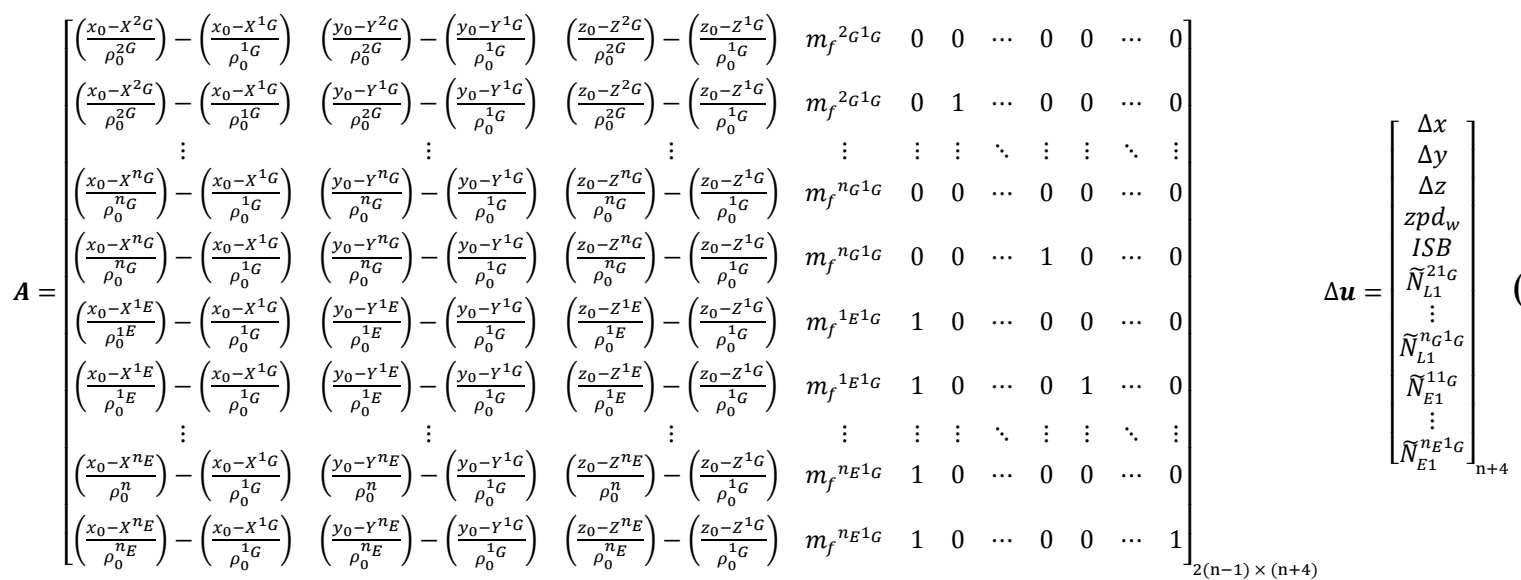

where " $l_{G}$ " refers to the GPS reference satellite.

By analogy, the use of a Galileo satellite as a reference to form the BSSD for both of the GPS and Galileo observations leads to:

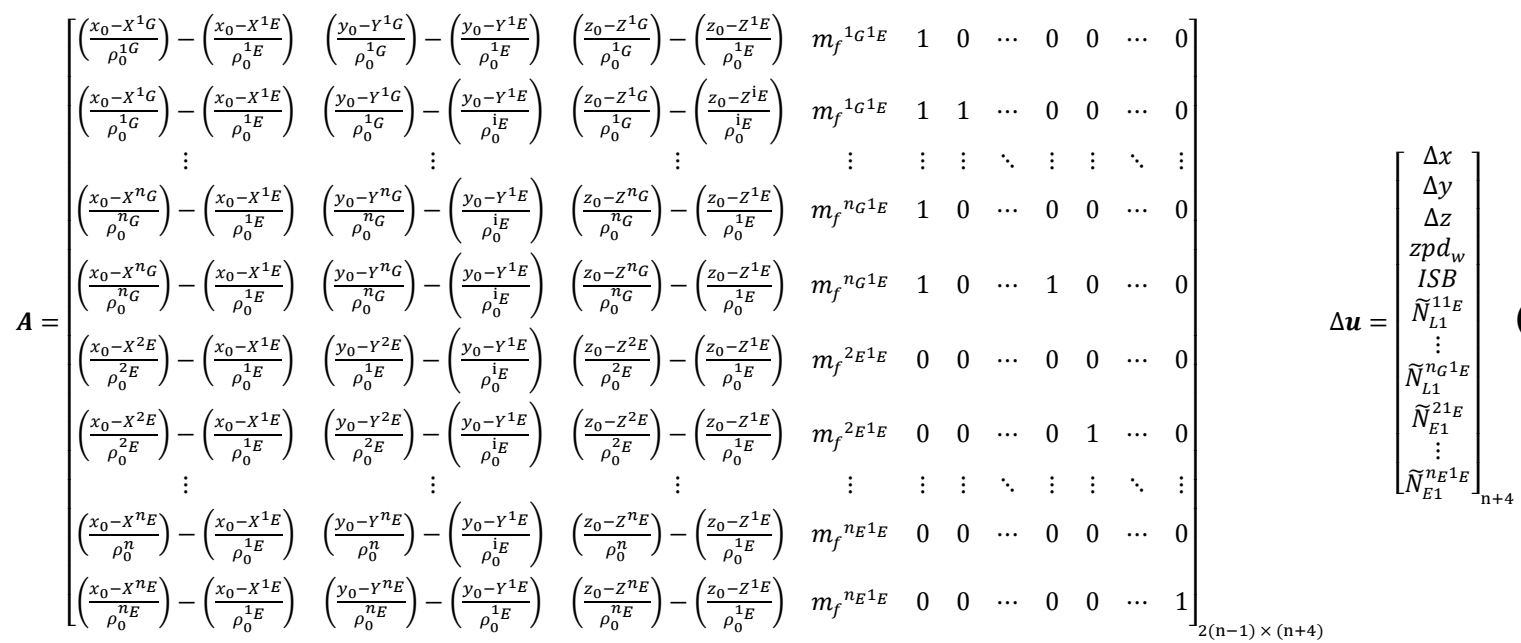

where $l_{E}$ refers to the Galileo reference satellite. 
When two reference satellites are selected to form the BSSD, i.e., per-constellation BSSD, the design matrix $\boldsymbol{A}$ and the vector of corrections $\boldsymbol{\Delta} \boldsymbol{u}$ take the form:

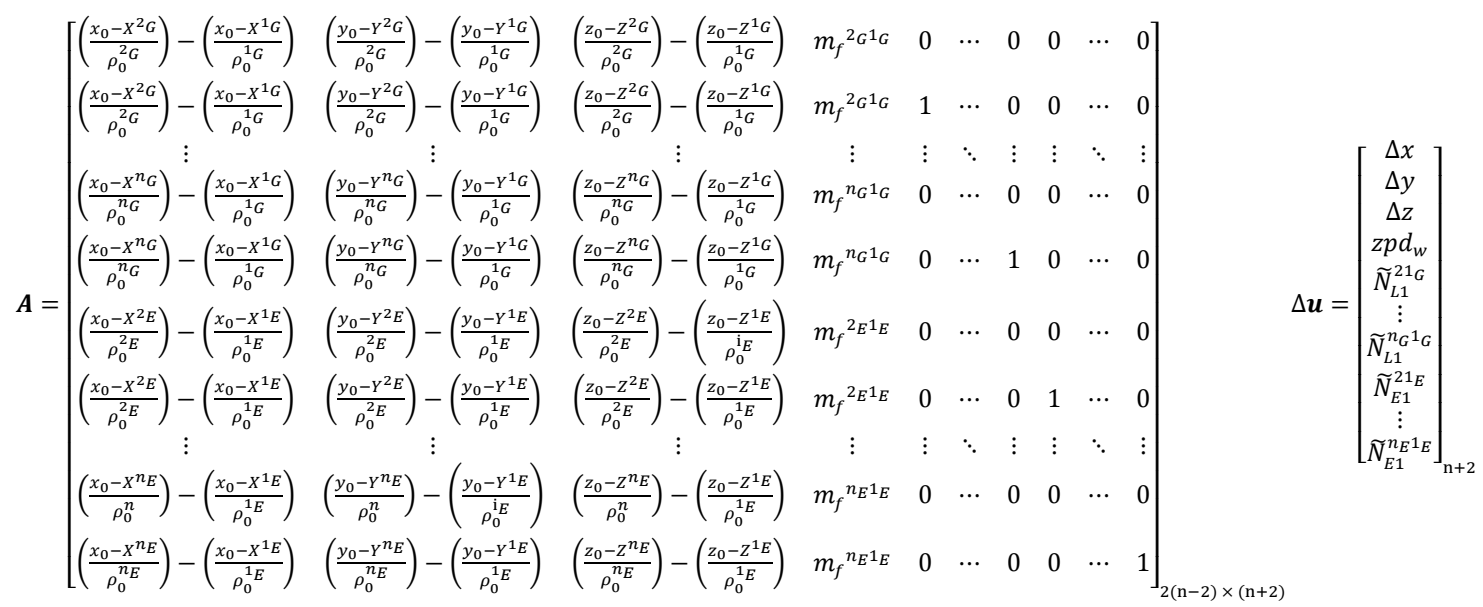

The major advantage of the above per-constellation (or loose combination) system is that the modified receiver clock error and the inter-system bias are cancelled out. Similarly, the design matrix $\boldsymbol{A}$ and the vector of corrections $\boldsymbol{\Delta} \boldsymbol{u}$ for the BSSD decoupled clock model, with a GPS satellite selected as a reference, take the form:

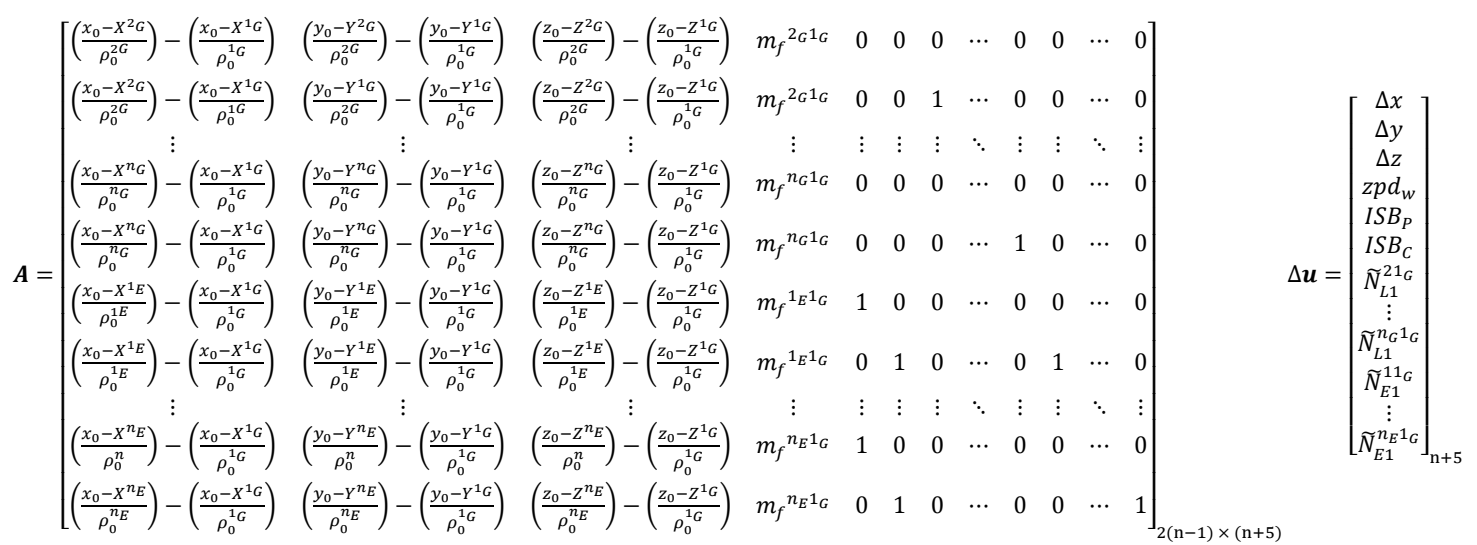

where " $l_{G}$ " refers to the GPS reference satellite.

If, however, a Galileo satellite is selected as a reference, the design matrix $\boldsymbol{A}$ and the vector of corrections $\boldsymbol{\Delta} \boldsymbol{u}$ for the BSSD decoupled clock model take the form: 


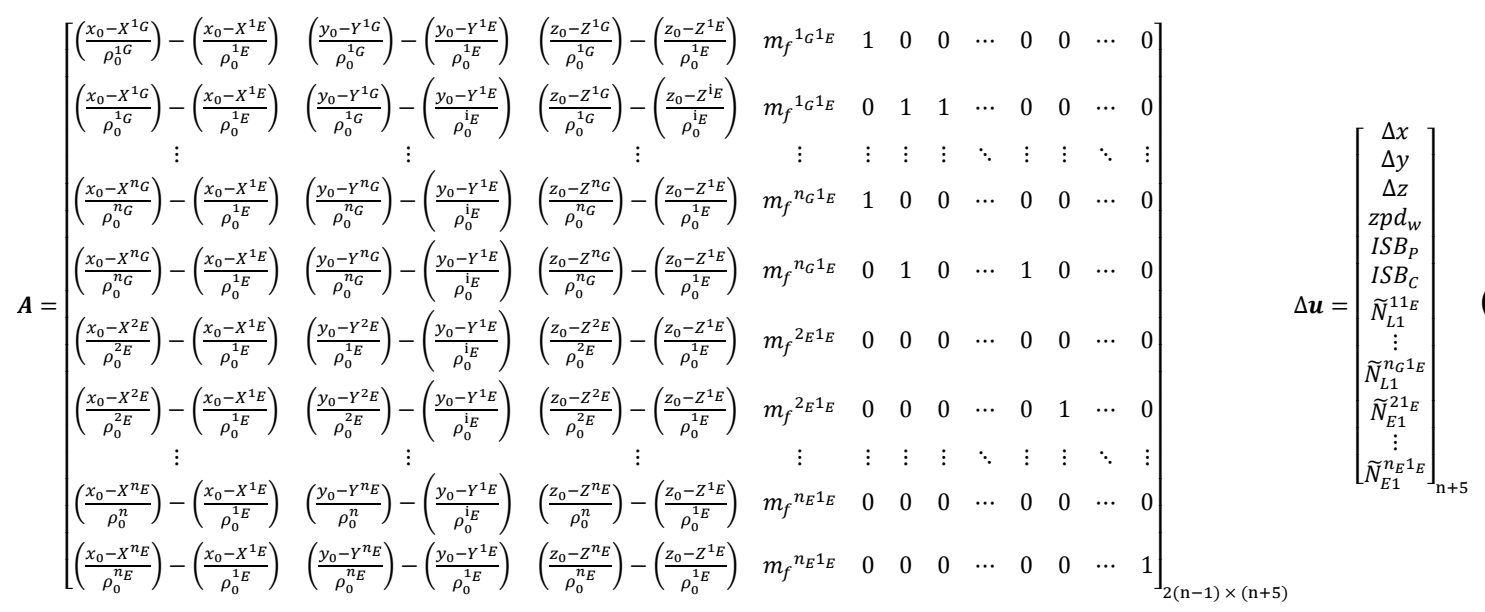

where $l_{E}$ refers to the Galileo reference satellite. For the per-constellation BSSD decoupled clock model, the design matrix $\boldsymbol{A}$ and the vector of corrections $\boldsymbol{\Delta} \boldsymbol{u}$ will take the same form as those of the traditional BSSD GPS/Galileo PPP model. For the BSSD semi-decoupled clock GPS/Galileo PPP model, the design matrix $\boldsymbol{A}$ and the vector of corrections to the unknown parameters $\Delta \mathrm{u}$ will be the same as those of the BSSD decoupled clock model.

\subsection{RESULTS AND DISCUSSION}

To verify the introduced GPS/Galileo PPP models, GPS/Galileo measurements at six welldistributed stations (Figure 6) were selected from the IGS tracking network [23]. Those stations are occupied by GNSS receivers, which are capable of simultaneously tracking the GPS/Galileo constellations. The positioning results for station DLF1 are presented below. Similar results are obtained from the other stations. However, a summary of the convergence times and precision are presented below for all stations. Natural Resources Canada (NRCan) GPSPace PPP software was modified to enable a GPS/Galileo PPP solution as described above. The sampling interval for all data sets is $30 \mathrm{~s}$ of 5 April 2013, while the time span used in the analysis is one hour, which is selected to ensure that the four Galileo satellites are visible at each station. 


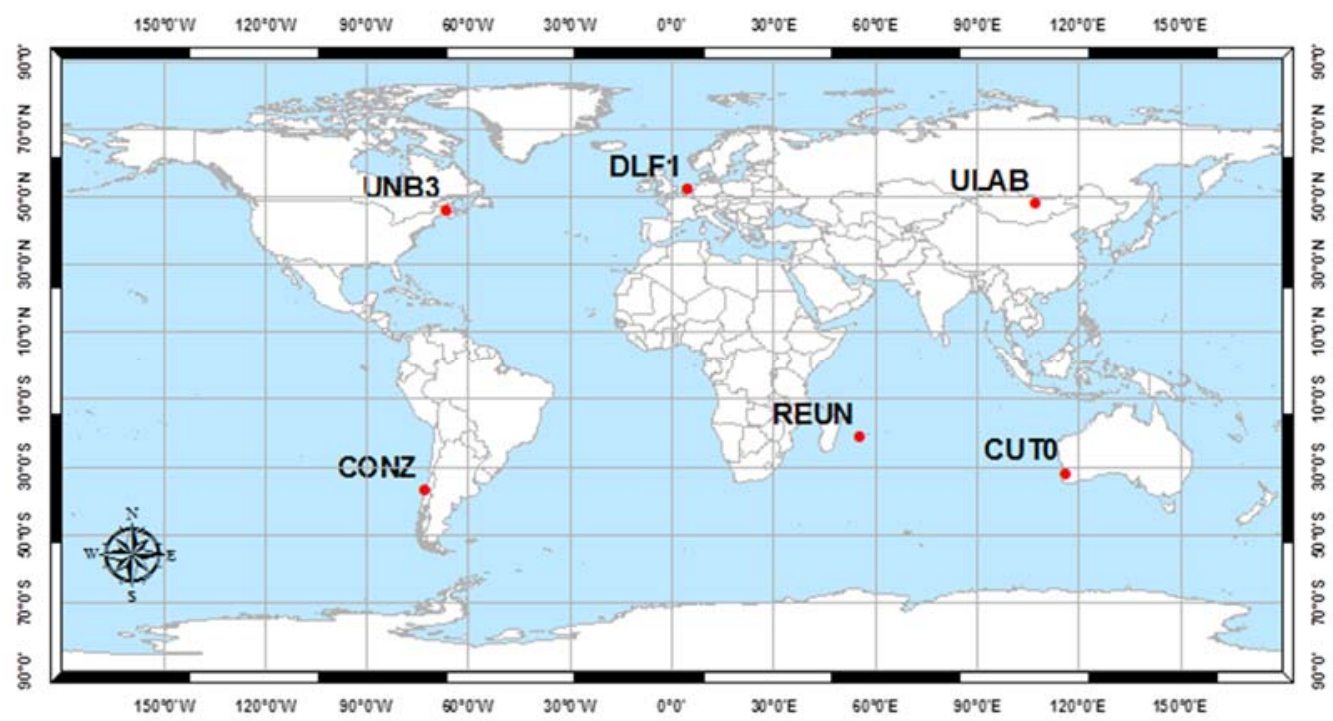

Figure 5. 6 Analysis stations

Figures 5.7 and 5.8 show the positioning results and the estimated ambiguity parameters of the traditional PPP model using GPS/Galileo observations.

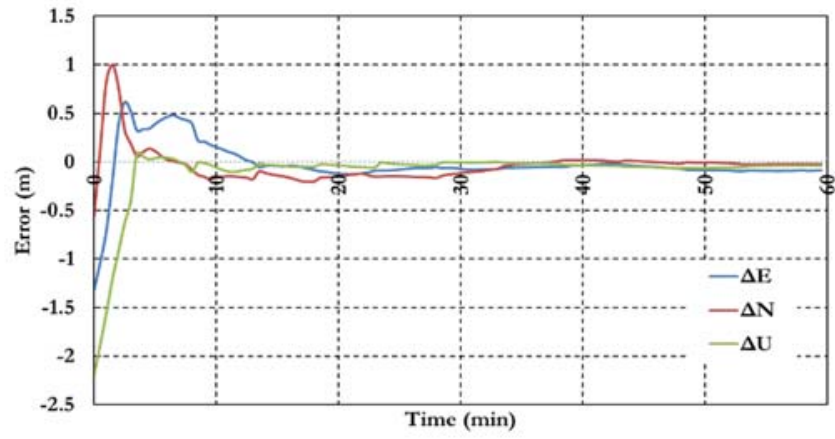

Figure 5. 7 Positioning results of the traditional GPS/Galileo PPP model

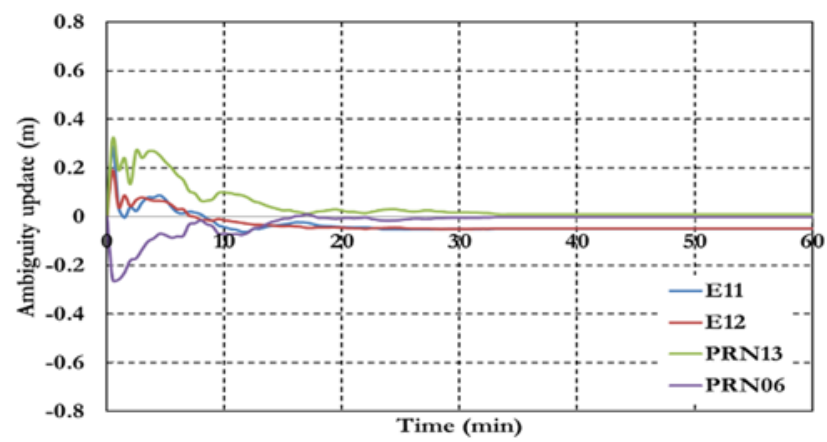

Figure 5. 8 Ambiguity parameters the traditional GPS/Galileo PPP model 
As shown in Figure 5.7, the positioning results of the combined GPS/Galileo traditional PPP model have a convergence time of $15 \mathrm{~min}$ to reach decimeter-level precision. The ambiguity parameters results in Figure 5.8 shows that the un-calibrated hardware delayed that lumped to the ambiguity parameter affects the ambiguity parameters convergence. Figures 5.9-5.11 show the results of the GPS decoupled clock model.

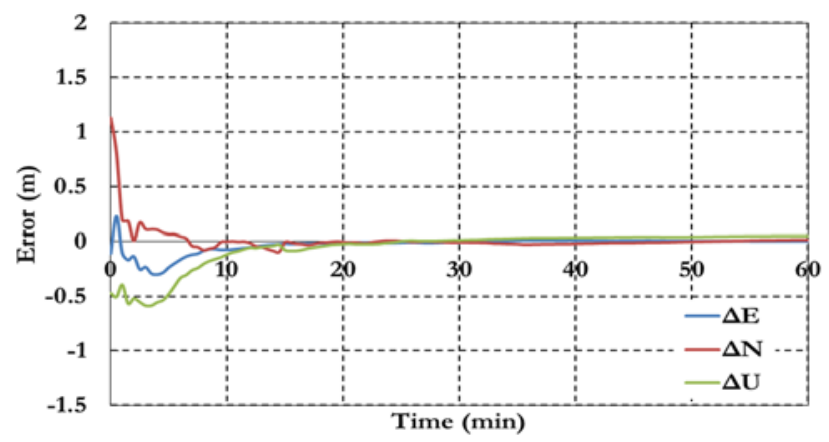

Figure 5. 9 Positioning results of the GPS decoupled clock model

Figure 5.9 shows the positioning results of the decoupled clock model. The results show a decimeter level of precision with about $15 \mathrm{~min}$. Generally the precision of the decoupled clock model positioning results are about $25 \%$ more than the traditional PPP model. Figure 10 shows the receiver clock errors for both pseudorange (CLK_P) and carrier phase (CLK_C) observation.

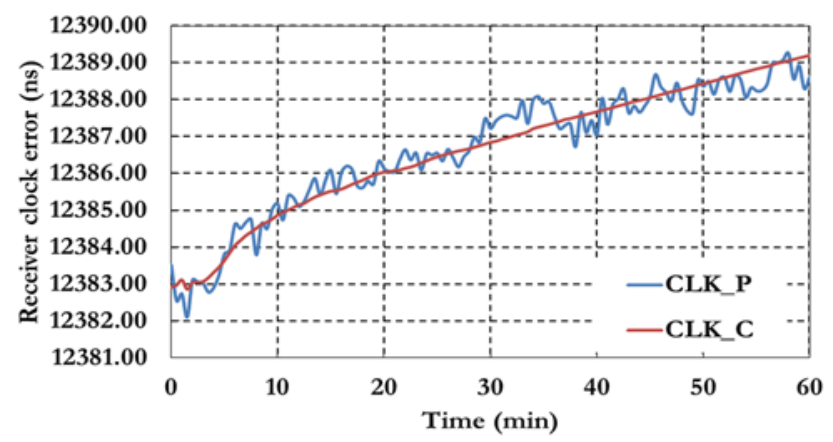

Figure 5. 10 Receiver clock errors of the GPS decoupled clock model

Figure 5.11 shows the results of the ambiguity parameters of the GPS decoupled clock model. 


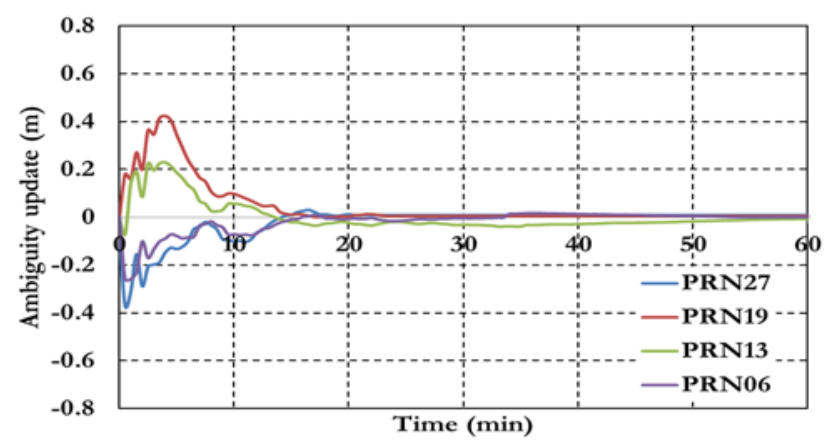

Figure 5. 11 Ambiguity parameters of the GPS decoupled clock model

Figures 5.12-5.15 show the results of the semi-decoupled clock GPS/Galileo PPP model. The positioning results in Figure 5.16 show that the semi-decoupled clock GPS/Galileo PPP model has a decimeter level of precision with about $15 \mathrm{~min}$. In addition, the positioning precision of the semi-decoupled clock GPS/Galileo PPP model are improved by about $25 \%$ comparing to the traditional GPS/Galileo PPP model.

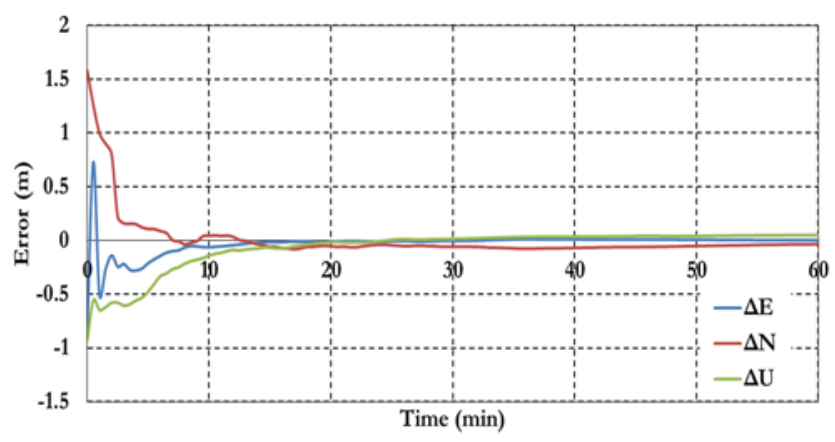

Figure 5. 12 Positioning results of the semi-decoupled clock PPP model

Figure 5.13 shows the receiver clock errors for both pseudorange (CLK_P) and carrier phase (CLK_C) observation. 


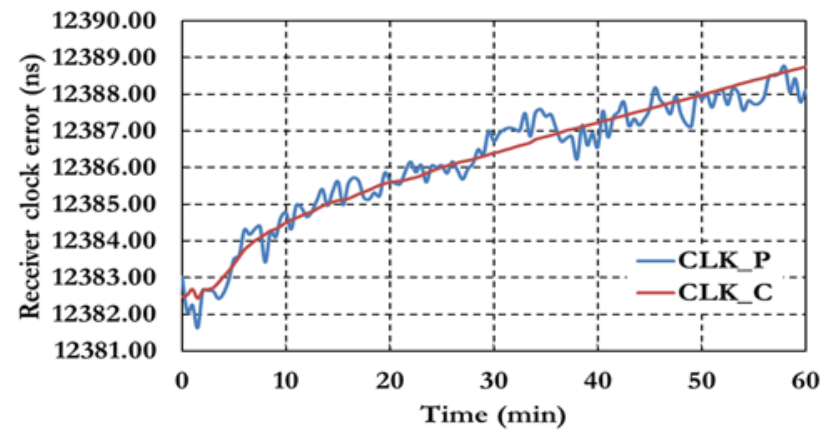

Figure 5. 13 Receiver clock errors of the semi-decoupled clock GPS/Galileo PPP model

Figure 5.14 shows the ambiguity parameters results of the semi-decoupled clock GPS/Galileo PPP model.

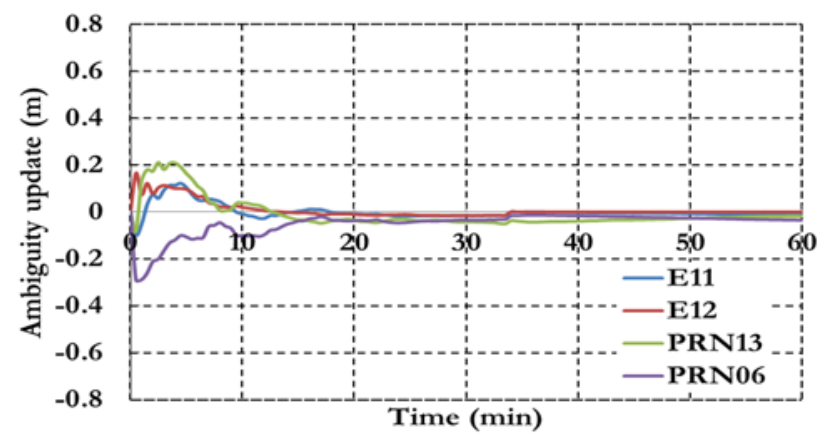

Figure 5. 14 Ambiguity parameters of the semi-decoupled clock GPS/Galileo PPP model

As shown in Figure 5.14 the ambiguity parameters results show a similar convergence time to the positioning results. Figure 5.15 shows the results of the inter-system bias parameters for both pseudorange (ISB_P) and carrier phase (ISB_C) observations.

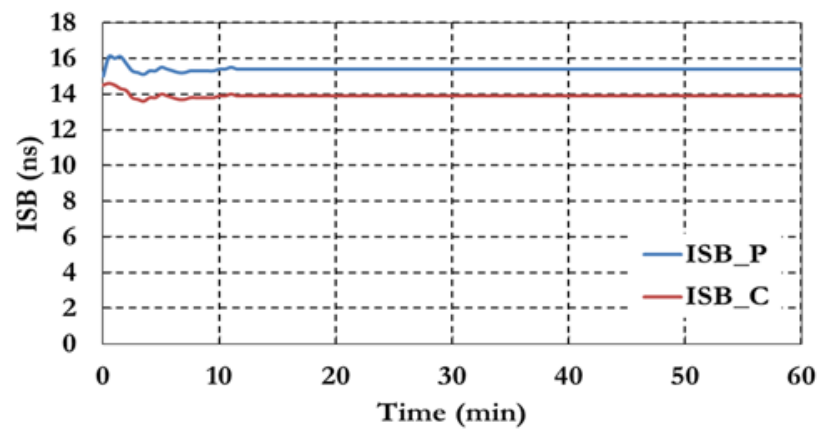

Figure 5. 15 Inter-system bias of the semi-decoupled clock GPS/Galileo PPP model 
Figures 5.16 and 5.17 show the BSSD PPP tight combination model positioning results and the estimated ambiguity parameters.

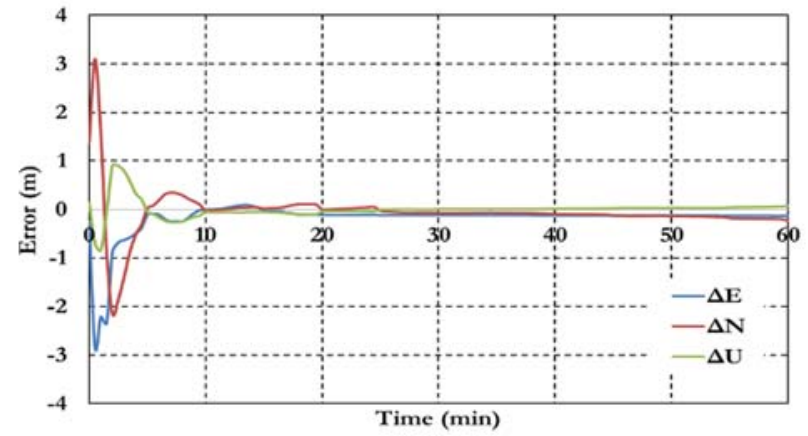

(a)

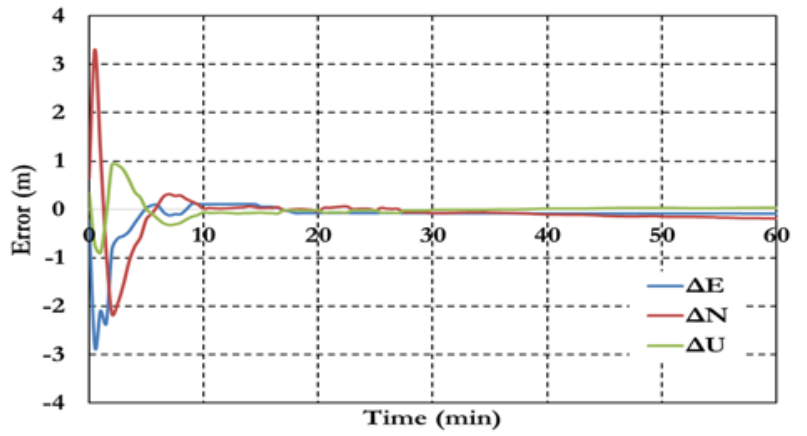

(b)

Figure 5. 16 Positioning results of the BSSD PPP tight combination model using (a) GPS reference satellite; and (b) Galileo reference satellite

As shown in Figure 5.16, the positioning results of the BSSD tight combination model have convergence time of $10 \mathrm{~min}$ and decimeter level of precision.

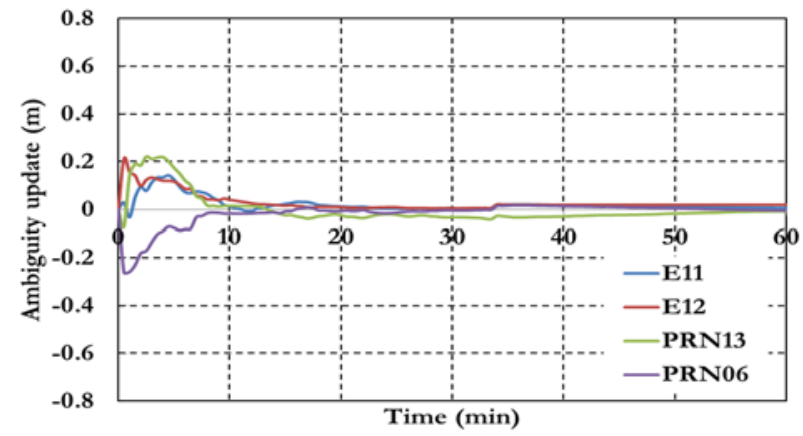

(a)

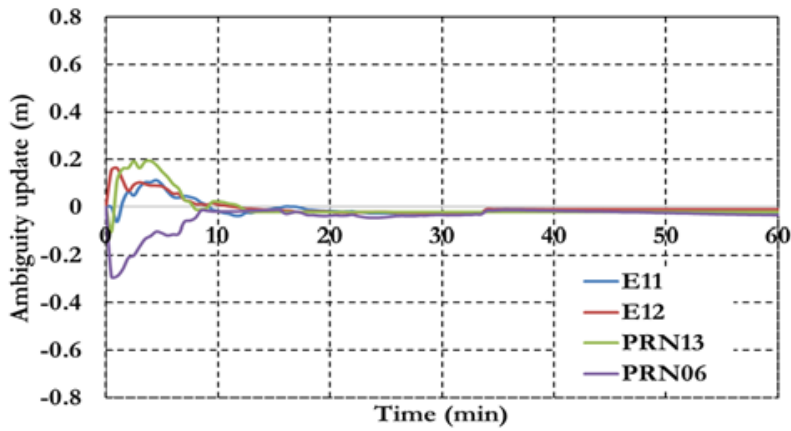

(b)

Figure 5. 17 Ambiguity parameters the BSSD PPP tight combination model using (a) GPS reference satellite; and (b) Galileo reference satellite

As shown in Figure 5.17, the results convergence of the ambiguity parameters are affected by the lumped DCB. Figures 5.18 and 5.19 show the results of the BSSD PPP loose combination model for both positioning results and ambiguity parameters. 


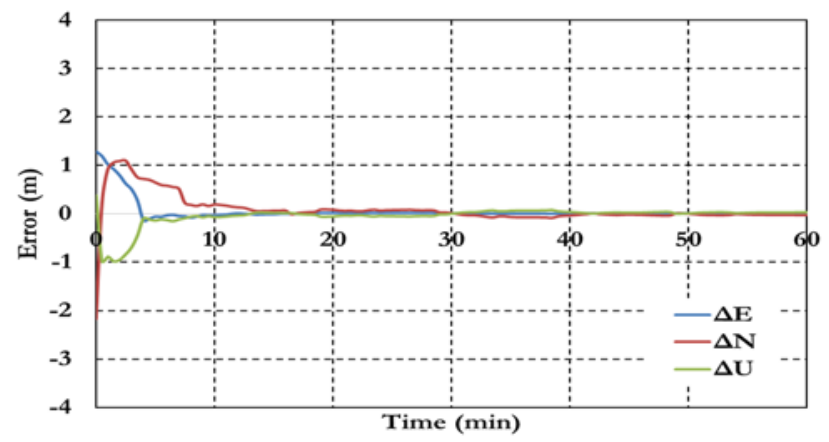

Figure 5. 18 Positioning results of the BSSD PPP loose combination model

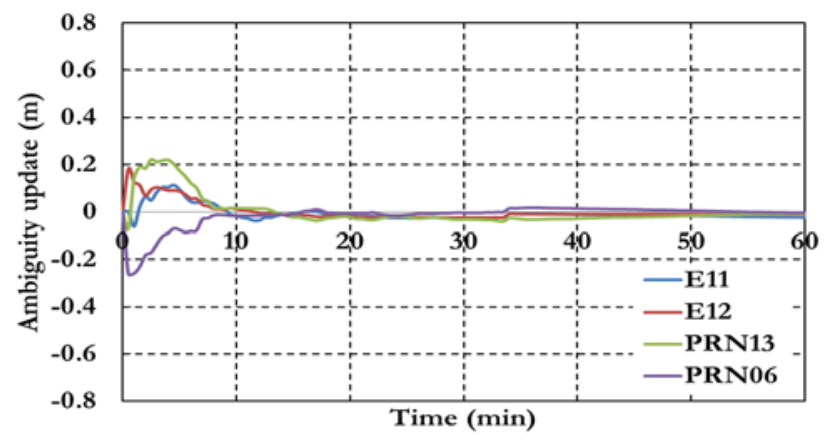

Figure 5. 19 Ambiguity parameters the BSSD PPP loose combination model

As shown in Figure 5.18, the positioning results show decimeter-level precision with about 11 min convergence time. Figure 5.19 shows that the ambiguity parameters of the BSSD loose combination model are affected by the lumped DCB. Figures 5.20 and 5.21 show the positioning results and the estimated ambiguity parameters for the BSSD semi-decoupled GPS/Galileo PPP model, when a tight combination is used. As can be seen, the PPP solution convergences to a decimeter-level precision after about $10 \mathrm{~min}$. 


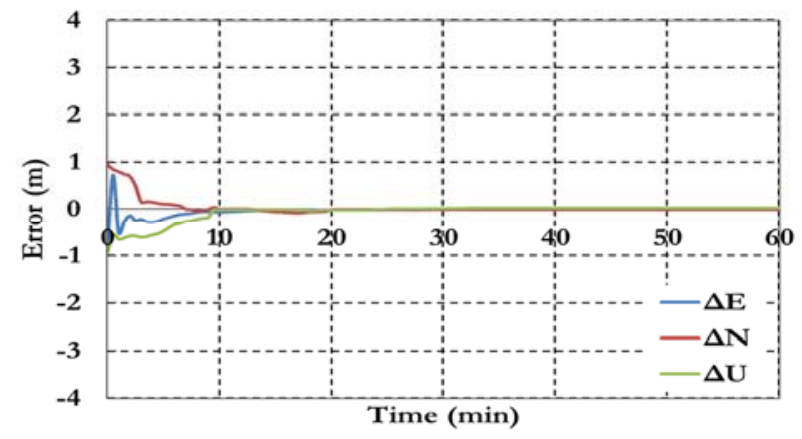

(a)

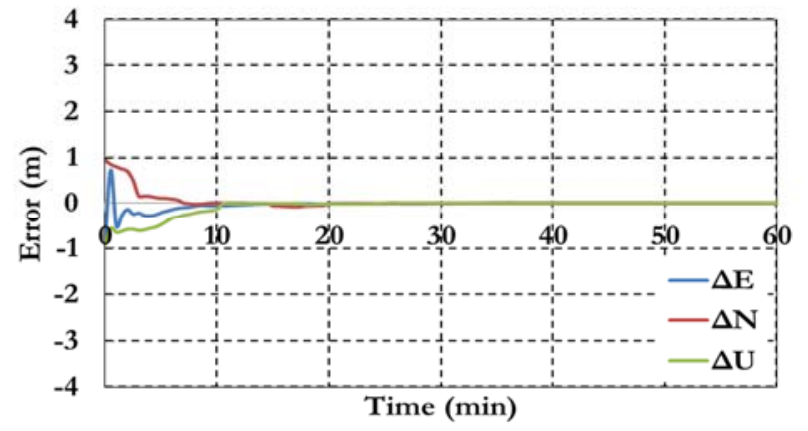

(b)

Figure 5. 20 Positioning results for BSSD semi-decoupled GPS/Galileo PPP model. (a) GPS reference satellite; and (b) Galileo reference satellite

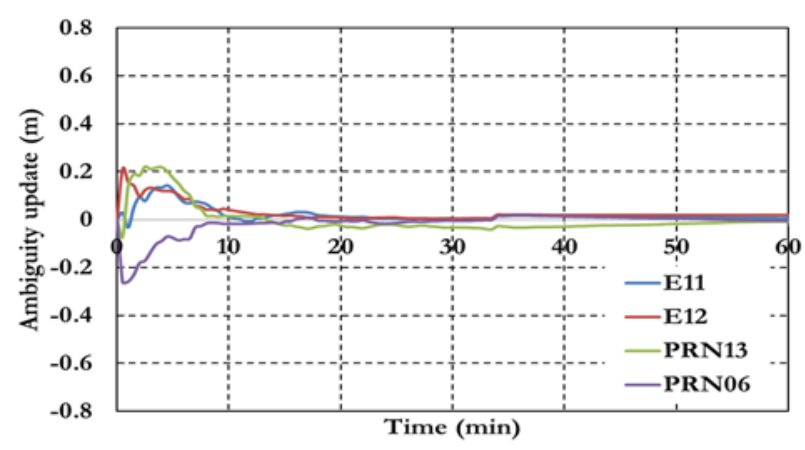

(a)

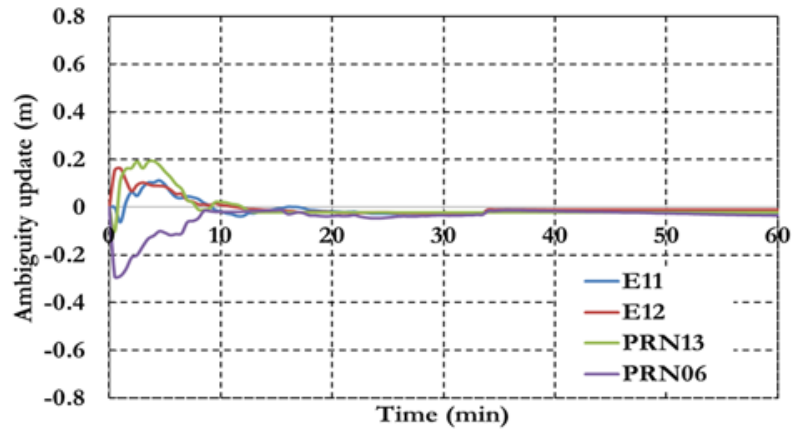

(b)

Figure 5.21 Ambiguity parameters the semi-decoupled GPS/Galileo PPP model (a) GPS reference satellite; and (b) Galileo reference satellite

Figures 5.22 and 5.23 show the results of the semi-decoupled per-constellation GPS/Galileo BSSD PPP model for both of the positioning and ambiguity parameters. As can be seen, the positioning results show decimeter-level precision with about 11 min convergence time. 


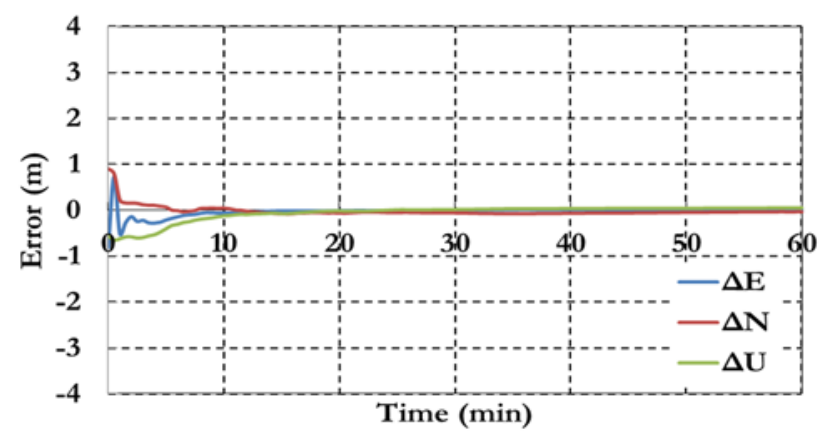

Figure 5. 22 Positioning results of the semi-decoupled per-constellation GPS/Galileo BSSD PPP model

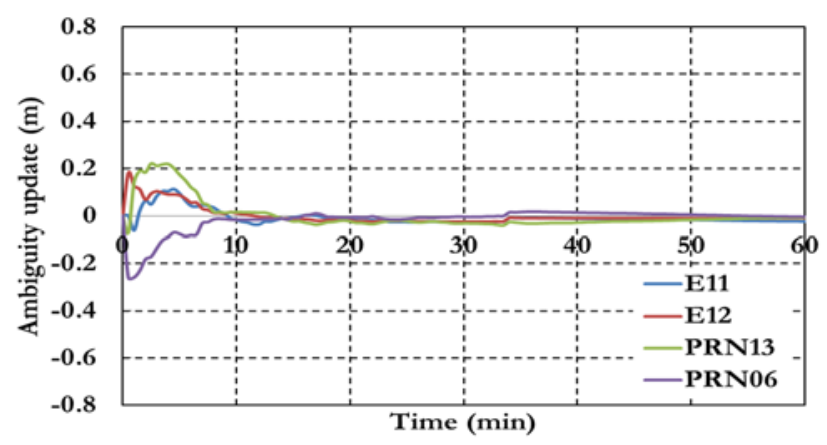

Figure 5. 23 Ambiguity parameters the semi-decoupled per-constellation GPS/Galileo BSSD PPP model

As shown in Figure 5.23, the ambiguity parameters results for both GPS and Galileo satellites are affected by the lumped DCB. Figure 5.24 summarizes the convergence times for all analysis cases, which confirm the PPP solution consistency at all stations.

To further assess the performance of the various PPP models, the solution output is sampled every $10 \mathrm{~min}$ and the standard deviation of the computed station coordinates is calculated for each sample. Figure 5.25 shows the position standard deviations in the East, North, and Up directions, respectively. Examining the standard deviations after $10 \mathrm{~min}$, it can be seen that the semi-decoupled clock GPS/Galileo PPP model improves the precision of the estimated parameters by about $25 \%$ in comparison with the un-differenced GPS-only model. As the number of epochs, and consequently the number of measurements, increases the performance of 
the various models tends to be comparable. An exception, however, is the loose combination model, which is found superior to all other PPP models.

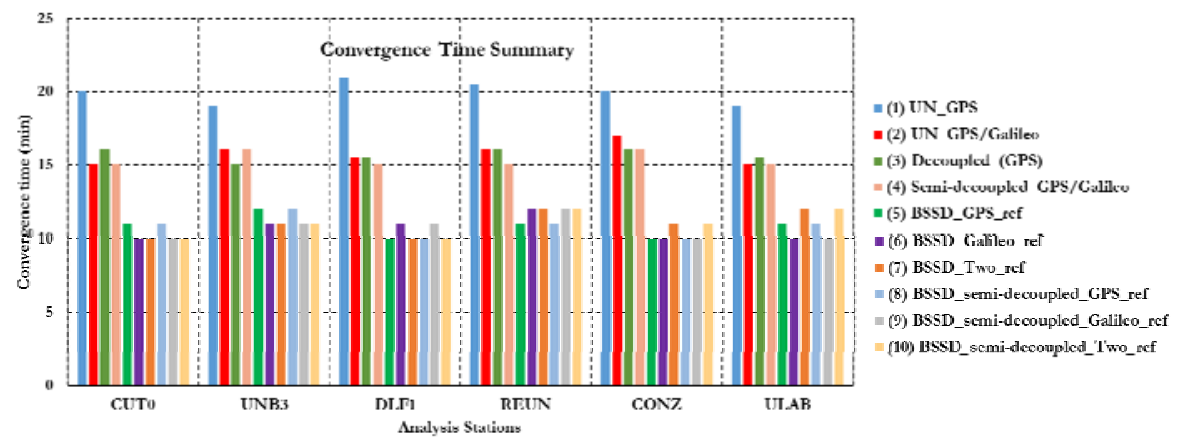

Figure 5. 24 Summary of convergence times of all stations and analysis cases (1) Un-differenced GPS model; (2) Un-differenced GPS/Galileo model; (3) Decoupled clock model using GPS observations only; (4) Semi-decoupled clock GPS/Galileo PPP model; (5) BSSD model with a GPS satellite as a reference; (6) BSSD model with a Galileo satellite as a reference; (7) BSSD model with both a GPS and a Galileo satellite as reference satellites; (8) BSSD semi-decoupled clock GPS/Galileo model with a GPS satellite as a reference; (9) BSSD semi-decoupled clock GPS/Galileo model with a Galileo satellite as a reference; (10) BSSD semi-decoupled clock GPS/Galileo model with both a GPS and a Galileo satellite as reference satellites
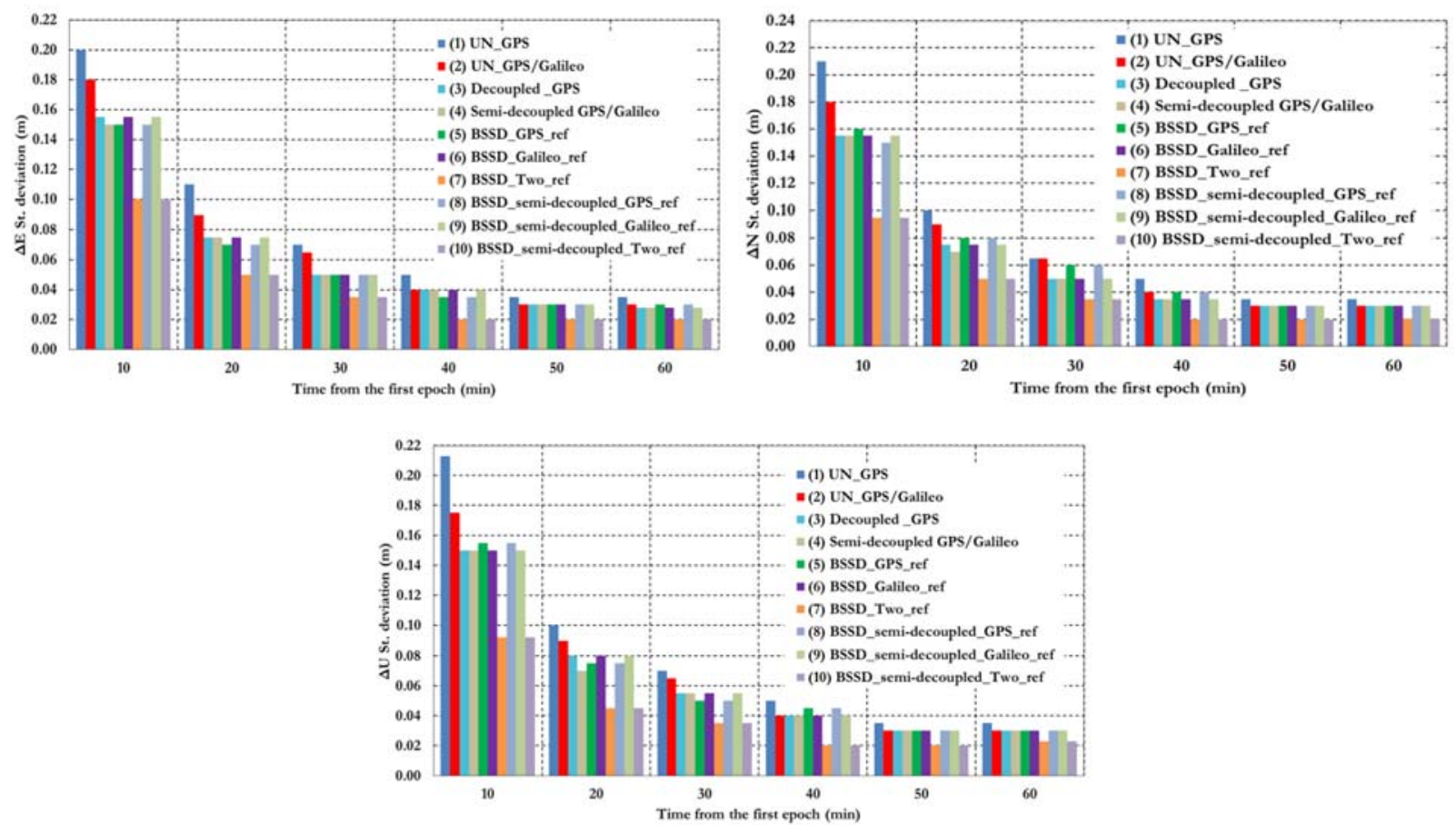
Figure 5. 25 Summary of positioning standard deviations in East, North, and Up directions of all stations and analysis cases. (1) Un-differenced GPS model; (2) Un-differenced GPS/Galileo model; (3) Decoupled clock model using GPS observations only; (4) semi-decouple clock GPS/Galileo PPP model; (5) BSSD model with a GPS satellite as a reference; (6) BSSD model with a Galileo satellite as a reference; (7) BSSD model with both a GPS and a Galileo satellite as reference satellites; (8) BSSD semi-decoupled clock GPS/Galileo model with a GPS satellite as a reference; (9) BSSD semi-decoupled clock GPS/Galileo model with a Galileo satellite as a reference; (10) BSSD semi-decoupled clock GPS/Galileo model with both a GPS and a Galileo satellite as reference satellites

\subsection{CONCLUSIONS}

This paper examined the performance of several PPP models, including the traditional un-differenced model, the decoupled clock model, the semi-decoupled clock model, and BSSD model. It has been shown that the traditional un-differenced GPS/Galileo PPP model, the GPS decoupled clock model, and the semi-decoupled clock GPS/Galileo PPP model improve the convergence time by about $25 \%$ in comparison with the traditional un-differenced GPS-only model. In addition, the semi-decoupled GPS/Galileo PPP model improves the solution precision by about $25 \%$ compared to the traditional un-differenced GPS/Galileo PPP model. Moreover, the BSSD GPS/Galileo PPP model improves the solution convergence time by about $50 \%$, in comparison with the un-differenced GPS PPP model, regardless of the type of BSSD combination used. As well, the BSSD GPS/Galileo model improves the precision of the estimated parameters by about $50 \%$ and $25 \%$ when the loose and the tight combinations are used, respectively, in comparison with the traditional un-differenced GPS-only model. Comparable results are obtained through the tight combination when either a GPS or a Galileo satellite is selected as a reference. 


\section{CHAPTER 6}

\section{CONCLUSIONS AND RECOMMENDATIONS}

This chapter presents the conclusions of this research. In addition, future recommendations are presented in this chapter for future research.

\subsection{CONCLUSIONS}

New stochastic models for the Galileo E1 and E5a signals have been developed in this research. Three functions have been considered, namely exponential, polynomial and rational. It has been found that the exponential function gives the best fit, based on a regression analysis. To test the newly developed stochastic model, GPS/Galileo PPP solutions have been obtained with both of the traditional empirical sine function and the newly developed stochastic models implemented. It has been shown that that a sub-decimeter positioning accuracy is attainable when the developed stochastic model is used. In addition, the convergence time has been improved by about $30 \%$, in comparison with the PPP solution obtained with the empirical sine function implemented.

A single frequency PPP model has been developed, which combines GPS and Galileo observations in the BSSD mode. Two scenarios have been considered when forming the BSSD linear combination, namely a tight and a loose combination. It has been shown that a subdecimeter level positioning accuracy can be obtained with both of the un-differenced and BSSD single-frequency GPS/Galileo PPP models. However, the PPP solution of the un-differenced model takes about 100 minutes to converge to a decimeter level positioning accuracy. In addition, the convergence time of the single-frequency GPS/Galileo PPP solution is improved by $35 \%$ and $15 \%$ when BSSD with tight and loose combinations are used, respectively. The 
moderate improvement in the solution convergence time obtained with the loose combination is likely attributed to its relatively weaker adjustment model in comparison with the tight combination. The ISB is obtained as a by-product of the GPS/Galileo PPP solution for various days and receiver types. Almost identical results have been obtained with both of the undifferenced and BSSD (tight combination) modes. It has been found that the values of the ISB are largely stable over the observation time spans. However, differences of up to $3 \mathrm{~m}$ have been observed, which suggest that the ISB is receiver/firmware dependent.

A dual frequency PPP model, which combines GPS L1/L2 and /Galileo E1/E5a observables has also been developed. It has been shown that the un-differenced PPP positioning results of the GPS-only and GPS/Galileo are at the sub-decimeter level accuracy. However, the convergence time of the combined GPS/Galileo PPP has improved by about $25 \%$ in comparison with the GPS-only PPP. The use of BSSD linear combination improved the convergence time of the GPS/Galileo PPP solution by about 50\%, in comparison with the un-differenced GPS-only PPP model, regardless of the type of BSSD combination used. In addition, the BSSD model improved the precision of the estimated parameters by about $50 \%$ and $25 \%$, in comparison with the undifferenced GPS-only model, when the loose and the tight combinations are used, respectively. As the number of epochs increases, the performance of the various models tends to be comparable. An exception, however, is the loose combination model, which is found superior to all other PPP models. Almost identical results are obtained through the tight combination when either a GPS or a Galileo satellite is selected as a reference. Similar to the single frequency PPP model, the ISB is obtained as a by-product of the GPS/Galileo PPP solution for various days and receiver types. It has been shown that ISB is essentially constant over the observation time span with a magnitude ranging from 30 to 60 nanoseconds, depending on the GNSS receiver type.

The performance of several PPP models, which combine dual-frequency GPS/Galileo observations in the un-differenced and BSSD modes, has been examined. These models include the traditional un-differenced model, the decoupled clock model, the semi-decoupled clock model, and the BSSD model. The IGS-MGEX network products have been considered to correct for the satellite differential code biases, the orbital and satellite clock errors. It has been shown that the traditional un-differenced GPS/Galileo PPP model, the GPS decoupled clock model, and 
semi-decoupled clock GPS/Galileo PPP model improve the convergence time by about $25 \%$ in comparison with the un-differenced GPS-only model. In addition, the semi-decoupled GPS/Galileo PPP model improves the solution precision by about $25 \%$ compared to the traditional un-differenced GPS/Galileo PPP model. Moreover, the BSSD GPS/Galileo PPP model improves the solution convergence time by about $50 \%$, in comparison with the un-

differenced GPS PPP model, regardless of the type of BSSD combination used. As well, the BSSD model improves the precision of the estimated parameters by about $50 \%$ and $25 \%$ when the loose and the tight combinations are used, respectively, in comparison with the undifferenced GPS-only model. Comparable results are obtained through the tight combination when either a GPS or a Galileo satellite is selected as a reference.

\subsection{RECOMMENDATIONS}

To further improve the findings of this dissertation, further research is needed in the following areas:

1. To improve the PPP solution precision and convergence time, additional GNSS precise products are required, including the differential carrier-phase bias, and the decoupled clock products for Galileo systems.

2. A new model for instantaneous PPP ambiguity resolution is needed, which leads to realtime PPP at the centimeter-level.

3. The developed GPS/Galileo PPP models should be extended to include multiconstellation GNSS PPP.

4. The data sets used in this research are static. The developed GPS/Galileo PPP models should be tested using kinematic data sets.

5. Precise real-time orbit and clock corrections, as well as differential code and carrierphase biases, for all four constellations are needed to test the PPP model in real time. 


\section{REFERENCES}

Abd El-Rahman, M. A., and El-Rabbany, A. (2013). Performance evaluation of USTEC product for single-frequency precise point positioning. Geomatica, 67(4), 253-257. doi: 10.5623/CIG2013-049.

Afifi, A. and El-Rabbany, A. (2013). Stochastic modeling of Galileo E1 and E5a signals. International Journal of Engineering and Innovative Technology (IJEIT), 3(6), pp. 188192.

Afifi, A. and El-Rabbany, A. (2015). An innovative dual frequency PPP model for combined GPS/Galileo observations. Journal of Applied Geodesy, 9(1). 27-34, doi:10.1515/jag2014-0009.

Afifi, A., and El-Rabbany, A. (2014). Improved model for precise point positioning with dual frequency GPS/Galileo observables. The International Archives of the Photogrammetry, Remote Sensing and Spatial Information Sciences, Volume XL-2, 2014 ISPRS Technical Commission II Symposium, 6 - 8 October 2014, Toronto, Canada.

BeiDou, (2015). BeiDou navigation satellite system, Retrieved June 28, 2015 (http://en.beidou.gov.cn/).

Blewitt G (1989) Carrier phase ambiguity resolution for the global positioning system applied to geodetic baselines up to 2,000 km. J Geophys Res 94(B8):10187-10203. doi:10.1029/JB094iB08p10187.

Boehm, J. and Schuh, H. (2004). Vienna mapping functions in VLBI analyses. Geophysical Research Letters 31(1): L01603 01601-01604. doi: 10.1029/2003g1018984.

Bos, M.S, and Scherneck, H.-G. (2011). Ocean tide loading provider. Retrieved December 1, 2014, from http://holt.oso.chalmers.se/loading/ 
Chen, K., and Gao, Y. (2005). Real-time precise point positioning using single frequency data. Paper presented at the 18th international technical meeting of the satellite division of The Institute of Navigation, ION GNSS 2005, September 13, 2005 - September 16, 2005 , Long Beach, CA, United states.

Collins P, Lahaye F, Héroux P, and Bisnath S (2008) Precise point positioning with ambiguity resolution using the decoupled clock model. In: Proceedings of ION GNSS 21st international technical meeting of the satellite division, Savannah, US, pp 1315-1322

Collins, P., S. Bisnath, F. Lahaye, and P. Héroux (2010). Undifferenced GPS Ambiguity Resolution Using the Decoupled Clock Model and Ambiguity Datum Fixing. NAVIGATION, Vol. 57, No. 2, pp. 123-135.

Colombo, O.L., A.W. Sutter, and Evans, A.G. (2004). Evaluation of precise, kinematic GPS point positioning. ION GNSS 17th international technical meeting of the satellite division, 21-24 Sept. 2004, Long Beach, CA.

Dong D, and Bock Y (1989) Global positioning system network analysis with phase ambiguity resolution applied to crustal deformation studies in California. J Geophys Res 94(B4):3949-3966. doi: 10.1029/JB094iB04p03949.

Dow, J.M., Neilan, R. E., and Rizos, C. (2009). The international GNSS service in a changing landscape of global navigation satellite systems. Journal of Geodesy 83(3-4), 191-198, doi: 10.1007/s00190-008-0300-3.

Draper, N. R. (2002). Applied regression analysis: bibliography update 2000-2001. Communications in Statistics - Theory and Methods, 31(11), 2051-2075. doi: $10.1081 /$ sta-120015017 
El-Rabbany, A. (1994), The effect of physical correlations on the ambiguity resolution and accuracy estimation in gps differential positioning, Department of Geodesy and Geomatics Engineering, University of New Brunswick, Doctoral dissertation, http://www2.unb.ca/gge/Pubs/TR170.pdf

El-Rabbany, A. (2006). Introduction to GPS: The global positioning system. Artech House Publishing.

Elsobeiey, M. and A. El-Rabbany (2010). On stochastic modeling of the modernized global positioning system (GPS) L2C signal. Journal of Measurement Science and Technology, Volume 21, Number 5, pp. 1-6.

Elsobeiey, M. and A. El-Rabbany (2013). An efficient precise point positioning model for near real-time applications. Institute of Navigation International Technical Meeting 2013, ITM 2013, January 28, 2013 - January 30, 2013, San Diego, CA, United states, Institute of Navigation.

Elsobeiey, M. and A. El-Rabbany (2014). Efficient between-satellite single-difference precise point positioning model. Journal of surveying engineering 140(2): 04014007 (04014007 pp.). doi: 10.1061/(asce)su.1943-5428.0000125.

ESA (2015). European Space Agency, Retrieved June 28, 2015, from (http://www.esa.int/ESA).

European Space Agency, ESA (2013). Galileo and GPS synchronise watches: new time offset helps working together. Retrieved May 18, 2013, from (http://www.esa.int/Our_Activities/Navigation/Galileo_and_GPS_synchronise_watches_ new_time_offset_helps_working_together).

Gabor MJ, Nerem RS (1999) GPS carrier phase ambiguity resolution using satellite-satellite single difference. In: Proceedings of ION GNSS 12th international technical meeting of the satellite division, Nashville, US, pp 1569-1578. 
Gao, Y. and X. Shen (2001), "Improving ambiguity convergence in carrier phase-based precise point point positioning", ION GPS 2001, Salt Lake City, UT, 11-14 September, pp. 15321539.

Gao, Y. and X. Shen (2002), "Ambiguity pseudo-fixing in precise single point positioning". ION 58th Annual Meeting/CIGTF 21st Guidance Test Symposium, 24-26 June 2002, Albuquerque, NM, PP 163-168.

Ge, M., Gendt, G., Rothacher, M., Shi, C., and Liu, J. (2008). Resolution of GPS carrier-phase ambiguities in precise point positioning (PPP) with daily observations. Journal of Geodesy, 82(7), 401. doi: 10.1007/s00190-007-0208-3.

Heroux, P., and Kouba, J. (2001). GPS precise point positioning using IGS orbit products. Physics and Chemistry of the Earth, Part A: Solid Earth and Geodesy, 26(6-8), 573-578. doi: 10.1016/s1464-1895(01)00103-x.

Hofmann-Wellenhof, B., H. Lichtenegger, and E. Wasle (2008). GNSS global navigation satellite systems; GPS, Glonass, Galileo \& more. Springer Wien New York. 501pp.

Hopfield, H. S. (1972). Tropospheric refraction effects on satellite range measurements. APL Technical Digest, 11(4), 11-19.

IAC (2015). Fedral space agency,the Information-analytical centre, Retrieved June 28, 2015, from (https://glonass-iac.ru/en/)

IERS (2010). International earth rotation and reference system services conventions. IERS Technical Note 36, available at http://www.iers.org/IERS/EN/Publications/TechnicalNotes/tn36.html/. 
International GNSS service (IGS), 2015. Multi-GNSS differential code biases (DCBs), Retrieved February 25, 2015. Available at ftp://cddis.gsfc.nasa.gov/pub/gps/products/mgex/dcb/.

Kaplan, E., and Hegarty, C. (2006). Understanding GPS principles and applications. 2nd edition, Artech House Inc. 683 pp.

King M, Colemar R, and Nguyen LN (2003) Spurious periodic horizontal signals in sub-daily GPS position estimates. J Geod 77(1-2): 15-21.

Klobuchar, J.A. (1991). Ionospheric Effects on GPS. GPS World, Vol. 2, No. 4, April 1991, pp. 48-51.

Kouba, J. (2009). A guide to using International GNSS Service (IGS) products. Available at http://igscb.jpl.nasa.gov/igscb/resource/pubs/UsingIGSProductsVer21.pdf.

Kouba, J. and P. Heroux (2001) Precise point positioning using IGS orbit and clock products. GPS Solutions, 5(2), PP 12-28. doi:10.1007/PL00012883.

Laurichesse D, Mercier F, Berthias JP, Broca P, and Cerri L (2009) Integer ambiguity resolution on undifferenced GPS phase measurements and its application to PPP and satellite precise orbit determination. Journal of The Institute of Navigation, 56(2):135-149.

Leandro, R. F. and M. C. Santos (2007). Stochastic models for GPS positioning: An empirical approach. GPS World 18(2): 50-56.

Leick, A. (1995). Book review: GPS Satellite Surveying. Surveying and Land Information Systems 55(4): 219-219.

Melbourne, W.G. (1985). The case for ranging in GPS based geodetic systems. Proceedings of 1 st international symposium on precise positioning with the global positioning system, 
edited by Clyde Goad, U.S. Department of Commerce, Rockville, Maryland, 15-19 April, pp. 373-386.

Melgard, T., Tegedor, J., de Jong, K., Lapucha, D., and Lachapelle, G. (2013). Interchangeable integration of GPS and Galileo by using a common system clock in PPP. ION GNSS+, September 16-20, 2013, Institute of Navigation, Nashville TN.

Mercier F, and Laurichesse D (2008) Zero-difference ambiguity blocking properties of satellite/receiver widelane biases. In: Proceedings of European navigation conference, Toulouse, France Meyer CD (2000) Matrix analysis and applied linear algebra. Soc Ind Appl Math, Philadelphia.

Montenbruck O., Steigenberger P., Khachikyan R., Weber G., Langley R.B., Mervart L., and Hugentobler U. (2014). IGS-MGEX: preparing the ground for multi-constellation GNSS science, InsideGNSS 9(1):42-49.

Montenbruck, O., Hauschild, A., and Steigenberger, P. (2014). Differential Code Bias Estimation using Multi-GNSS Observations and Global Ionosphere Maps. Navigation, 61(3), 191201. doi: 10.1002/navi.64

Montenbruck, O., Hauschild, A., Hessels, U., Steigenberger, P., and Hugentobler, U. (2009). CONGO first GPS/Giove tracking network for science, research. GPS World, 20(9), 5662.

Montenbruck, O., Steigenberger P., Khachikyan R., Weber G., Langley R.B., Mervart L., and Hugentobler U. (2014). IGS-MGEX: Preparing the ground for multi-constellation GNSS science, InsideGNSS 9(1):42-49.

Odijk, D., and Teunissen, P. J. G. (2013). Characterization of between-receiver GPS-Galileo inter-system biases and their effect on mixed ambiguity resolution. GPS Solutions, 17(4), 521-533. doi: 10.1007/s10291-012-0298-0. 
Paziewski J and Wielgosz P (2014). Assessment of GPS + Galileo and multi-frequency Galileo single-epoch precise positioning with network corrections. GPS Solut, 18(4):571:579, doi $10.1007 / \mathrm{s} 10291-013-0355-3$

Paziewski, J., and Wielgosz, P. (2015). Accounting for Galileo-GPS inter-system biases in precise satellite positioning. J Geod. DOI 10.1007/s00190-014-0763-3.

Schaer, S., W. Gurtner, and J. Feltens. (1998). IONEX: The IONosphere Map EXchange Format Version 1. Proceedings of the IGS AC Workshop, Darmstadt, Germany, 9-11 February.

Shi, J., and Gao, Y. (2014). A comparison of three PPP integer ambiguity resolution methods. GPS Solutions, 18(4), 519-528. doi: 10.1007/s10291-013-0348-2.

Steigenberger P., Hugentobler U., Loyer S., Perosanz F., Prange L., Dach R., Uhlemann M., Gendt G., and Montenbruck O. (2014). Galileo orbit and clock quality of the IGS multiGNSS experiment. Advances in Space Research, doi: 10.1016/j.asr.2014.06.030.

Tegedor, J., Liu, X., de Jong, K., Goode, M., Øvstedal, O., and Vigen, E. (2014), Estimation of Galileo uncalibrated hardware delays for ambiguity-fixed precise point positioning, Proceedings of the 27th international technical meeting of the satellite division of the institute of navigation (ION GNSS+ 2014), Tampa, Florida, September 2014, pp. 2346-2353.

Teunissen PJG, and Kleusberg A (1998) GPS for geodesy. Springer Verlag, Berlin Heidelberg New York. Leick A (1995) GPS satellite surveying. John Wiley and Sons, Inc., New York, N.Y.

Tregoning P, and Watson C (2009) Atmospheric effects and spurious signals in GPS analysis. J Geophys Res 114:B09403. doi:10.1029/2009JB006344. 
Vanicek, P., and Krakiwsky, E. J. (1986). Geodesy: The concepts. 2nd edition, North-Holland, Amsterdam, The Netherlands.

Wu, J. T., S, C. Wu, G.A. Hajj, W.I. Bertiger, and S. M. Lichten (1993). Effects of antenna orientation on GPS carrier phase. Man. Geodetica 18, pp. 91-98.

Wübbena G (1985) Software developments for geodetic positioning with GPS using TI-4100 code and carrier measurements. In: Proceedings of first international symposium on precise positioning with the global positioning system, Rockville, US, pp 403-412.

Zumberge, J.F., M.B. Heflin, D.C. Jefferson, M.M. Watkins and F.H. Webb (1997). Precise point processing for the efficient and robust analysis of GPS data from large networks. Journal of Geophysical Research, Vol. 102, No. B3, pp 5005-5017. 